University of Louisville

ThinkIR: The University of Louisville's Institutional Repository

Electronic Theses and Dissertations

$12-2016$

\title{
Cost of care for hospitalized patients with pulmonary mycobacterial diseases in the United States.
}

Mary Beth Wells Allen

University of Louisville

Follow this and additional works at: https://ir.library.louisville.edu/etd

Part of the Bacterial Infections and Mycoses Commons, and the Other Public Health Commons

\section{Recommended Citation}

Allen, Mary Beth Wells, "Cost of care for hospitalized patients with pulmonary mycobacterial diseases in the United States." (2016). Electronic Theses and Dissertations. Paper 2590.

https://doi.org/10.18297/etd/2590

This Doctoral Dissertation is brought to you for free and open access by ThinkIR: The University of Louisville's Institutional Repository. It has been accepted for inclusion in Electronic Theses and Dissertations by an authorized administrator of ThinkIR: The University of Louisville's Institutional Repository. This title appears here courtesy of the author, who has retained all other copyrights. For more information, please contact thinkir@louisville.edu. 


\title{
COST OF CARE FOR HOSPITALIZED
}

PATIENTS WITH PULMONARY

\section{MYCOBACTERIAL DISEASES IN THE UNITED}

\section{STATES}

\author{
By \\ Mary Elizabeth Wells Allen \\ B.A. University of Kentucky, May 2002 \\ M.B.A. University of Louisville, December 2006
}

\author{
A Dissertation \\ Submitted to the Faculty of the \\ School of Public Health and Information Sciences of the \\ University of Louisville \\ in Partial Fulfillment of the Requirements \\ for the Degree of
}

Doctor of Philosophy in Public Health Science

School of Public Health and Information Sciences

Public Health Management and Systems Sciences

University of Louisville

Louisville, Kentucky

December 2016 
Copyright 2016 by Mary Elizabeth Wells Allen

All right reserved 



\title{
COST OF CARE FOR HOSPITALIZED
}

\section{PATIENTS WITH PULMONARY}

\section{MYCOBACTERIAL DISEASES IN THE UNITED STATES}

\author{
By \\ Mary Elizabeth Wells Allen \\ B.A., University of Kentucky \\ M.B.A., University of Louisville
}

A Dissertation approved on

November 30, 2016

By the following Dissertation Committee:

Susan Olson Allen, PhD (Committee Chair)

Robert Esterhay, MD

Michael Mills, PhD, PhD

Mehdi Mirsaeidi, MD, MPH 


\section{DEDICATION}

This dissertation is dedicated to my children, as they are the reason for everything. I dedicate this work to my daughter, Lydia, my son, Ryan, and my daughter, Ava, who we lost. 


\section{ACKNOWLEDGEMENTS}

I would like to express my gratitude to all of those who supported me through this process. I am especially grateful to my parents. I would give anything for my father, Alvin Wells, to be here with me as I complete this degree. He believed in me more than any other and he went to great lengths to make sure I had access to education and opportunities since I was a child. My mother, Donna Wells, had this dream for me before I was old enough to have dreams. Without her infinite support including being on call for childcare, having the many hours of quiet time I needed to complete this project would not have been possible. I would like to thank my husband, Marty, who has been my inspiration, and the family we have built together will always represent my

greatest achievement. I would like to thank my family, my chosen family, and my incredible friends. Thank you all for supporting me, loving me, sacrificing for me, advising me, and giving me space to work. I would also like to thank the many great mentors I have encountered during my education, as there have been too many to name. I would especially like to thank my committee members, who believed I was worth the investment of their time and energy. They worked hard along with me, challenged me, supported me, and, in the end, truly had my best interest at heart. This achievement belongs to all of us. 


\section{ABSTRACT}

COST OF CARE FOR HOSPITALIZED PATIENTS WITH PULMONARY MYCOBACTERIAL DISEASES IN THE UNITED STATES

Mary Elizabeth Wells Allen

November 30, 2016

Background: Pulmonary mycobacterial diseases describe both tuberculosis (TB) and nontuberculous mycobacteria (NTM). Few data are available measuring the cost burden of mycobacterial diseases on the national level. The purpose of this study was to evaluate the cost burden and measure emerging trends in hospitalization of pulmonary TB and NTM in the US from 2001 through 2012.

Methods: This study was a retrospective community based cost analysis of hospitalized patients with a principal diagnosis of pulmonary mycobacterial diseases from 2001 through 2012. Data for pulmonary TB and NTM were retrieved from the Healthcare Cost and Utilization Project (HCUP), US Department of Health and Human Services. The statistical significance of observed trends of NTM and TB national hospital costs 
was calculated using Poisson log-linear regression.

Results: A total of 20,049 hospital admissions were reported for pulmonary NTM and 69,257 for pulmonary TB in the US from 2001 through 2012. The total associated cost of these admissions was $\$ 903,767,292$ for pulmonary NTM and $\$ 2,078,113,317$ for pulmonary TB. During the study period, the national hospital costs of pulmonary NTM increased at a statistically significant rate in the US over each year $(P=0.001)$. However, no such increase was found for national hospital costs of pulmonary TB.

Conclusion: The national hospital cost of NTM management is increasing. These results emphasize the importance of continued research in pulmonary NTM in order to improve current guidelines in prevention and treatment strategies. 


\section{TABLE OF CONTENTS}

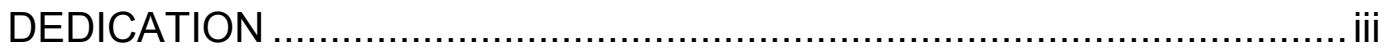

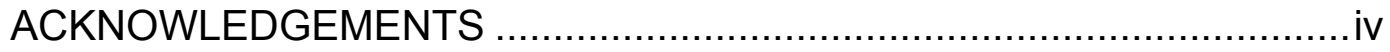

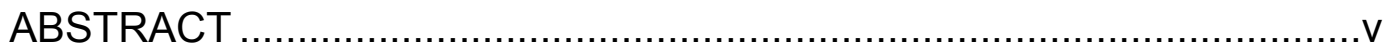

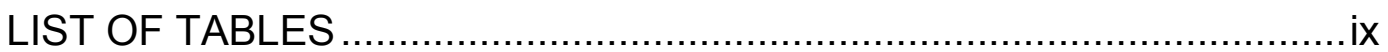

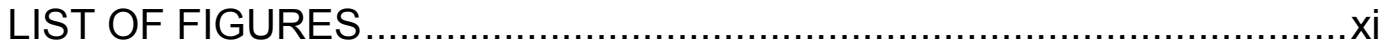

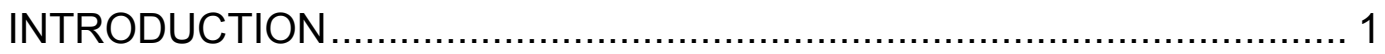

CHAPTER 1: OVERVIEW OF MYCOBACTERIUM DISEASE ................. 3

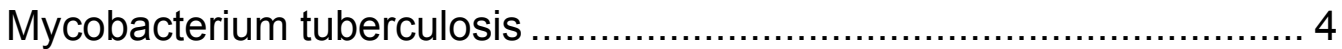

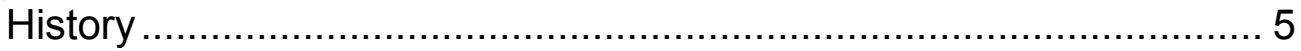

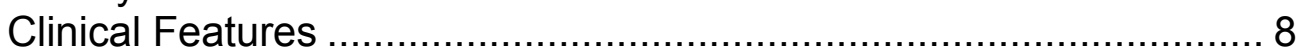

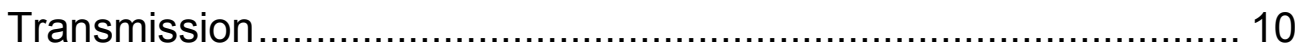

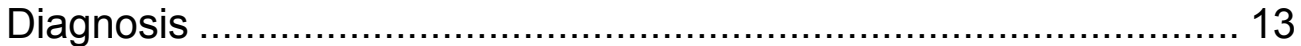

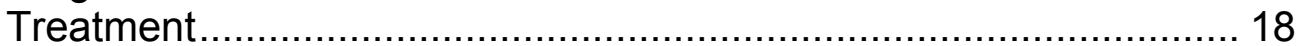

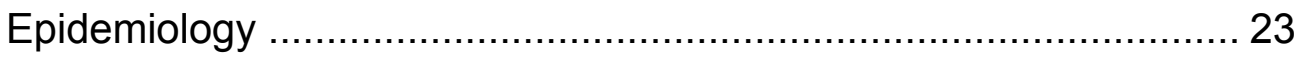

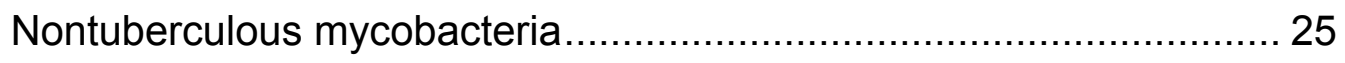

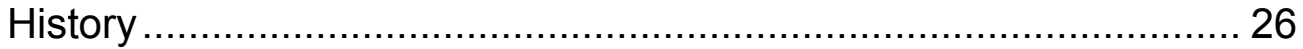

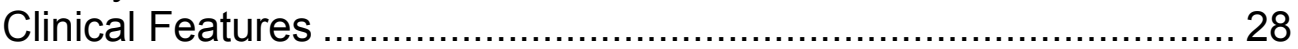

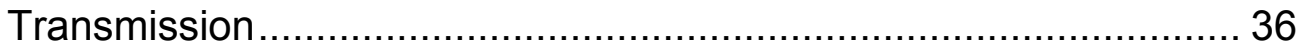

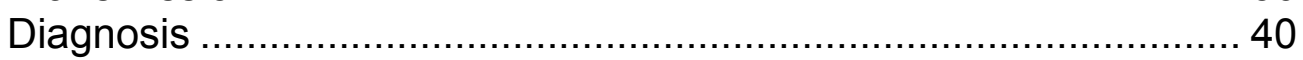

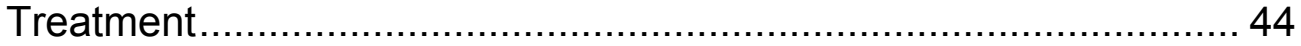

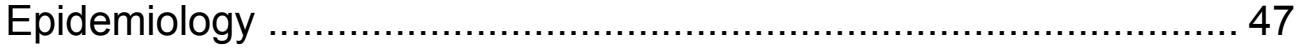

CHAPTER 2: STATEMENT OF THE PROBLEM AND

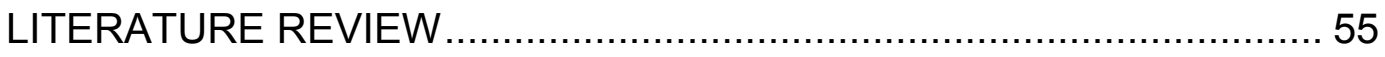

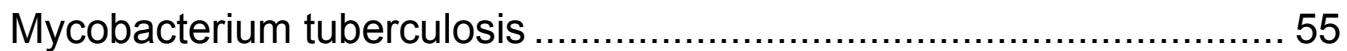


TB in the Underdeveloped World ....................................................56 56

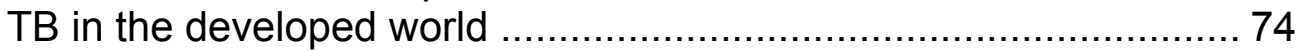

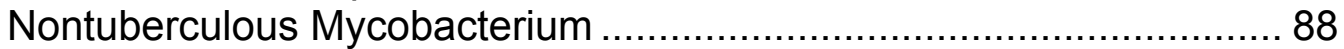

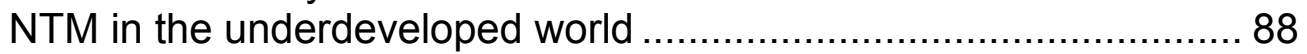

NTM in the developed world .................................................... 94

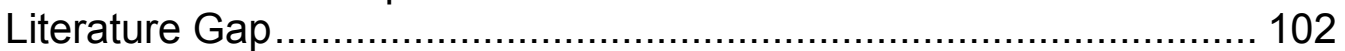

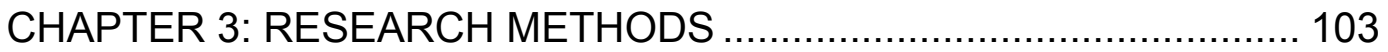

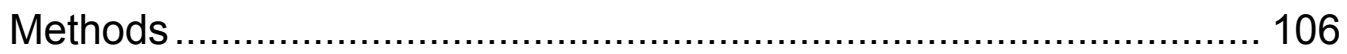

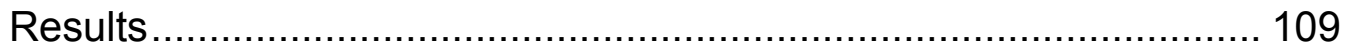

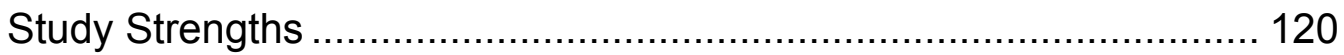

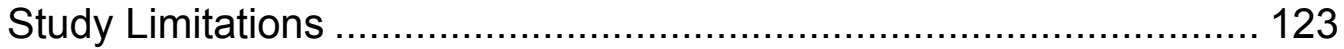

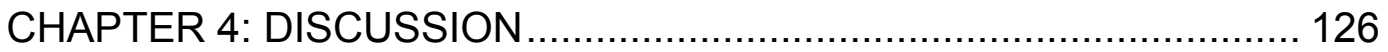

Implications for Policy ............................................................... 126

System Dynamics: Systems Thinking and Dynamic Modeling ....... 127

Mycobacterium Tuberculosis …............................................... 133

Nontuberculous Mycobacterium ................................................. 139

Policy Recommendations ........................................................... 143

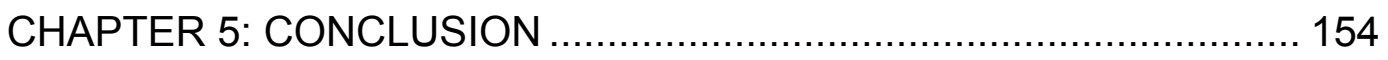

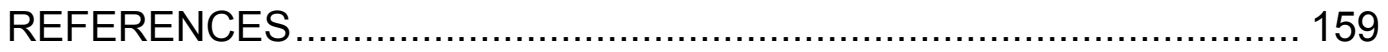

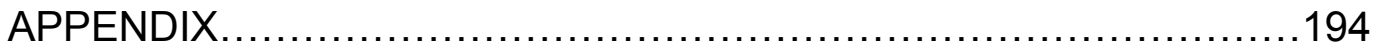

CURRICULUM VITA .......................................................... 


\section{LIST OF TABLES}

Table 1: Recommended Therapy for Drug Susceptible TB

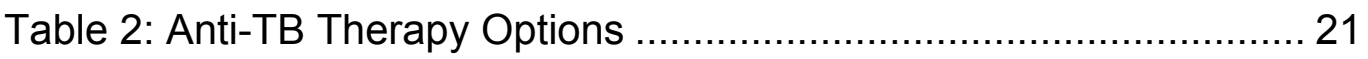

Table 3: Runyon Classification of NTM Species ................................... 27

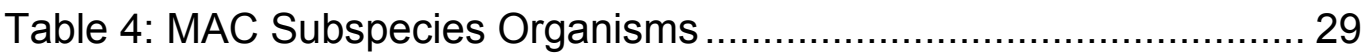

Table 5: Common NTM Human Pathogens ......................................... 29

Table 6: Pathogens Collected from Water and Clinical Samples ........... 38

Table 7: ATS/IDSA Official Definition of pulmonary NTM ...................... 43

Table 8: Program delivery costs for budgets and patients ..................... 66

Table 9: MDR-TB in Resource Scarce Care Settings ........................... 73

Table 10: NTM studies in the underdeveloped world............................ 94

Table 11: NTM and Cost in the Peer Reviewed Literature .................. 101

Table 12: Census Bureau Classification of the US by zone ................ 107

Table 14: Age distribution of pulmonary NTM and TB ...................... 111

Table 15: Gender Distribution of pulmonary NTM and TB.................. 112

Table 16: Geographical distribution of pulmonary NTM and TB .......... 113 
Table 17: Payer status of pulmonary NTM and TB

Table 18: Median income by zip code in patients with pulmonary NTM

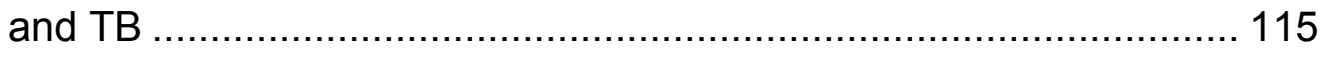

Table 19: Hospital charge due to mycobacterial disease …................ 116

Table 20: Cost figures for patients with pulmonary NTM and TB ........ 117

Table 21: Clinical outcomes of patients with pulmonary NTM and TB.. 120

Table 22: ICD-10 Billing Codes for NTM Infection ............................. 151 


\section{LIST OF FIGURES}

Figure 1: Global TB incidence 1990-2008 …...................................... 25

Figure 2: Global Average of NTM Species as a cause of disease ............ 30

Figure 3: NTM Species as a cause of disease in North America .............. 31

Figure 4: US population by age and sex in 2012 and projections ............. 51

Figure 6: Mycobacterial diseases cost trends .................................... 118

Figure 7: Epidemiological data for hospitalizations due to mycobacterial

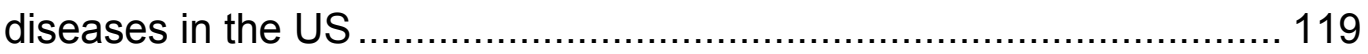

Figure 8: TB contact tracing model.................................................. 135 


\section{INTRODUCTION}

Mycobacterial diseases describe the infectious processes caused by one of many pathogens from the Mycobacteriaceae genus. The most notable of these pathogens is Mycobacterium tuberculosis (TB), which remains an important worldwide public health challenge. Another important pathogen is Nontuberculous mycobacteria (NTM) which has become an emerging public health problem. Both TB and NTM have many clinical presentations, but the most common manifestation of disease is pulmonary and both diseases manifest with similar symptomology. Both diseases are also associated with significant morbidity and mortality. Despite similarities, TB and NTM differ dramatically in terms of contemporary public health challenges. Recent advances in laboratory diagnostic techniques, the improvement of treatment regimens, and improvements in disease surveillance and control have allowed many developed countries to reduce the burden of TB. This is the case in the US in which TB related mortality has been in decline. Still, major challenges in TB care persist, and include the drug resistance and co-infection with HIV disease. Although TB in the developed world still impacts key populations, the global TB epidemic which have been shown to disproportionately affect developing countries in Asia and Africa. 
NTM, however, represents an emerging public health problem with increasing incidence and associated death rates that are projected to continue to increase in coming decades. The cost of care for both diseases is likely to reflect these challenges. The purpose of this study is to evaluate the cost burden of pulmonary TB and NTM in the US as well as to identify emerging trends using hospitalization data obtained from the Healthcare Cost and Utilization Project (HCUP), a database from the US Department of Health and Human Services. Data was collected between the years 2001 and 2012 in order to show changes over time for both diseases. The goal of this study was to demonstrate the clinical importance and cost of managing mycobacterial diseases in order to identify important considerations for future research as well as provide important data that could serve as decision support for the formation of health policy. 


\section{CHAPTER 1: OVERVIEW OF MYCOBACTERIAL}

\section{DISEASE}

Mycobacterium is defined as any one of the variety of both pathogenic and nonpathogenic organisms that are from the genus of the Mycobacteriaceae family. Currently consisting of nearly two hundred currently identified species, mycobacteria have several important and distinct characteristics. For starters, mycobacterium species are aerobic, which means these organisms rely on oxygen for survival. This also has important clinical implications for mycobacterial disease, which describes any disease process caused by pathogenic mycobacterium organisms. Pulmonary disease is the most common manifestation of mycobacterial diseases given the high availability of oxygen in the lungs, and symptoms appear the same in most cases regardless of the pathogen. However, mycobacterial diseases can occur at most any location. Mycobacterium organisms are slow growing, which impacts diagnostics given that cultures sometimes take weeks to form colonies in a laboratory setting. The slow growth of mycobacteria also has important clinical implications that can lead to a sometimes mild and chronic form of disease. Mycobacterium species are characterized by particularly thick cell walls that contribute to survival in different environments as well as resistance to many antibiotic 
therapies. Drugs used to treat mycobacterial diseases often involve alternative pathways of cell entry, as cell wall penetration is a common mechanism of action for many types of antibiotics. This results in fewer therapeutic options available to treat mycobacterial disease, and therapy is much longer in duration as compared to most other infections. Also, the cell wall is constructed of a waxy, lipid material that repels the dyes used in Gram staining resulting in a distinct appearance referred to acidfastness. This feature is unique to mycobacteria and helps support diagnostics given the slow culture growth of these organisms. Mycobacteria are referred to as bacillus, which is a term used to describe rod-shaped organisms. Their unique appearance also contributes to the recognition of mycobacteria in a laboratory setting. ${ }^{1,2}$ Infections caused by mycobacterium species tend to be chronic, difficult to diagnose, involve long complicated treatment courses, and represent a significant source of morbidity and mortality in the US and the world. ${ }^{3}$

\section{Mycobacterium tuberculosis}

Mycobacterium tuberculosis (TB) is the most common mycobacterium species that causes disease in humans. Discovered by German scientist Robert Koch in 1882 as part of the M. tuberculosis complex of organisms, TB includes diseases caused by $M$. africanum, $M$. bovis, M. microti, and M. canett, and M. tuberculosis. ${ }^{4}$ TB represents an important public health challenge on a global scale. 


\section{History}

TB has been believed to be a source of human disease for thousands of years, but the origin and pathogenic history of TB is not well understood. Several DNA studies of remains from humans and animals have attempted to piece together the epidemiological history of disease in world populations as well as the genetic evolution of $M$. tuberculosis organisms. For example, TB has been isolated in human remains collected from thousands of years ago from locations on both sides of the Atlantic Ocean. Evidence of the presence of TB in the western hemisphere consists of the discovery of the disease in a human mummy discovered in Peru from around 6,000 years ago. ${ }^{5}$ This finding was similar to those isolated from Egyptian mummies. ${ }^{6}$ Another discovery was made in the remains of an extinct bison estimated to be around 17,000 years old in North America. Identification of DNA fragments was conducted using the spoligotyping technique with direct DNA sequence determination, suggesting that cattle may have been one of the earliest vectors for disease transmission. ${ }^{7}$ In the eastern hemisphere, a study of samples obtained from ancient Syria identified the presence of TB in human remains; however, TB was not isolated from animal remains from the same site. This evidence suggests the presence of human disease prior to animals following domestication. ${ }^{8}$ Additionally, several studies have identified TB in the remains of humans from Europe, Asia and Africa. Many of these reports and subsequent DNA analyses can be found in the published work from Albert Zink and colleagues. In numerous studies, 
Zink has taken mummified and skeletal remains from different time periods and compared them with genetic material of TB obtained from present day samples. Comparing historical samples from southern Germany (1,400-1,800 AD), Hungary (600-1,700 AD), 9,10 and Egypt $(3,500-500 \mathrm{BC})$, Zink and researchers were able to further characterize mycobacterial DNA samples obtained from ancient Egypt. ${ }^{11}$

In the more recent history, TB is well described as a source of significant morbidity and mortality for centuries in Europe. Referred to by a number of names including consumption, the robber of youth, and the white plague, the disease was well described in both medicine and literature of the time. Doctors, writers and even poets described the characteristic features of the disease that included thin bodies secondary to wasting, pale complexion, and the association of the disease with the youth. ${ }^{12}$ In the seventeenth century, infection rates in London, England were reported at 1,000 per 100,000 people per year. ${ }^{13}$ During the eighteenth and nineteenth centuries, TB rates increased charactering the disease as an epidemic in Europe that disproportionately affected the poor. Although history reports incidents of the wealthy becoming infected with the disease, they were afforded the option to travel to more suitable climates, comforts and access to remedies. These options were not available to the poor, who were likely confined to substandard living conditions and subject to poor nutrition. These factors amplified the impact of the disease process in this population and most likely 
contributed to poor outcomes and an increase in death rates. ${ }^{14}$ By the nineteenth century, TB was attributable to $25 \%$ of all mortality in Europe. Interestingly, infection rates declined at the turn of the twentieth century prior to the discovery of anti-TB drugs. This decrease in TB infection rates is believed to be a result of improvements in standards of living in cities, which includes housing, nutrition and rising income. ${ }^{15}$ As a result of the advent of anti-TB drugs coupled with public health policies focused on treatment and prevention, TB in the twentieth century became irrelevant. In fact, some experts of the time believed that TB could be eradicated like other infectious diseases such as smallpox. ${ }^{16}$

At the close of the twentieth century, TB reemerged as a major global epidemic. Among the factors contributing to the sharp resurgence in TB rates was the worldwide HIVIAIDS pandemic coupled with the onset of drug resistance. TB having become a recurring global health crisis stimulated renewed interest in research and development in many areas of the disease, resulting in recent advancements in diagnostics, treatments, and improving the understanding of clinical and nonclinical factors impacting human disease. ${ }^{17}$ However, because mortality rates sharply declined for a time, fewer resources were diverted to new drug development, diagnostics and vaccine studies for several decades. As a result, the medical community continues to rely upon outdated therapies and diagnostic technology in care settings in both developed and low resource countries, leaving much of the world ill equipped to address 
emerging needs. ${ }^{18,19}$

\section{Clinical Features}

The natural history of TB infection begins with exposure followed by the onset of latent disease. Latent TB describes the colonization of the organism in body. Latent TB rarely presents with symptoms as the mycobacterial pathogens remain dormant until an opportunity presents itself to overtake a weakened immune system and multiply. It is common for active infection to occur years later following a major change to the host's health status, particularly the immune system. However, the activation of disease sometimes occurs for no known reason, resulting in progression from latent to active disease being sometimes unpredictable.

Screening for latent disease can be part of an effective prevention strategy by treating latent infection thus preventing the activation of disease.

Treating latent disease can also be an important strategy in reducing further transmission. Testing for dormant infection can be done using the tuberculin skin test (TST) or blood assay tests including the QuantiFERON and T-SPOT. ${ }^{20,21}$

The next phase in TB is the activation of disease. Pulmonary TB is the most common manifestation of disease with classic symptoms including chronic productive cough, hemoptysis, fevers and/or chills, night sweats, and weight loss. However, TB infection can occur at virtually any position in the body. Atypical presentations, which is commonly referred to as extrapulmonary TB, describes any presentation of TB infection 
outside of the lungs. ${ }^{22}$ In these cases, symptomology can be diverse with many symptoms having been described in the medical literature for each site of infection. Most common extrapulmonary TB sites of infection include lymphadenitis, pleural space, septic arthritis, osteomyelitis, central nervous system, abdomen, and disseminated disease. Extrapulmonary TB has become more common since the beginning of the HIVIAIDS pandemic, with immunosuppression associated with advanced AIDS being a primary risk factor. In fact, more than half of AIDS patients develop extrapulmonary TB. Further, the management of extrapulmonary TB in HIV infected hosts can present treatment challenges given the higher likelihood of drug-drug interactions, but treatment response in HIV infected hosts seems overall favorable without any measurable increased risk of infection relapse. ${ }^{23}$

Host factors play a critical role in susceptibility to infection as well as disease presentation, some of which are not well understood. Young adult males, for example, have been shown to have a higher likelihood of developing TB than other age groups and more so than females. Healthcare workers are another important risk group given their much higher likelihood of exposure, especially those working with populations with higher TB prevalence or in specialty clinics in which TB patients are being treated. Other host factors can be explained by immune suppression. ${ }^{24}$ Activation of TB can occur secondary to several health conditions and diseases that suppress the immune system, the use of 
certain drug therapies that suppress the immune system as part of cancer treatment, post-transplant therapy or immune modulation therapy such as antitumor necrosis factor- $\alpha$ (TNF- $\alpha$ ) drugs commonly used to treat autoimmune diseases. Also, the presence of several underlying diseases are a common facilitator of active TB infection in many parts of the world such as pulmonary diseases, diabetes mellitus, ${ }^{25}$ and other conditions that have been shown to affect the immune system. Behavioral factors can also play an important role such as diet and malnutrition, alcoholism, and smoking tobacco, the latter of which the World Health Organization (WHO) attributes to $20 \%$ of TB cases. ${ }^{26,27}$ Emerging science has also pinpointed genetic susceptibility factors, some of which are being explored for the purpose of developing personalized therapies and vaccines. ${ }^{28,29}$

\section{Transmission}

The transmission patterns of TB have remained a point of inquiry in historical and current studies. The transmission of disease person to person is well described. Although the highly contagious nature of TB pathogens allows the disease to be transmitted with only small amounts of airborne respiratory fluids propelled through the air via coughing and sneezing, the reasons TB is transmitted to some and not others following exposure is not established. ${ }^{32}$ Also, transmission pathways between humans and animals, and vice versa, are not well understood. Historical studies have sought to understand this pathway in ancient populations in which evidence of disease was identified in both animal and in human 
populations, but the transmission from one to another following the domestication of animals in prehistoric populations has not been fully documented. More recent history demonstrates a strong relationship between human and animal populations. A study published by Davies traces TB reports in nineteenth century slaughterhouses, cow's milk during Victorian times, and possible transmission from cows to humans during urbanization through infected milk. In fact, this transmission pathway has been described as an important facilitator of the resurgence of disease in the eighteenth and nineteenth centuries held by several researchers, proposing that TB infected milk in Great Britain was responsible for the epidemic beginning in the Victorian Era through World War II. ${ }^{33,34}$ Further, Davies estimates TB in the food supply remains an important risk in developing countries, particularly when food production occurs within the bounds of densely populated cities. ${ }^{35}$ Among the $M$. tuberculosis complex of organisms, $M$. bovis has been the organism most often associated with disease in cattle. Although few cases of M. bovis have been identified through diagnostic methods, some researchers suspect this number is underreported given limitations in diagnostic techniques in correctly sampling specimens. Regardless of the potential clinical importance of $M$. bovis, $M$. tuberculosis remains the most important cause of TB infection worldwide. ${ }^{36}$

Additionally, some have questioned the animal to human and human to animal transmission pattern of other M. tuberculosis complex 
organisms as well as the role of other animal species. Several case reports are present in the literature of TB having been found in companion animals, but the transmission pathway remains unclear. A case report of a nine-year-old pet dog was found to have had tuberculomas in the heart, which is a rare manifestation of TB in humans and presumably animals. Case reports generally describe the presentation of disease as pulmonary or lymphadenopathy. This report notes that TB infection was not present in any members of the household. ${ }^{37}$ Another case study identified TB infection in a companion dog using Polymerase chain reaction (PCR) and spoligotyping at necropsy. This study noted that TB infection was not found in household members, but the diseases may have been transmitted from the dog to veterinary personnel during necropsy. ${ }^{38} \mathrm{~A}$ recent case study from Brazil described TB infection and mortality in a pet dog following the occurrence of TB in the household. ${ }^{39}$ Another Brazilian case study noted disseminated disease in a 12-year-old pet dog diagnosed following the death of one the members of her household secondary to pulmonary TB. The dog in question was noted to have been in particularly close contact with the diseased, having been observed licking respiratory secretions during his illness. ${ }^{40}$

More collaborative and multi-disciplinary research is needed in order to better understand the scope of animal to human transmission and prevent the transmission of disease through medical and public health intervention. The future of research in this area will depend upon 
improvements in diagnostic techniques, application of more advanced discriminatory genotyping tools, and collaboration with veterinary researchers. ${ }^{41}$

Further, environmental transmission of TB has not been ruled out as a possibility given the presence of pathogenic samples identified in soil and water samples. In a study conducted in metropolitan Tehran, TB was identified in 11 of 800 soil samples and 71 of 700 water samples collected. Following six months after sampling, $36 \%$ of samples from soil and $8.4 \%$ of samples from water remained culturable. This finding implies that TB organisms remain viable in environmental sources for extended periods of time, increasing the possibility of transmitting the disease to human and animal populations. ${ }^{42}$

\section{Diagnosis}

The process of diagnosing active TB begins with the evaluation of symptomology and host factors before proceeding to radiological and microbiological findings. Pulmonary TB has classic symptoms and characteristic cavitary lesions usually appearing in the upper lobes of the lungs visible on radiological exam. ${ }^{43}$ However, abnormal host factors can undermine classic disease appearance in the lungs. Host factors also are an important indicator of the presentation of extrapulmonary disease. ${ }^{44}$

Laboratory findings represent another important factor in disease diagnosis. The diagnosis of TB usually involves a two-step process: 
smears that determine the presence mycobacterium species and follow up cultures or molecular testing that are used for species identification. Ideally, a third step in the process should include drug susceptibility testing (DST).

The process begins with smears, which involves staining clinical samples in order visualize appearance of the organism. Characteristic features of mycobacterium species are acid-fastness due to the unusual thick and lipid cell wall of the organism. Also, mycobacterium species are rod shaped, which would be visualized following smear staining. A number of smear methods are currently used. Fluorescence microscopy represents one of the first and most common laboratory techniques. This method involves the use of flourochrome dyes on the samples along with halogen or high-pressure mercury vapor lamps in order to visualize the results. However, the use of fluorescence microscopy relies on reliable access to electricity and related infrastructure, which is not realistic in some settings. Conventional smear microscopy using the Ziehl-Neelsen technique has been the most common method used in low to middle resource countries for this reason. Although this method has been well proven and represents a cost effective smear test in many care settings, smear microscopy requires high quality samples. ${ }^{45}$ Also, the sensitivity and specificity decrease when diagnosing extra-pulmonary disease and this method is less proven in pediatric populations and in patients with compromised immune systems. ${ }^{46,47}$ 
Because smears only have the capacity to identify mycobacterium, follow up tests are necessary for species identification. Mycobacteria cultures can be performed using liquid or solid media. Liquid cultures involve an automated process with a relatively quick turn around time with high sensitivity rates. However, the risk of contamination is higher for liquid culture due to the presence of mycobacterium species in water. ${ }^{48}$ Polymerase chain reaction (PCR) is an alternative testing method using a DNA amplification technique. ${ }^{49}$ The major advantage of PCR is results are reported usually within a couple of days. High-performance liquid chromatography (HPLC) is another molecular method that has been used for identifying mycobacterium species. Having been first introduced in the 1960s, HPLC has been standard procedure at the CDC Mycobacteriology Reference Laboratory since $1990 .{ }^{50,51}$ Among the major advantages of HPLC is that species identification can be as immediate as a few hours. However, HPLC relies on high quality specimens and requires highly trained laboratory personnel to perform the procedure. Further, this technique is expensive as compared to cultures. This has important implications for use in many low resource, high burden TB countries. In many of these clinical settings, laboratory methodology relies only upon smears, and follow up studies involving species identification and drug susceptibility testing is not done. ${ }^{52}$ Also, cultures and molecular testing may be done in cases of negative results following smear staining when mycobacterial diseases are highly suspected. All laboratory techniques 
rely on quality specimens, and have differing capabilities depending on the specimen source. Given that pulmonary infection is the most common manifestation of mycobacterial disease, laboratory testing techniques generally have a better yield using respiratory samples. Also, invasive collection techniques for respiratory samples such as bronchoscopy are considered higher quality specimens. ${ }^{53,54}$

The final step that follows smears and species typing is drug susceptibility testing (DST). DST describes the process of testing laboratory isolates against common antimicrobial agents in order to optimize therapy. This step is especially important in cases in which resistant disease is suspected following the reconciliation of patient characteristics that imply a risk for resistant disease. These factors may include a patient history of treatment failure or having been born or visited regions of the world in which drug resistance is common. ${ }^{55}$ Another consideration for DST involves the risk of infection from other mycobacterium species.

However, diagnostic challenges persist for many reasons. First, the slow growth of mycobacterium organisms often requires therapy initiation in suspected cases prior to official diagnosis. In some cases, patients may be treated improperly for another disease process such as community-acquired pneumonia, which represents inappropriate antibiotic and healthcare resource use. Also, the slow development of new diagnostic technology remains a persistent challenge. Given the low 
prevalence of TB until the end of the twentieth century when global resurgence occurred, research and development in TB laboratory methodology all but halted along with drug therapies and vaccine studies. Only recently has research and development in the area of TB produced results that have improved laboratory methods and made available new treatment options. The challenges this creates involve not only diagnostics, but also therapy choice. Slow and difficult microbiological testing often results in the initiation of empiric therapies based on the clinical suspicion of clinicians in terms of organism and drug susceptibility as confirmation of infection may occur weeks later. ${ }^{56,57,58}$

Despite the numerous options for laboratory studies, access to many of these techniques is unavailable in many care settings. A recent report by UNITAID, an organization developed to address common global health concerns in a cost effective approach, is hopeful regarding the future of diagnostic technology. First, the recent introduction of Xpert $\circledast$ Mycobacterium TB/Rifampin (MTB/RIF) has been an important development that has been encouraging for the future availability of new technology. Xpert MTB/RIF is a new diagnostic technique that replaces multi-step process of smears, cultures and susceptibility testing to first line drug rifampin is encompassed in one test cartridge. In 2014, more than 10 million Xpert MTB/RIF test cartridges were purchased by the public sector in 116 of the 145 countries eligible for concessional pricing. UNITAID reports that, in addition to Xpert MTB/RIF, a pipeline of 
molecular diagnostics or nucleic acid amplification tests are in development from over 50 companies. However, the report noted that new diagnostic methods will struggle to reach scale. Also, other challenges including long treatment courses persist with no obvious new regimens with shorter duration of therapy in the pipeline. UNITAID also emphasized the importance of developing diagnostics that do not rely on the availability of sputum samples for select situations, particularly for the diagnosis of disease in pediatric populations. Given that TB disproportionately affects low resource countries in greater prevalence, UNITAID also raised concerns regarding the availability of new technologies to low resource countries specifically. ${ }^{59}$ Other reports examining the future landscape of TB diagnostics have raised similar concerns regarding the availability and affordability of TB diagnostic technology to the countries in greatest need. ${ }^{60}$

\section{Treatment}

Treatment for TB presents a number of important challenges.

First, anatomical properties of mycobacterium species including an abnormal thickness of the cell wall as compared to other bacterial organism makes TB resistant to most antibiotics. This leaves few treatment options relative to the several classes of currently available antibiotics. Also, standard treatment for active infection involves a regimen ranging from six months to nine months in duration, or four months following culture conversion. During treatment courses, patients 
must make regular visits to providers to ensure medication compliance as well as measure clinical response. Providers must also prevent treatment interruptions or abandonment by ensuring patients maintain the ability to be compliant, or transfer treatment in the event of relocation. The failure of patients to complete treatment has important implications for the continued transmission of disease as well as the facilitation of drug resistance. Another important factor is TB treatment is the management of drug therapies relative to adverse reactions and comorbid conditions. Long treatment courses compound the risk of side effects and adverse events. As a result, standard of care involves regular blood monitoring in order to identify toxicity and other potentially serious side effects. Also, TB drugs have the potential to interact with treatment for several comorbid conditions, which also represents an important aspect of medical management. For these reasons, providers must be vigilant in maintaining patient contact during treatment courses. This can be particularly challenging when appropriate treatment requires compliance from patient populations known for incompliance.

Standard treatment for TB may include any of the following firstline drugs: isoniazid, rifampin, rifabutin, rifapentine, pyrazinamide, or ethambutol. Table 1 outlines the recommended therapy for adults ages 15 years and older with active infection based on expert guidelines published in a joint report by the American Thoracic Society/Centers for Disease Control and Prevention/Infectious Diseases Society of 
America. ${ }^{61}$

Table 1: Recommended Therapy for Drug Susceptible TB

\begin{tabular}{|c|c|c|c|c|c|}
\hline $\begin{array}{l}\text { First-Line } \\
\text { Therapies }\end{array}$ & Preparation & $\begin{array}{l}\text { Daily } \\
\text { Dose }\end{array}$ & $\begin{array}{l}\text { Weekly } \\
\text { Dose }\end{array}$ & $\begin{array}{c}\text { 2x/week } \\
\text { Dose }\end{array}$ & $\begin{array}{c}\text { 3x/week } \\
\text { Dose }\end{array}$ \\
\hline Isoniazid & $\begin{array}{l}\text { Tablets (50 } \\
\text { mg, } 100 \mathrm{mg} \text {, } \\
300 \mathrm{mg} \text {; elixir } \\
\text { ( } 50 \mathrm{mg} / 5 \mathrm{~mL} \text { ); } \\
\text { aqueous } \\
\text { solution (100 } \\
\mathrm{mg} / \mathrm{mL} \text { ) in IV } \\
\text { or intra- } \\
\text { muscular } \\
\text { injection }\end{array}$ & $\begin{array}{l}5 \mathrm{mg} / \mathrm{kg} \\
(300 \mathrm{mg})\end{array}$ & $\begin{array}{l}15 \mathrm{mg} / \mathrm{kg} \\
(900 \mathrm{mg})\end{array}$ & $\begin{array}{l}15 \mathrm{mg} / \mathrm{kg} \\
(900 \mathrm{mg})\end{array}$ & $\begin{array}{l}15 \mathrm{mg} / \mathrm{kg} \\
(900 \mathrm{mg})\end{array}$ \\
\hline Rifampin & $\begin{array}{l}\text { Capsule } \\
\text { ( } 150 \mathrm{mg}, \\
300 \mathrm{mg}) ; \\
\text { powder may } \\
\text { be } \\
\text { suspended } \\
\text { for oral; } \\
\text { aqueous } \\
\text { solution for } \\
\text { IV injection }\end{array}$ & $\begin{array}{l}10 \mathrm{mg} / \mathrm{kg} \\
(600 \mathrm{mg})\end{array}$ & & $\begin{array}{l}10 \mathrm{mg} / \mathrm{kg} \\
(600 \mathrm{mg})\end{array}$ & $\begin{array}{l}10 \mathrm{mg} / \mathrm{kg} \\
(600 \mathrm{mg})\end{array}$ \\
\hline Rifabutin & $\begin{array}{l}\text { Capsule } \\
(150 \mathrm{mg})\end{array}$ & $\begin{array}{l}5 \mathrm{mg} / \mathrm{kg} \\
(300 \mathrm{mg})\end{array}$ & & $\begin{array}{l}5 \mathrm{mg} / \mathrm{kg} \\
(300 \mathrm{mg})\end{array}$ & $\begin{array}{l}5 \mathrm{mg} / \mathrm{kg} \\
(300 \mathrm{mg})\end{array}$ \\
\hline Rifapentine & $\begin{array}{l}\text { Tablet (150 } \\
\text { mg, film } \\
\text { coated) }\end{array}$ & & & $\begin{array}{l}10 \mathrm{mg} / \mathrm{kg} \\
\text { (continuati } \\
\text { on phase) } \\
(600 \mathrm{mg})\end{array}$ & \\
\hline Pyrazinamide & $\begin{array}{l}\text { Tablet (500 } \\
\text { mg, scored) }\end{array}$ & $\begin{array}{l}\text { Weight- } \\
\text { based } \\
\text { dosing }\end{array}$ & & & \\
\hline Ethambutol & $\begin{array}{l}\text { Tablet }(100 \\
\mathrm{mg}, 400 \mathrm{mg}\end{array}$ & $\begin{array}{l}\text { Weight- } \\
\text { based } \\
\text { dosing }\end{array}$ & & & \\
\hline
\end{tabular}


For patients with resistance or toxicity to first line agents, TB treatment courses may consist of a host of other less effective or less proven drug options. These regimens may be used following a treatment failure, resistance reported following DST performed at diagnosis, or following a clinical review of medical history that is suspicious for drug resistance. Factors such as recent travel from regions with high resistance patterns or medical history with previous regimens and/or treatment failures may impact choice in therapy. Table 2 shows a complete grouping of anti-TB therapy. ${ }^{62}$

Table 2: Anti-TB Therapy Options

\begin{tabular}{|ll}
\hline Group 1: First line (Use all possible drugs) \\
\hline Isoniazid & Ethambutol \\
Rifampin & Pyrazinamide
\end{tabular}

Group 2: Flouroquinolones (Use only one, agents share genetic targets) Levofloxacin Moxifloxacin

Ofloxacin

Group 3: Injectable (Use only one, agents share similar genetic targets) Capreomycin Amikacin Kanamycin Streptomycin

Group 4: Less effective second line drugs (Use all possible drugs if necessary)

Aminosalcylic acid (acid salt)

Group 5: Less effective or less proven drugs (Use all necessary drugs if there are less than four from the other groups)

Bedaquiline

Linezolid

Delamanid Imipenem/cilastatin

Clofazimine Meropenem

Amoxicillin with clavulanate Clarithomycin 
Drug resistance is a common problem affecting the treatment of many infectious diseases and is most commonly a consequence of overuse and inappropriate use of common drug therapies. In TB care, drug resistance is among the most important challenges, which is a worldwide problem affecting both developed and underdeveloped countries. Multi-drug resistant TB (MDR-TB) is characterized by resistance to first line drugs isoniazid and rifampin. Extreme-drug resistance TB (XDR-TB) is characterized by resistance to first line drugs isoniazid and rifampin, in addition to any fluoroquinolone and one of the following three injectable second-line drugs: amikacin, kanamycin, and capreomycin. Scant reports of totally drug resistant TB (TDR-TB) have been made in which strains have demonstrated resistance to all drugs used for TB treatment. ${ }^{63,64,65}$

Drug resistance creates greater challenges in the developing world for several reasons. First, standard laboratory techniques are often limited in resource scarce countries, so susceptibility testing may not be standard or available. In these cases, resistance may be diagnosed following a treatment failure, which represents more cost to the healthcare system as well as a longer treatment course and a greater risk of adverse events. ${ }^{66}$ Also, second line therapies may be limited and more costly, which is particularly challenging when managing care in underdeveloped countries with limited resources. According to the WHO, 480,000 cases of MDR-TB were reported in 2014 worldwide. An estimated 10\% of MDR cases were 
XDR-TB. More than half of all resistant cases were in China, India and Russia. ${ }^{67}$

\section{Epidemiology}

The WHO characterizes TB as a worldwide epidemic with cases having been documented in almost every country around the world. In 2014, nearly 10 million people developed active disease and 1.5 million died as a result. An estimated one third of the world's population is currently infected, most of which with latent disease. ${ }^{68}$ Public health policy in the US and abroad has focused on treatment and disease surveillance of pulmonary TB. These efforts coupled with improvements in diagnostics, treatment, and prevention have reduced the public health impact of TB in the US and other western nations. In the underdeveloped world, TB remains an important public health challenge.

The relationship between TB and poverty is well described. Contributing factors driving the transmission of TB is these settings include high population density along with malnutrition, concomitant medical conditions that affect immunity, and behavioral factors such as smoking and alcohol use. For these reasons, the burden of new cases falls disproportionately on poor countries located in Southeast Asia, Western Pacific and Africa. In fact, $80 \%$ of new cases occurred in the same 22 countries in 2014, including South Africa, Nigeria, India, Pakistan, Indonesia and China to name a few. Also, over 95\% of TB related deaths occur in low to middle resource countries. ${ }^{69}$ In many of 
these countries, HIVIAIDS represents a critical factor predisposing large segments of the population to TB infection. According to some reports, HIVIAIDS is believed to be responsible for a three to five times increase in the incidence of TB infection in high HIV prevalence countries located in sub-Saharan Africa. ${ }^{70,71}$ TB infection in HIV/AIDS infected hosts often presents with more aggressive disease as well as extrapulmonary involvement. In fact, the atypical presentation of TB occurs in more than half of patients with advanced HIVIAIDS. Co-infected hosts also are more likely to experience complications as well as medication interactions, and TB related mortality in this population is a common outcome. ${ }^{72}$ A 2014 WHO report claims that one in three deaths of HIV positive patients can be attributed to TB, emphasizing the particularly devastating role HIV plays in TB in countries with high HIVIAIDS prevalence. The WHO also reports that HIV infected patients are 20 to 30 times more likely to progress to active disease from latency as compared to the general population. ${ }^{73}$ The impact of HIVIAIDS is easily illustrated in the figure below outlining a comparison of worldwide TB incidence showing data from high-income countries verses other world regions including a stratification of data from Africa separately reported based on high HIVIAIDS prevalence. ${ }^{74}$ 


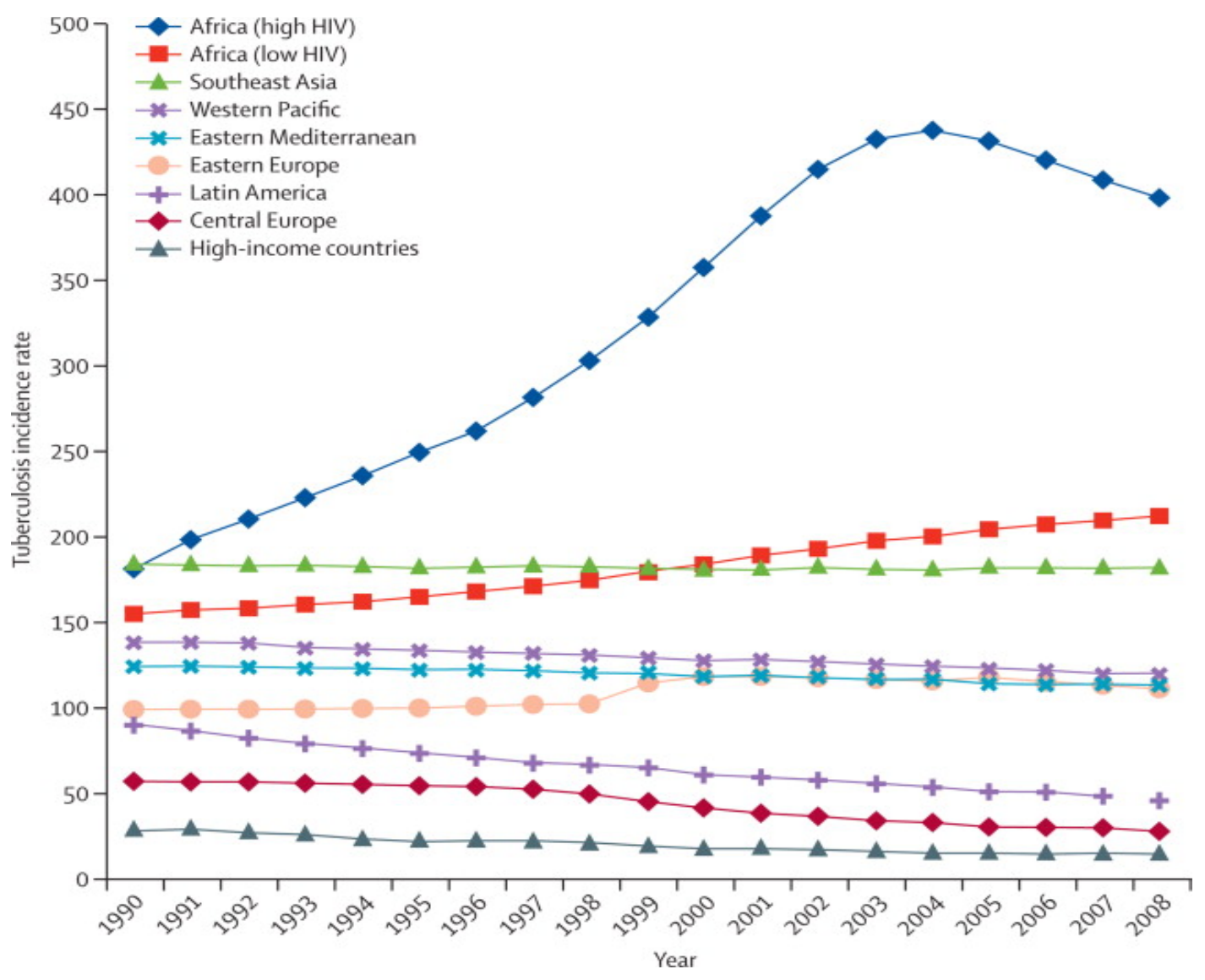

Figure 1: Global TB incidence 1990-2008

The availability of current diagnostic methods and anti-TB therapies has been one of the most important developments in addressing TB as a worldwide global health concern. An estimated 43 million lives were saved between the years 2000 and 2014 as a result of TB diagnostics and treatment. ${ }^{75}$

\section{Nontuberculous mycobacteria}

Nontuberculous Mycobacterium (NTM) describes a group of organisms ubiquitous to the environment that have been associated with disease in both humans and animals. Recent reports indicate that NTM is 
an emerging pathogen in many parts of the world, but particularly in developed countries in North America, Europe, Asia and Australia. ${ }^{76}$ In the US, NTM rates are sharply rising in prevalence, particularly in the older population of adults. ${ }^{77,78}$ NTM is a source of significant morbidity in the US. Patients with NTM infection require extensive healthcare resources including hospitalizations, frequent outpatient visits, and complicated therapy with associated treatment challenges. NTM is also an increasingly important cause of mortality in the US. ${ }^{79,80,81}$

\section{History}

NTM was first identified in the 1950 s by Ernest Runyon. A botanist who published his findings in 1959, Runyon categorized the newly discovered species based on growth rates, colony morphology, and pigmentation. ${ }^{82}$ Prior to Runyon's discovery, the history of NTM as a human pathogen remains unknown.

The Runyon system categorizes NTM species into 2 primary categories: slow growing and fast growing. Slow growing NTM are further organized into three subcategories based on the pigment production. ${ }^{83}$ The contemporary Runyon classification system consists of a considerably higher number of NTM species as more continue to be discovered. Currently, more than 150 species of NTM have been identified. ${ }^{84,85}$ As advances in laboratory methods have evolved in recent years, the Runyon classification system based on growth rates and pigment production has become less relevant. New rapid culturing techniques including DNA 
probes and high-pressure liquid chromatography do not abide by the Runyon classification system. Also, the Runyon classification does not reflect unique clinical features of each pathogenic NTM species. ${ }^{86,87}$

Table 3: Runyon Classification of NTM Species

\begin{tabular}{|c|c|c|c|c|}
\hline \multicolumn{4}{|c|}{$\begin{array}{l}\text { Slow Growers (Colonies may take } \\
\text { up to } 8 \text { weeks to form) }\end{array}$} & \multirow{2}{*}{$\begin{array}{l}\text { Fast } \\
\text { Growers } \\
\text { (Colonies } \\
\text { grow within } \\
5 \text { days) } \\
\\
4 \\
\end{array}$} \\
\hline Runyon & 1 & 2 & 3 & \\
\hline Classification & Photochromogens & Scotchromogens & Nonchromogens & Rapid Growers \\
\hline Pigment & $\begin{array}{l}\text { Produces } \\
\text { yellow-orange } \\
\text { pigment } \\
\text { following } \\
\text { exposure to light }\end{array}$ & $\begin{array}{l}\text { Produces } \\
\text { yellow-orange } \\
\text { pigment in light } \\
\text { and dark }\end{array}$ & $\begin{array}{l}\text { Produces no } \\
\text { pigment }\end{array}$ & $\begin{array}{l}\text { Produces no } \\
\text { pigment }\end{array}$ \\
\hline Species & $\begin{array}{l}\text { M. kansasii, } M \text {. } \\
\text { marinum, } M \text {. } \\
\text { asiaticum, and } \\
\text { M. simiae }\end{array}$ & $\begin{array}{l}\text { M. gordonae } \\
\text { and } M \text {. } \\
\text { scrofulaceum }\end{array}$ & $\begin{array}{l}\text { M. avium, } M . \\
\text { intracellulare, } \\
\text { and } M . \\
\text { ulcerans }\end{array}$ & $\begin{array}{l}\text { M. fortuitum, } M \text {. } \\
\text { peregrinum, } M \text {. } \\
\text { abscessus, } M \text {. } \\
\text { chelonae, and } \\
M \text {. } \\
\text { thermoresisibile }\end{array}$ \\
\hline
\end{tabular}

Despite recent advances in NTM research, this field of mycobacteria remains understudied. A lack of support in the form of international grants as suggested by one study is responsible for the deficit in clinical and basic science research in the area of NTM ${ }^{65}$ In the US, only 5 of the 228 grants available from the National Institute of Health (NIH) include sponsorship for research in NTM. ${ }^{88}$ Therefore, the field of NTM research represents an important unmet need relative to the rising epidemiology and cost in the US. 


\section{Clinical Features}

The majority of NTM cases manifest in the form of pulmonary disease, as is the case with TB infection. Extrapulmonary NTM, or NTM cases that present in the form of infection outside of lung disease, is estimated to occur in the US in up to $10 \%$ of cases. ${ }^{89}$ NTM infections have the potential to involve any site in the human body. Atypical presentations cause a number of infections including lymphadenitis, septic arthritis, osteomyelitis, skin and soft tissue infections, and disseminated disease..$^{90,91,92}$

NTM disease can be caused by a number of different species as many are considered pathogenic to humans. Currently, more than 150 species of NTM have been described, with new ones being routinely discovered. ${ }^{9}$ The most common NTM pathogens associated with human infection are the species associated with Mycobacterium avium complex (MAC). MAC describes infections caused by two primary organisms, $M$. avium and $M$. intracellulare, as well as any one their genetically similar subspecies. In the US, MAC accounts for up to $80 \%$ of pulmonary NTM infections according to some reports..$^{93,94,95}$ Other reports classify more than half of NTM cases in the US as MAC, and global figures are only slightly less. Molecular sequence data have identified 10 subspecies of MAC as listed in the table below. ${ }^{96}$ 
Table 4: MAC Subspecies Organisms

\section{Mycobacterium Avium Complex Subspecies Organisms}
M. avium
M. intracellulare
M. homisissuis
M. bouchedurhonense
M. silvaticum
M. timonense
M. paratuberculosis
M. arosiense
M. colombiense
M. marseillense

Although MAC represents one of the most important causes of NTM disease, many NTM species have been associated with human diseases. See Table 5 for a listing of common NTM pathogens that have been strongly associated with infection in humans. ${ }^{97}$ Table 5: Common NTM Human Pathogens

\begin{tabular}{|ll|}
\hline \multicolumn{2}{|c|}{ Common NTM Human Pathogens } \\
\hline M. avium complex (MAC) & M. porcinum \\
M. kansasii & M. conceptionense \\
M. porcinum & M. phlei \\
M. lentiflavum & M. austroafricanum \\
M. monacense & M. elephantis \\
M. abscessus & M. parascrofulaceum \\
M. thermoresistibile & \\
\hline
\end{tabular}

According to a recent study outlining the epidemiology of NTM species as a cause of pulmonary disease, the geographical variation of 
NTM varies considerably between sample data from six continents.

Overall, MAC still represents the most common cause of pulmonary infection. See the figures below for a representation of the most common NTM species associated with disease in humans based on global averages as well as figures for North America. These data demonstrate both the clinical importance of MAC on both a global scale and in North America, as well as the other NTM pathogens as important causes of disease. Also, these data emphasize the importance of rapidly growing mycobacteria (RGM) as a cause of disease in North America. ${ }^{98}$ The most common RGM species are M. fortuitum, $M$. abscessus, and $M$ chelonae. ${ }^{99}$

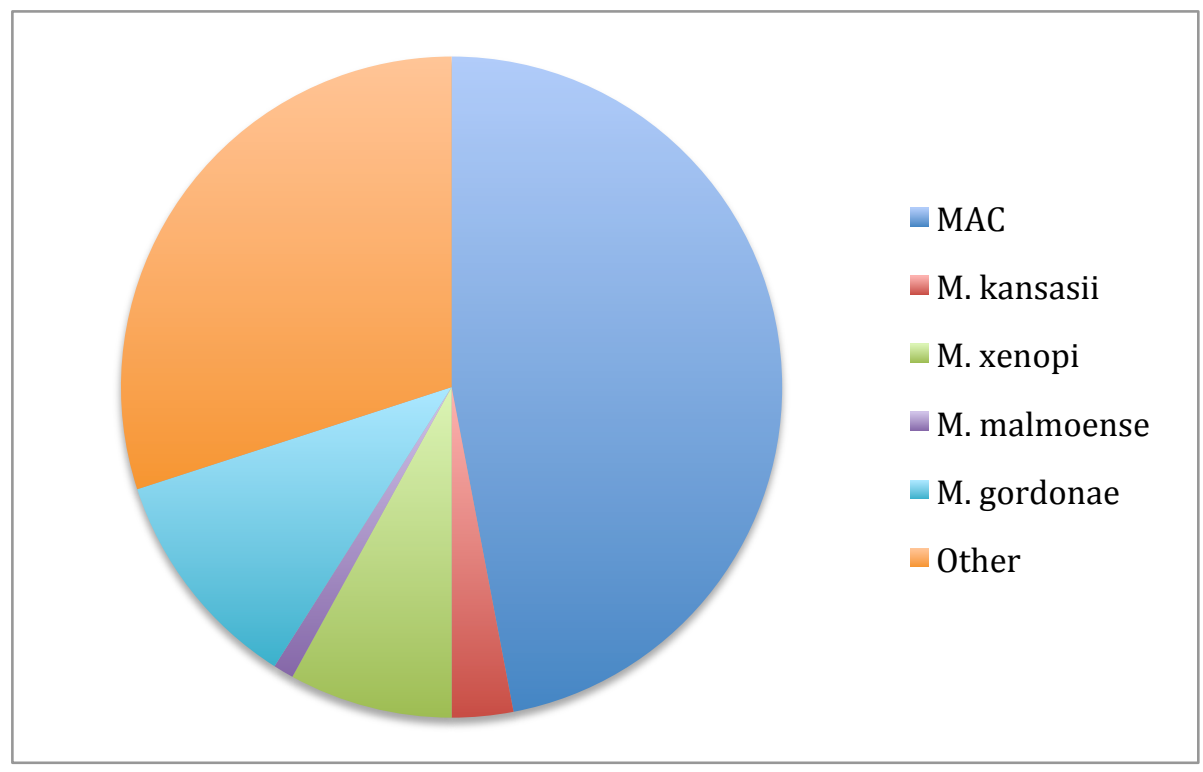

Figure 2: Global Average of NTM Species as a cause of disease 


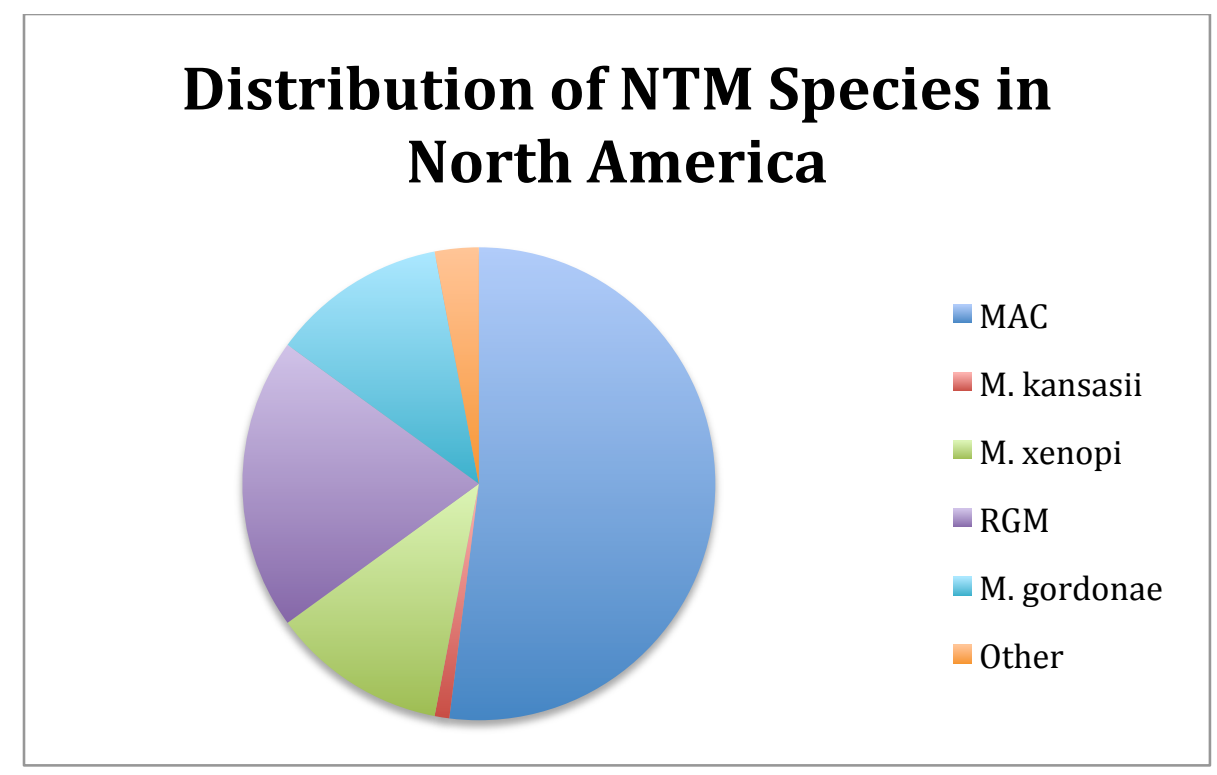

Figure 3: NTM Species as a cause of disease in North America

The amount of NTM to which one has been exposed has also been shown to predict the likelihood of disease. However, the infectious dose for NTM infection remains unknown but current estimates demonstrate this at anywhere between $10-10^{2}$ units. ${ }^{100}$ In a mouse model, $M$. ulcerans infections identifiable by swelling occurred following exposure to a dose of $10^{3}-10^{4}$ colony-forming units. ${ }^{101}$ This relationship has not been tested in other NTM species and hosts, including humans.

As is the case with TB infection, host factors play a critical role in susceptibility and clinical features of NTM infections. NTM is generally considered opportunistic pathogens, which means that some level of immunodeficiency or preexistent pulmonary disease usually exists in patients with NTM. ${ }^{102}$ Four categories of well-defined susceptibility for NTM infection have been identified. ${ }^{103}$ First, structural or preexisting 
pulmonary diseases such as cystic fibrosis, chronic obstructive pulmonary disease (COPD), and bronchiectasis have been strongly associated with the risk of developing many types of infectious lung diseases including NTM. ${ }^{104}$ Second, patients with autoimmune disorders who are being treated with antitumor necrosis factor- $\alpha$ (TNF- $\alpha)$ drugs are at risk for developing NTM as well as many other opportunistic infections. Third, HIV infected persons with AIDS are also at an increased risk for developing NTM along with many other opportunistic infections. In fact, a CD4+ T cell count of less than 50 cells/ $\mu \mathrm{L}$ is associated with increased risk of disseminated NTM disease. Fourth, patients with genetic syndromes involving mutations in the interleukin-12 or interferon $y$ pathways are also at risk for developing opportunistic infections including NTM. Mutations in these pathways are associated with both autoimmune disorders as well as immune suppression. ${ }^{105,106,107,108}$

In addition to these defined risk groups, solid organ transplant recipients also have increased risk of NTM disease for several reasons. Post-transplantation immunosuppressive therapy increases the likelihood of clinical disease from environmental exposures. ${ }^{109}$ Also, underlying lung disease in lung transplant patients place patients at a higher risk for NTM infection during the pre-transplant period. Possible risk factors for the recurrence of infection or the onset of new disease with NTM following lung transplantation are immunosuppression and the development of structural lung disease over time secondary to bronchiolitis obliterans 
syndrome..$^{110,111}$

The risk of developing NTM is also strongly associated with advanced age and the presence of comorbidities. This is believed to contribute to the recent increase in cases in the US given the aging of the population in the US, Canada and parts of Europe. ${ }^{112,113}$ Additionally, nonsmoker elderly females with a slender body along with characteristic features such as scoliosis, pectus defects, or mitral valve prolapse are more prone to pulmonary NTM as compared to the general population. ${ }^{114,115,116}$ In fact, NTM occurring in this population has been termed Lady Windermere syndrome, and has a characteristic presentation in the right middle lobe and/or lingua. Lady Windermere syndrome is believed to be associated with voluntary cough suppression that causes secretion accumulation creating an ideal place for growth of the NTM organisms. ${ }^{117}$ Also, the elderly are more likely to be complicated to treat given their increased likelihood to have comorbidities.

NTM infection is also more common in patients with specific behavioral risk factors such as tobacco use, alcohol use and poor nutrition. ${ }^{118}$ Despite well-defined risk groups, patients present with NTM infection without obvious risk factors or the present of underlying disease. ${ }^{119}$

Perhaps the most important host characteristic associated with NTM infection is HIV/AIDS. Given that HIV is still commonly diagnosed in 
the US during late stages of the disease, the presentation of NTM in an atypical host sometimes leads to an HIVIAIDS diagnosis. ${ }^{120}$ In fact, the presence of NTM is considered by many experts to be an AIDS defining illness, which is characterized by the presence of less than 200 CD4 Tcells that is often discovered following the diagnosis of opportunistic infections, which often includes NTM infection. ${ }^{121}$

HIVIAIDS represents one of the most important risk factors for NTM disease in patients of all ages including older populations. According to the CDC, people aged 55 and older accounted for an estimated $24 \%$ of the 1.2 million people living with HIV infection in the US in 2012.

The prevalence of HIVIAIDS in older populations can be attributed to several factors. First, the effectiveness of HAART (Highly Active AntiRetroviral Therapy) in extending life spans of those infected with the virus early during the HIVIAIDS epidemic have allowed HIV patients to age. ${ }^{123}$ HAART has extended lifespans well beyond expectation allowing many to age into retirement with a disease that was once considered a death sentence. The majority of this population is made up of the more traditional at risk population of HIV positive adults including those who acquired the disease through homosexual contact and IV drug use.

Newly diagnosed cases within this same age cohort have also increased in recent years. An estimated $27 \%$ of patients over 50 years of age accounted for new diagnoses of AIDS in 2013. The diagnosis of 
AIDS usually follows the identification of AIDS defining opportunistic infections, which often includes a diagnosis of NTM disease. In older populations, the CDC notes that late diagnoses are more common as compared to younger populations. ${ }^{124}$ Among the unique risk factors for this age group is a statistically significant increase of transmission through heterosexual contact, which has specifically impacted the incidence of HIVIAIDS in females over the age of 50 . Given the low perceived risk of heterosexual contact in this population, older adults are less likely to take HIVIAIDS prevention measures. ${ }^{125}$ The CDC notes that clinicians treating this population are less likely to suspect HIVIAIDS despite the presence of clinical symptomology and older patients are less willing to share with their care providers HIVIAIDS risk behaviors. ${ }^{126}$ Another important factor in the prevalence of disease in the older populations is the estimated $80 \%$ increase in erectile dysfunction treatment prescriptions in recent years. ${ }^{127,128}$

Another important factor is HIVIAIDS transmission in older populations, some scholars suggest that the aged are more likely to seroconvert than their younger counterparts if exposed to the disease. Among the explanations for this, age-related hormone production levels decrease in the aged, resulting in the thinning of the vaginal and anal mucosa. As a result, the likelihood of tearing during sexual contact increases which allows a point of entry for the virus. Also, age-related immunity function declines which leaves older adults more vulnerable to 
infection if exposed. ${ }^{129}$

\section{Transmission}

NTM species are environmentally ubiquitous organisms and disease transmission is believed to occur primarily from the environment. Having been isolated in various studies in soil and water sources, understanding the factors contributing to the transmission of disease to some hosts and not others remains an area of study. Emerging research has demonstrated that NTM can be transmitted person to person. Also, the relationship between humans and animals, and vice versa, is an important area of future research. Finally, the food supply, primarily fish living in water contaminated with NTM is another possible source of transmission.

Reports from different parts of the world have identified various species of NTM in environmental sources, including soil, dirt, household

water supplies, public water supplies, workplaces, and hospitals. ${ }^{130,131}$ In a review surveying data from several Middle Eastern countries, NTM was found in 619 environmental samples collected in Iran, Iraq, Turkey and Saudi Arabia and the most common species noted in these isolates was M. fortuitum. ${ }^{132}$ Several studies have also identified the presence of NTM in soil and water samples. Soil samples collected from a forest located in Finland identified the presence of NTM in $100 \%$ of the 47 samples. ${ }^{133} \mathrm{~A}$ Japanese study tested a variety of environmental samples and found the presence of NTM in $100 \%$ of 5 dirt samples, $100 \%$ of ditch mud samples, 
$77 \%$ of 22 house dust samples, and one of two river water samples. ${ }^{134}$

Water as a source of environmental NTM is well described in a variety of studies collected from several different water sources. An analysis of brook water collected from sources in rural Finland found that NTM was present in $100 \%$ of 53 samples. ${ }^{135}$ In an Australian study, the presence of MAC was identified by PCR in two water distribution pipelines. Later sampling during the summer months noted that the concentration of the organism significantly increased, which implies the organism multiplied while in the pipeline and became more infectious. ${ }^{136}$ In a study evaluating patients with MAC, NTM was identified in the home water samples from 10 patients. ${ }^{137}$

In a 2013 doctoral dissertation, the relationship between NTM isolated from a municipal water source and human infection was evaluated. In this study, water samples were taken from the municipal water supply in Queensland, Australia and compared with clinical samples collected from the same area. NTM was isolated in more than $40 \%$ of water samples during summer months and $82 \%$ of samples collected during winter months. The NTM species discovered included both species that are considered pathogenic to humans as well as nonpathogenic NTM. M. abscessus was not identified in water samples collected in this study. It is important to note that this particular NTM species is rarely isolated in water, as opposed to M. kansassi, which is so strongly associated with water, it has been termed the "tap water bacillus." Further investigation 
was conducted in the homes of 20 patients with a diagnosis of NTM in which samples collected from showerheads were compared. A total of 7 (35\%) patients were diagnosed with the same species of NTM that was identified in the showerheads of their home. This study also compared nosocomial, or hospital acquired, and community associated infections caused by $M$. fortuitum to the samples identified in the municipal water samples through strain analysis. Similarities were founds which implies contamination within the hospital water supply. Strain similarities were not found in community associated infections caused by $M$. fortuitum, indicating that water was likely not the mode of transmission in these cases. ${ }^{138}$ See below for comparison data of NTM species identified in this study in water samples and clinical isolates.

Table 6: Pathogens Collected from Water and Clinical Samples

\begin{tabular}{|c|c|c|}
\hline \multicolumn{2}{|c|}{ Pathogens Identified in Water } & Pathogens Collected \\
\hline M. gordonae & M. Ientiflavum & M. Ientiflavum \\
\hline M. szulgai & M. fortuitum & M. fortuitum \\
\hline M. simiae & M. kansasii & M. kansasii \\
\hline M. terrae & M. flavescens & M. abscessus \\
\hline M. chelonae & M. interjectum & \\
\hline M. intracellulare & M. mucogenicum & \\
\hline M. avium complex & & \\
\hline
\end{tabular}

Water from home and public water sources has been evaluated. In a study of 31 patients from the US and Canada with NTM disease due to 
MAC, M. abscessus, and M. xenopi, the species identified in the patient was also isolated in their home in $46 \%$ of cases. ${ }^{139}$ In a Saudi study, NTM was isolated from the mouthwash of $44(26.3 \%)$ subjects that used tap water versus $10(33 \%)$ subjects that used sterile water. ${ }^{140}$ In a review, the authors emphasized the importance of intentionally designing engineered water systems based on quantitative microbial risk assessment. This recommendation came following a discussion of numerous studies isolating NTM, legionellae and Pseudomonas aeruginosa from public water sources. ${ }^{141}$ Also, research has evaluated the possibility of transmission through aerosolized water. Theoretical sources of aerosolized NTM include primarily showerheads and hot tubs. A study examining household water and showerheads of patients with NTM pulmonary disease identified matched environmental and human strains of M. abscessus, M. lentiflavum, and M. kansasii in 20 patients $^{142}$ In a study evaluating 18 public hot tubs and warm water therapy pools, the presence of NTM in was found in 13 sites $(72 \%)$. Proper water care using chlorine or bromine sanitation significantly lowered the concentration of NTM identified in this study. ${ }^{143}$

NTM infection from food sources is another possible mode of transmission for which few data exist. One recent study suggests that NTM might be transmitted from freshwater fish and fish products following the review of 92 tissue samples obtained from ponds, retail fresh, frozen fish and smoked fish products. In this study, nearly $12 \%$ of samples 
evaluated tested positive for pathogenic NTM. ${ }^{144}$

Although NTM was previously believed to only be transmitted from environmental sources, emerging data suggests that human-to-human transmission occurs in patients with cystic fibrosis. A 2011 report in which 168 isolates collected from 31 cystic fibrosis patients with NTM infection due to $M$. abscessus was tested using whole genome sequencing and drug susceptibility testing revealed the frequent transmission of multidrug resistant NTM between patients. ${ }^{145}$

Despite these reports, the transmission patterns of NTM are not well described. Transmission from environmental sources seems to occur from a myriad of soil and water sources that surrounds communities, and, in some cases, lives in our homes while emerging data indicate that NTM may even be transmitted from food sources. The factors influencing the transmission of disease from environmental pathogens remain

unknown. ${ }^{146}$ Further, the transmission of NTM disease from animals to human, humans to animals and humans to humans is a critical area of future research. Improving the understanding of transmission pathways is critical to crafting a more effective response to this emerging public health problem.

\section{Diagnosis}

The diagnosis of NTM infection involves the correlation of clinical signs and symptoms with radiological and microbiological findings. Given 
its similar appearance to TB, NTM diagnostics can be especially challenging as the two diseases may appear identical in disease presentation. Also like TB, the diagnostic process consists of several important components and multiple layers.

The microbiological studies process begins with a smear stain, which determines whether the specimen is a mycobacterium species. Common smear staining techniques include fluorescence microscopy and Ziehl-Neelsen. Given the differing sensitivity and specificity of these staining procedures for various mycobacterium species, a combination of smear techniques may be used. ${ }^{147}$ Once the specimens are determined to be acid fast bacilli, specimens are prepared for isolation. The first step in this process involves the use of $\mathrm{N}$-acetyl-L-cysteine-sodium hydroxide (NALC/NaOH), which serves as a decontaminant that prevents the growth of other bacteria. ${ }^{148}$

When smears are followed by cultures, the positive identification of NTM depends of the growth rate of the organism. Most NTM strains grow within two to three weeks with the exception of rapidly growing mycobacteria species $M$. abscessus, $M$. fortuitum, $M$. chelonae, and $M$. massiliense, which may grow within 7 days. ${ }^{149}$

Recently developed methods may also be used for a quicker result. High-performance liquid chromatography (HPLC) is a molecular method that involves the use of species specific probes. ${ }^{150}$ HPLC recognizes 
mycobacteria according to variations in mycolic acids, the long-chain fatty acids resided in the cell wall of mycobacteria. ${ }^{151}$ The major advantage of HPLC is rapid species identification. ${ }^{152}$ However, molecular DNA probes are available for identifying a select few NTM species including MAC, $M$. gordonae, and M. kansasii. Therefore, NTM infection due to other causes is not diagnosable using this method. The HPLC method is also more costly, requires reliable laboratory infrastructure and well trained technicians to carry out the test, which may limit its availability in some care settings. ${ }^{153,154} \mathrm{PCR}$ restriction fragment length polymorphism analysis is another molecular technique for identifying mycobacteria on account of differences in restriction fragments of the $65 \mathrm{kD}$ heat-shock protein. Sequence analysis of the rpoB gene and $16 S$ ribosomal RNA has been expanded recently as another method for speciation of NTM. ${ }^{155,156}$

Significant improvements in the laboratory methods that identify the presence of mycobacteria have occurred over the last two decades. Also, species identification has allowed the opportunity to further expand the clinical and epidemiologic database regarding NTM. ${ }^{157}$ Despite recent advances, diagnostics remains challenging for several reasons. First, due to the ubiquitous presence of NTM species in the environment, the likelihood of contamination is high and can occur before, during or after clinical samples are collected. Many possible scenarios exist for contamination. For example, sputum samples may be contaminated when traveling through the mouth during collection. ${ }^{158}$ Following arrival to the 
laboratory, samples may be contaminated by equipment. The contamination of a fibrotic bronchoscope suction channel has been reported as the cause of several positive cultures for M. chelonae. ${ }^{159}$ Given that many mycobacterium species live in water, it has been reported that liquids used during the culture process exist as another possible source of contamination. ${ }^{160}$ For these reasons, the reliance on microbiological findings must be carefully considered alongside clinical and radiological. ${ }^{161,162}$

The diagnosis of NTM disease is defined by the positive identification of a pathogenic NTM species in a clinical isolate in addition to clinical signs and symptoms of disease. In a statement published by the American Thoracic Society/ Infectious Diseases Society of America (ATS/IDSA) is the official definition of pulmonary NTM as outlined in the table below. ${ }^{164}$

Table 7: ATS/IDSA Official Definition of pulmonary NTM

\section{ATS/IDSA Criteria for pulmonary NTM}

\begin{tabular}{|c|l|}
\hline 1 & $\begin{array}{l}\text { Chest radiograph showing opacities, nodular or cavitary, or a high- } \\
\text { resolution computed tomography (HRCT) scan that shows multifocal } \\
\text { bronchiectasis with multiple small nodules; and }\end{array}$ \\
\hline 2 & $\begin{array}{l}\text { Three or more sputum specimens positive for acid-fast bacilli (AFB); } \\
\text { and }\end{array}$ \\
\hline 3 & The exclusion of other causes including TB. \\
\hline
\end{tabular}

The panel of experts emphasized that these criteria were designed 
for the unique infectious processes of MAC, $M$. kansasii, and $M$. abscessus, noting that ATS/IDSA criteria cannot be applied to other species of NTM because there is not enough data to confirm a similar infectious process for all species of NTM. ${ }^{165}$

Following the diagnosis of NTM infection and the identification of NTM species, the next critical step in NTM infection management is DST. For NTM, laboratory isolates are tested against common antimicrobial agents in order to optimize therapy. This is particularly important because most NTM strains are usually resistant to agents used to treat TB, leaving fewer options for treatment as compared to many other diseases. Also, many preferred agents such as clarithromycin may not be effective in some strains. ${ }^{167}$ Current ATS/IDSA guidelines recommend drug susceptibility tests for MAC against macrolide antibiotic class, M. kansasii against rifampin, and rapid growing mycobacteria against various antibiotic classes including newer macrolides and quinolones, doxycycline and minocycline, and sulfonamides. ${ }^{168}$

\section{Treatment}

The treatment of NTM is long-term and complex consisting of bacteriologic surveillance, monitoring for drug toxicity, and managing comorbidities. Also, with the absence of randomized clinical trials published in peer-reviewed literature, current guidelines for treatment are based largely on expert opinion using what little data are available. Guidelines vary depending on site of infection, host characteristics, and 
NTM species. Therapy duration is also highly variable, dependent largely on repetitive negative cultures for a period of time, usually one year. Given these long treatment courses, monitoring and managing the sometimes harmful side effects associated with long-term antibiotic use is an important component of NTM management. Further, because NTM patients tend to have underlying diseases coupled with advanced age, treatment can be complicated with providers having to manage drug therapy and side effects alongside comorbid conditions and associated therapy. Finally, all of this requires strong patient compliance over a long treatment course.

An example of NTM therapy as recommended by the ATS/IDSA for NTM species MAC for patients who are not HIV positive with pulmonary infection may include clarithromycin (1000 mg three times per week) or azithromycin (500 mg three times per week); and rifampin (600 mg three times per week) or rifabutin (300 mg three times per week); and ethambutol ( $25 \mathrm{mg} / \mathrm{kg}$ three times per week). For patients with fibrocavitary MAC lung disease or severe nodular or bronchiectatic disease, the ATS/IDSA guidelines recommend clarithromycin (500 to 1000 mg daily) or azithromycin (250 mg daily); and rifampin (600 mg daily) or rifabutin (150 to $300 \mathrm{mg}$ daily); and ethambutol (15 mg/kg daily). In some cases, streptomycin or amikacin (both 10 to $15 \mathrm{mg} / \mathrm{kg}$ three times per week) may be added as a fourth agent for the first eight weeks. A lower dose (6 to $8 \mathrm{mg} / \mathrm{kg}$ two to three times weekly) may be appropriate for 
patients who are older than 50 years of age, whose weight is $<50 \mathrm{~kg}$, or who require parenteral therapy for longer than two to three months. For the same patient population with M. kansasii, the second most common NTM organism, ATS guidelines recommend combination therapy consisting of isoniazid (300 mg daily), rifampin (600 mg daily), ethambutol (15 mg/kg per day) in the same patient population. Therapy duration is generally one year following the first negative culture result assuming subsequent results are also negative. ${ }^{169}$

The presence of comorbid conditions also contributes to potential treatment complications for NTM disease. As many older Americans maintain regimens of maintenance medications, prolonged concurrent therapy can present clinicians with many treatment challenges. For this reason, NTM treatment must be chosen carefully in order to avoid drugdrug interactions with existing and necessary concurrent medication. Antibiotic classes commonly used to treat NTM cause interaction with drugs that interact with cytochrome P-450 (CYP), which includes drugs commonly used to treat a myriad of disorders including anxiety, psychiatric disorders, seizure disorders, hyperlipidemia, hypertriglyceridemia, atrial fibrillation, and bleeding disorders. ${ }^{170}$ In other patient populations such as patients that are HIV positive, patients with extra pulmonary infection sites or disseminated disease, or patients with documented resistance following DST or previous treatment failures, other recommendations using less proven treatment regimens may be used. 
In select cases of NTM infection, surgical resection may be a consideration. Indications for surgery have not been uniformly defined, but are most commonly associated with medication intolerance, drug resistance, localized cavitation, recurrent or massive hemoptysis, and the presence of a destroyed lung. The safety of lung resection for NTM lung disease, particularly thoracoscopic right middle lobe lobectomy and lingulectomy, has been reported as favorable in carefully selected patient populations and under the care of experienced surgeons. Data have shown that surgery provides improved microbiologic response for refractory organisms such as $M$. abscessus as compared to medication regimens alone. ${ }^{171,172,173}$

\section{Epidemiology}

Infections caused by NTM are an increasingly important cause of morbidity and mortality. Infection is the result of the interaction between exposure load and host characteristics including underlying diseases, age, behaviors, and genetic predispositions. Virtually everyone is routinely exposed to NTM, although most do not develop clinical signs of infection. The factors predisposing one to infection are not well understood. ${ }^{174}$ United States population-based data indicate that the number of deaths from NTM disease is growing. During the years 1999 through 2010, NTM disease was reported as an immediate cause of death in 2,990 people in the United States with a combined overall mean age-adjusted mortality rate of 0.1 per 100,000 person/years. Persons aged 55 years and older, 
women, those living in Hawaii and Louisiana, and those of non-Hispanic, white ethnicity had higher mortality rates. In fact, some evidence suggests that NTM is a more common cause of death in the US than TB. ${ }^{175}$ The majority of NTM deaths were reported in the hospital setting. ${ }^{176}$ Additionally, there is a strong association between age and NTM mortality, which was found to be significantly higher in patients older than 65 years. In addition to the presence of comorbidities common in this population, advanced age itself was determined to be a strong predictor of mortality. ${ }^{177,178}$ Morbidity can also be significant for patients with NTM infection. A national cohort study found that respiratory failure is more common in patients with NTM after adjusting for potential confounders such as age and the presence of comorbidities. ${ }^{179}$

The epidemiology of NTM is vastly different from TB, and has changed significantly in recent years. First, NTM is becoming increasingly common in wealthy industrialized countries, while respective TB rates are on the decline. ${ }^{180}$ However, NTM is a non-reportable disease in the US and its epidemiology is not well described in the literature or by other sources of disease surveillance, resulting in infection rates being difficult to piece together. The prevalence of disease in North America was reported at $1.6-1.8$ per 100,000 in the 1980 s whereas recent reports record a prevalence of 14.1 per $100,000 .^{181}$ Current reports indicate that NTM exceeds TB infection rates in the USA according to data from Oregon found the total age-adjusted prevalence of NTM was reported at 
8.6 per 100,000 cases in the $2005-2006$. This number increased to 20.4 per 100,000 in adults 50 years of age and older. The median age was 66 years and $59 \%$ were females. ${ }^{182}$ In a combined report of four other regions in 2010 , the mean annual prevalence was 5.5 per 100,000 , ranging from 1.7 per 100,000 in Southern Colorado to 6.7 per 100,000 in Southern California. ${ }^{183}$ According to findings from a national Medicare claims data study, the annual prevalence of NTM in the population older than 65 years old significantly increased from 20 cases per 100,000 persons in 1997 to 47 cases per 100,000 persons in 2007 . The study also found interesting demographic features, including that Caucasians accounted for $90 \%$ of cases followed by Asians/Pacific Islanders and African-Americans. ${ }^{184,185}$

A number of explanations exist for these trends. First, North America and Europe demonstrate a dramatic decline in TB rates given successful public health interventions coupled with the development of anti-TB medication over the last century. Also, the recognition of NTM along with the ongoing identification of more NTM species have allowed clinicians to improve diagnostic methods so that NTM cases are not misdiagnosed and treated as TB. Some of this technology is not available or affordable in developing countries, resulting in NTM not only being misdiagnosed and treated, but also facilitating resistance to anti-TB drugs characterizing MDR and XDR-TB. Another important contributor to the rise in NTM infection in North America and Europe is a population aging. 
Environmental factors are also important as warm and humid climates have been associated with higher rates of infection.

The population composition of North America and many parts of Europe demonstrate population aging coupled with overall population shrinking. In the US, population composition is uniquely impacted by the baby boomer generational cohort. The baby boom is characterized by the post World War II bump in birth rates that began in the middle of 1946 and continued into 1960. This noticeable increase in births exists alongside a decline in births during subsequent years, resulting in the baby boomers commanding a significant proportion of the population. In 2010, the elderly in the US was estimated to be approximately 40.2 million. Given that baby boomers are just now entering retirement, the number of elderly Americans is expected to grow in coming decades. Further, population composition projections demonstrate this trend will remain as the baby boomer cohort enters the very elderly years. ${ }^{186}$ Projections from the US Census Bureau show population structure challenges associated with the baby boomer generational cohort marks only the beginning of elderly Americans making up a significant proportion of the population. ${ }^{187}$ As the generation after the baby boomers enters retirement years, the number of elderly Americans is expected to increase to 88.5 million by $2050{ }^{188}$ See the figures below for projections from the US Census Bureau that demonstrate the impact of the baby boomer generational cohort relative to other generations beginning in 2012, 2035 and $2060 .^{189}$ 


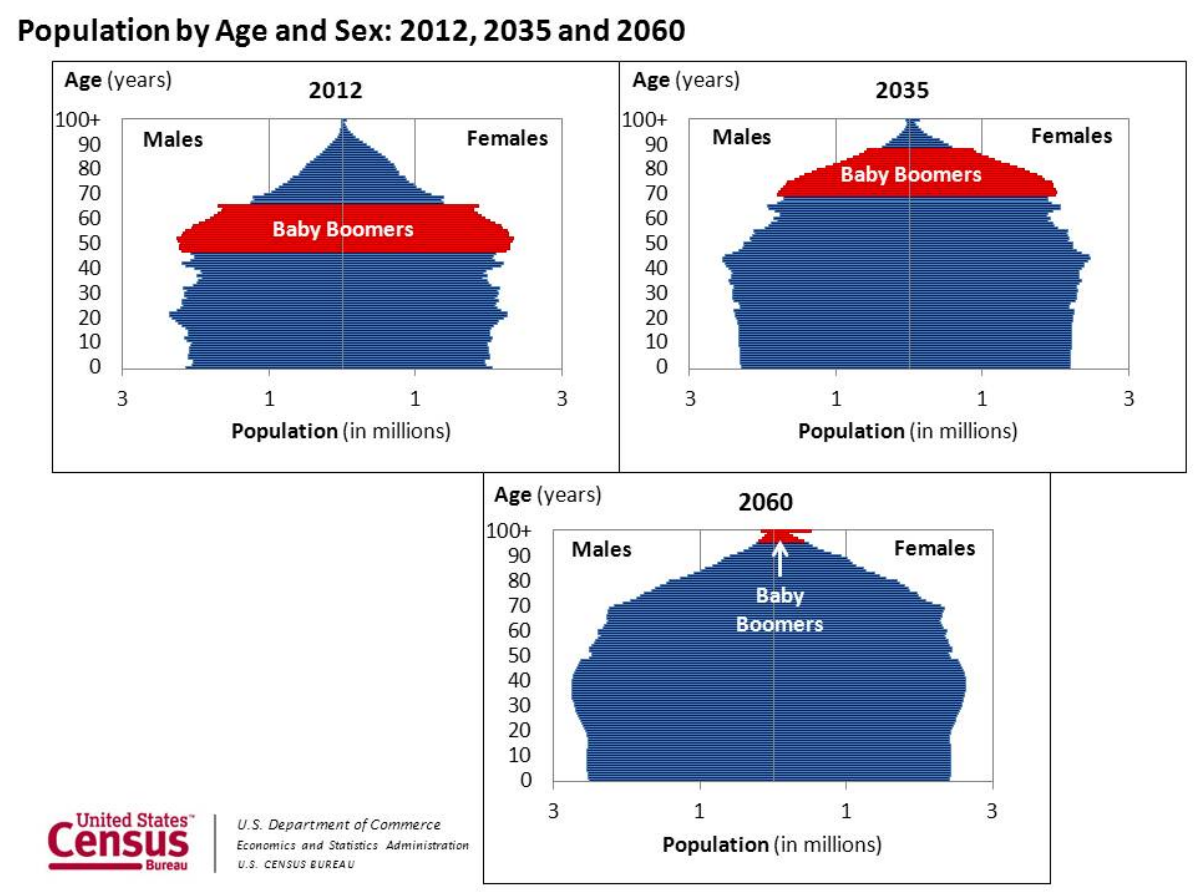

Figure 4: US population by age and sex in 2012 and projections

Given that many entitlement programs in the developed world are in place relying on the support of younger citizens, population aging and shrinking has resulted in deficits and shortfalls. In the US, the future of Medicare and social security have been an important political debate for several election cycles as policy makers have expressed concern with funding these programs in the future using conventional methods. Also, the high utilization of healthcare resources by the aging population has also been an important cost driver for healthcare in the US that is only expected to worsen as the baby boomers continue to age. Therefore, population aging has had many important implications for the funding of public programs as well as managing the cost of many illnesses associated with the elderly including NTM in the US and in other 
developed countries. ${ }^{191,192}$

NTM prevalence differs by geographic region due to specific environmental factors linked to climate, water, and soil exposure. Given the most common sources of NTM species are water and soil, swampy, humid southeastern states in the US have more cases of disease than the dry west or the cold north. A report of pulmonary NTM identified 55 counties in 8 states with a particularly high risk of infection, which included parts of California, New York, Florida, Hawaii, Louisiana, Oklahoma, Wisconsin, and Pennsylvania. A map of state reported variations in NTM infection are illustrated blow. ${ }^{193}$

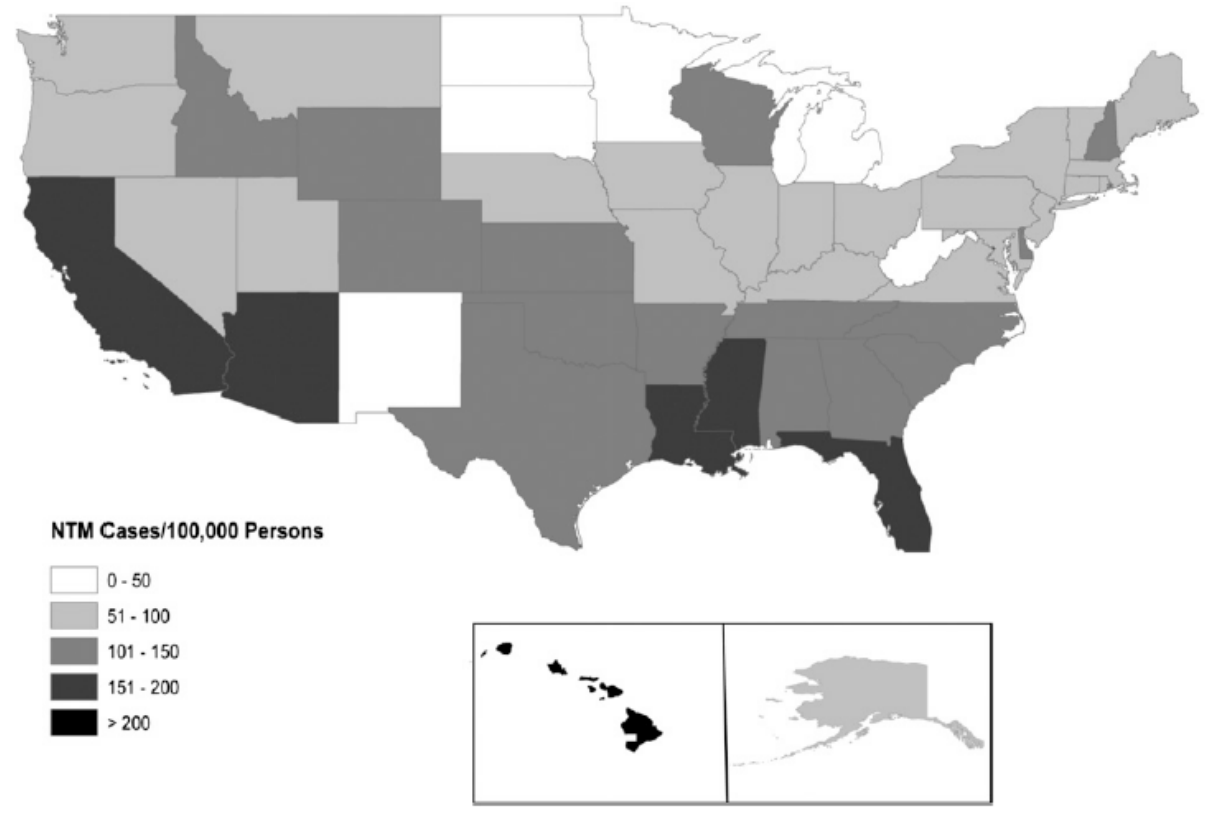

Figure 5: NTM rates by state in the US

NTM is an important infectious disease that represents a new public health challenge. Given its similarity to TB both in both presentation and microbiology, NTM was only recently recognized as a 
human pathogen. ${ }^{194,195}$ Diseases caused by NTM generally have prolonged treatment in order to achieve a complete cure. Therefore the prevalence is far greater than the incidence. While efforts in treatment and surveillance of TB have resulted in a decrease in the incidence in recent years, the incidence of NTM is increasing which has classified NTM as an emerging public health problem in the US and the world. ${ }^{196,197,198}$ In a national cohort study, populations underwent screening for the NTM species M. intracellulare during the years 1971-1972 and 1999-2000. In the earlier cohort, a total of 1,490 participants were evaluated and 7,384 were included in the later cohort. This study found that one in nine participants in the 1970s tested positive as compared to one in six in 1999 and 2000. The authors conclude these findings are consistent with the overall rising rates of NTM in the US. ${ }^{199}$ According to a study conducted in Oregon, NTM rates were in excess of TB in the population sampled. Given that Oregon has TB rates comparable to fourteen other states as well as demographic similarities with thirty-five other states, the authors suggest that NTM is likely more prevalent than TB in several states in the US. $^{200}$

Recent surveillance data was presented at the 2016 IDSA meeting in New Orleans, LA. In this study, data was retrieved from the National Center for Health Statistics between the years 1999 and 2014 for cases in which NTM was noted as a contributing or underlying cause of death. This database includes causes of death as recorded on death certificates 
along with demographic data of the deceased from all fifty states in the US. Demographic data was collected including age, race, gender and comorbid conditions. This study established the mortality burden of NTM at 2.3 per 1,000,000 persons over the past 15 years. This study also confirmed established demographic features of the disease, noting that mortality was significantly higher among older white women. Also, comorbid conditions most strongly associated with mortality were structural lung diseases such as cystic fibrosis, bronchiectasis and pneumoconiosis. Further, this study found that HIV status was the only non-pulmonary comorbidity that was found to be a predictor of mortality; however, data from this study noted that HIV co-infection rates in patients dying with NTM infection declined during the fifteen year study period as NTM rates in patients without HIV infection has increased. The purpose of this study was to provide national surveillance data for NTM mortality rates given that this disease is not reportable and the burden of mortality is unknown. ${ }^{201}$ 


\section{CHAPTER 2: STATEMENT OF THE PROBLEM AND LITERATURE REVIEW}

Mycobacterial infections are a source of significant economic burden in the US and the world. The cost burden of TB and NTM are multifaceted, including complex direct medical costs which include not only managing the infection and related therapies and procedures, but also minimizing the impact of common side effects, identifying and preventing potentially dangerous adverse reactions, managing comorbid conditions as they relate to therapy, and minimizing the cost of treatment failures and other therapy challenges. Given that health care costs have been key factors in national economies worldwide, controlling health care costs has become a priority for essentially all countries in the world, and is an important area of research.

\section{Mycobacterium tuberculosis}

Mycobacterium tuberculosis (TB) represents a worldwide public health challenge with corresponding cost implications. In the developed world, TB was once a nearly eradicated disease that has resurfaced secondary a number of factors. Among these are immune suppression as a result to the HIVIAIDS epidemic, diabetes mellitus, or immune 
suppression therapies, immigration, and the development of drug resistance. ${ }^{202}$ In the underdeveloped world, TB has emerged with an often disproportionately high rate of HIVIAIDS, malnutrition and other poverty related causes of immune suppression, coupled with resource constrained health care delivery systems. In many of these countries, national TB programs have been established to deliver TB related treatment at no direct cost to the patient. However, the reality of many TB programs in resource-constrained countries is characterized by a failure of to meet public demand, limited access to drug therapies, and a reliance on outdated or incomplete laboratory methods. Also, indirect costs associated with TB disease such as food and travel can result in further impoverishment for the very poor. This situation is often exacerbated by the loss of wages secondary to the disease process and the time investment necessary to seek treatment. In these cases, adding the cost of direct medical care can be potentially devastating to many vulnerable populations. ${ }^{203}$

\section{TB in the Underdeveloped World}

The prevalence of TB is disproportionately high in developing countries located primarily in Asia and Africa. Given the strong association between TB and poverty, the risk of driving vulnerable populations into further poverty as a result of TB infection is important with significant implications for long-term quality of life. Several studies have examined cost as a means of measuring the impact of TB on individuals 
and their families as well as in the context of national resource planning. In the current body of medical literature, the financial burden of TB has been measured from a variety of perspectives. Also, cost data are widely available in the context of national and international policy, resource planning, technology assessment as well as the measurement direct and indirect treatment cost relevant to patients and their families. The conclusion of the majority of these studies is not encouraging, noting that TB can be particularly devastating for patients, their families and/or caregivers, as well as threatens the fragile healthcare delivery systems in resource scarce countries.

\section{Program Delivery Costs}

The TB delivery system in most countries is complex resulting in many challenges in measuring cost. For example, in countries that theoretically provide TB care at no direct cost to the patient, free services may not be available due to resource constraints, may not be offered to patients due to unavailability or corruption, or indirect expenses or lost wages may not be offset with vouchers or other expense reimbursement and wage replacement programs. Only one of these factors being present within many populations can be financially devastating.

The financial impact of TB disease is heterogeneous in the developing world and dependent upon many important factors. A systematic review of 49 studies from a variety of low resource countries located in Africa, Asia, and Latin America found large variations in costs 
often within the same care setting and among different care settings, but most of which were significant relative to middle and low income populations. Despite TB related treatment having been delivered at no direct cost to the patient in some care settings included in this analysis, this study found that patients were sometimes not offered free services despite their availability, patients received services from private sector providers that were not included in national TB programs, or patients were responsible for ancillary medical costs such as adjunct therapy for TB drugs or routine hospitalization. In addition to direct medical costs, this systematic review also found significant indirect costs, which included transportation to treatment facilities and food costs during travel. This study emphasized that travel and food vouchers could be an important part of a successful TB program for low-income populations. Perhaps the most critical factor driving cost identified across studies was the ability to work and earn wages. Both treatment obligations and the physical impact of illness undermined the ability to work and earn normal wages placed patients at the highest risk of financial vulnerability. The average cost breakdown across studies was as follows: $20 \%$ direct medical care, $20 \%$ indirect medical care, and $60 \%$ lost wages. ${ }^{204}$

Perhaps one of the least described variables is the impact of illness on the ability to earn wages. As the previous study notes, lost wages are one of the most important cost factors related to the impact on the patient. Lost wages are driven by several inputs: time away from work seeking 
treatment including hospitalization, the impact of the disease process on the ability to work, and the impact of wage earning on family members and caregivers. Despite this, lost wages in low to middle resource countries is rarely and often incompletely measured. Based on the results from a systematic review, it is believed that lost wages accounts for as much as $60 \%$ of total cost experienced by patients and their families. ${ }^{205}$ For this reason, the economic impact of TB was noted as a major concern following an interview of TB patients at an urban clinic in Bombay, India. This study sought to understand the perceptions of TB and the impact of a TB diagnosis and subsequent treatment on the daily lives of patients. Following intense interviews of 16 respondents, men expressed financial concerns including: loss of wages, reduced capacity for work, poor job performance, and absenteeism from work. It is important to note that the surveyed men were primarily self-employed so they did not express concerns about losing their job, but women were more likely to be employed in this sample and did express that concern. Women also feared rejection by their husbands while single women feared that their illness may reduce their chances of finding a husband, which also has important financial implications. ${ }^{206}$ Another focus group based study conducted in rural India of 304 TB patients found that an average of 83 work days were lost following a diagnosis of TB. ${ }^{207}$ Additionally, the disease process itself has a significant physical toll, which has important implications for one's ability to work while infected with TB. In fact, body 
composition changes, or wasting, is one of the most notable features of active disease and is attributable to a number of factors which may include a loss of appetite as well as metabolic changes secondary to inflammatory and immune responses. ${ }^{208,209,210,211}$ The relationship between wasting and physical impairment is well described, although the economic implications of this relationship are not that well understood. ${ }^{212,213}$ However, it is noteworthy that emerging research has identified important risk factors for wasting including low socio-economic status and risk behaviors associated with low socio-economic status, indicating the impact of disease is more pronounced in vulnerable populations. ${ }^{214}$ Wasting is also an important risk factor for mortality, which also has important economic implications. ${ }^{215}$ A study indicated that over 500,000 disability-adjusted life years are lost due to illness and mortality secondary to TB each year in the Philippines, with an economic cost equivalent to $\$ 145$ million. ${ }^{216}$

Several inquiries into the national TB program in China illustrate many of the realities of TB care as found in developing countries. China provides TB related treatment to patients free of charge as the result of a national program developed and subsidized by the World Bank following a resurgence in TB rates in the 1990s. This national program consists of a local delivery system on the county level and provides 6 months of TB therapy for treatment naïve patients and 8 months for treatment experienced patients along with a sputum smear test, and chest x-ray at a local treatment center. Initial reports evaluating the national TB program 
in China, early in its implementation phase, boasted positive results, reporting $98.4 \%$ adherence in a population of over 55,000 patients. ${ }^{217}$ However, subsequent reports have noted that TB treatment providers recommend treatment courses beyond what is provided by the national TB program, along with additional laboratory methods, imaging studies and ancillary treatment such as liver preserving therapies at an additional cost to patients. ${ }^{218}$ A systematic review measuring direct medical costs incurred by TB patients in China sought to trace the relationship between cost and adherence and completion of TB related treatment. Data extrapolated from published studies and data from Chinese and international organizations found that low-income patients pay within an average range of $42 \%$ to $119 \%$ of their annual household income for TB treatment in direct medical care costs. Also, $73 \%$ of TB patients admitted to having interrupted or suspended their treatment, citing cost as the most common reason. This study found that despite the Chinese policy of TB care delivered at no cost to patients, TB patients and their families are paying significantly and often failing to complete their treatment as a result of direct medical cost. ${ }^{219}$

Another systematic review focusing on countries in sub-Saharan Africa pulled direct and indirect cost data published in thirty studies from the following countries: Zambia, Nigeria, Kenya, Malawi, Ethiopia, Uganda, South Africa, Sierra Leone, Gambia, Botswana and Tanzania. This review included a descriptive classification of costs including those 
incurred in the private sector prior to patients being transferred to the public sector for treatment through free national TB programs. In addition to private sector provider fees, additional direct costs included health insurance premiums, prepayment fees, and tipping of medical providers as practiced in Uganda. Diagnostic, treatment and hospitalization costs were reported and sometimes included admission fees, culture and imaging costs, treatment costs, and needle and syringe costs. Another descriptive cost item noted in five studies were costs associated with visits to traditional healers, and four studies noted the cost of food including special therapeutic food. Of course, travel costs were also noted as well as time costs, which equated to lost work time. Not all studies reported every cost category, but in many cases the presence of only one of these costs could easily exceed the resources of the poor and some middleincome patients. For example, the cost of TB care reported in Malawi relative to monthly income ranged from $129 \%$ to $244 \%$ in the sample evaluated. More than one cost classification was present for TB patients in Zambia with $16 \%$ of monthly income spent on transportation costs, $66 \%$ spent on food costs, and a range of $10 \%$ to $132 \%$ having been allocated to treatment costs. In Zambia, female patients were reported to spend much more on TB treatment than male patients. In Ethiopia, $48 \%$ of monthly income was spent on treatment and $35 \%$ was spent on pretreatment. In addition to patient cost, eight studies noted direct and indirect costs incurred by caregivers or guardians. In twenty of the thirty 
studies, direct and indirect costs associated with TB were found to be catastrophic for those of average income while twenty five studies found costs to be catastrophic for those of low income as defined as the bottom $20 \%$. Therefore, a diagnosis of TB in sub-Saharan Africa can either cause or exacerbate poverty as well as impact the willingness for patients to seek care for TB. The delay of treatment has additional implications, which can result in the presentation of more advanced disease and facilitate the transmission of disease to others. ${ }^{220}$

Following a survey based study of national TB programs in Ghana, Vietnam and the Dominican Republic, direct and indirect costs incurred by patients were measured from a sample of TB patients who had undergone at least one month of therapy. This study found the economic impact of TB to be significant in care settings on three different continents. For example, between $27 \%$ and $70 \%$ of patients reported that they had stopped working as a result of their diagnosis. Also, between $5 \%$ and $37 \%$ sold property and between $17 \%$ and $47 \%$ borrowed money in order to pay for direct and indirect medical costs and account for lost wages. The total average costs incurred by patients in all care settings were equivalent to approximately 1 year of individual income. ${ }^{221}$

The impact of TB-related expenses on accessing and adhering to quality care has been addressed in several reports by the World Health Organization (WHO). In its report encouraging all states to adopt some form of universal access to healthcare resources, TB is noted as one 
particularly devastating disease that can result in financial hardship if care is accessed without financial support. ${ }^{222}$ Also, a subsequent report noting the potentially catastrophic financial costs TB affected patients and their families face in many care settings due to direct and indirect health care costs also emphasized the need for income replacement and social support in the event of illness. ${ }^{223}$

Given the consistent findings from individual studies and systematic reviews, the weaknesses of many national TB programs that fail to deliver quality and cost effective TB care has been widely documented. As a result, several studies have been published to facilitate improvements in the allocation of TB diagnostic and treatment resources.

A study of the national TB program in South Africa found insufficient resources being allocating in research and development despite South Africa having had the second largest incidence rate of TB in the world according to 2010 data. The epidemic in South Africa has been facilitated by the HIVIAIDS epidemic, which has disproportionately affected sub-Sahara African nations. According to the WHO, TB is the most common co-infection disease of HIVIAIDS and is the cause of death of one quarter of HIVIAIDS patients. ${ }^{224}$ Reports from sub-Saharan Africa reveal that TB is often undiagnosed in HIVIAIDS patients and death due to disseminated disease is not uncommon. ${ }^{225,226}$ South African TB prevention policy encourages the 1.5 million HIVIAIDS patients to receive first line drug isoniazid as part of active TB disease prevention therapy. 
When this figure is combined with the nearly half million cases of active TB annually, this equates to nearly 2 million treatment regimens. This study likewise found that South Africa is providing only a fraction of treatment demand due to resource constraints. ${ }^{227}$

Research and new drug development for new treatment regimens have been slow. The model has historically been to rely on higher resource countries to facilitate new drug discovery, but this model has not been effective for TB for several reasons. First, the global impact of TB has been outside of high resource countries resulting in the discovery of new drugs having a low impact on the settings and funding for new therapies. Also, high prevalence TB countries tend to be low to middle resource with public delivery systems in place resulting in a resourceconstrained public sector unable to pay market premiums for new therapies. For these reasons, TB research and development will not provide a suitable return on investment as would be expected by the private sector in high resource countries. Despite arguments that drug development should still occur due to a moral obligation, new drug development for TB has remained inadequate relative to global demand. As a result, policy makers are beginning to encourage a stronger investment in research and development in countries with a high burden of disease, offering a mathematical model demonstrating a suitable return on investment given the crippling disease burden in some countries. ${ }^{228}$ The table below provides a summary of costs of TB delivery systems. 
Table 8: Program delivery costs for budgets and patients

\begin{tabular}{|c|c|c|c|c|}
\hline Author(s) & Setting & Study Type & Study Size & Findings \\
\hline $\begin{array}{l}\text { Tanimura } \\
\text { et al. }\end{array}$ & $\begin{array}{l}\text { Africa, Asia, } \\
\text { and Latin } \\
\text { America }\end{array}$ & $\begin{array}{l}\text { Systematic } \\
\text { Review of } \\
\text { literature }\end{array}$ & 49 studies & $\begin{array}{l}20 \% \text { Direct, } 20 \% \\
\text { Indirect, } 60 \% \text { Lost } \\
\text { Wages }\end{array}$ \\
\hline Nair et al. & $\begin{array}{l}\text { Bombay, } \\
\text { India }\end{array}$ & Interview & $\begin{array}{l}16 \text { low } \\
\text { income } \\
\text { patients } \\
\text { with TB }\end{array}$ & $\begin{array}{l}44 \% \text { Male, majority } \\
\text { were self employed and } \\
\text { reported lost earning; } \\
66 \% \text { Female, reported } \\
\text { job loss as most were } \\
\text { employed and rejection } \\
\text { by husband or potential } \\
\text { mate }\end{array}$ \\
\hline $\begin{array}{l}\text { Rajeswari } \\
\text { et al. }\end{array}$ & Rural India & Survey & $\begin{array}{l}304 \\
\text { respondents }\end{array}$ & $\begin{array}{l}83 \text { average work days } \\
\text { lost }\end{array}$ \\
\hline $\begin{array}{l}\text { Praygod } \\
\text { et al. }\end{array}$ & Tanzania & $\begin{array}{l}\text { Cross- } \\
\text { sectional of } \\
\text { newly } \\
\text { diagnosed } \\
\text { TB patients } \\
\text { 2007-2008 }\end{array}$ & $\begin{array}{l}201 \\
\text { pulmonary } \\
\text { TB patients }\end{array}$ & $\begin{array}{l}\text { Female: } 37.8 \%(76) \\
\text { Male: } 62.2 \%(124) \\
\text { HIV +: } 51.7 \%(104) \\
\text { Wasting strongly } \\
\text { associated with risk } \\
\text { behaviors in vulnerable } \\
\text { low socio-economic } \\
\text { populations }\end{array}$ \\
\hline $\begin{array}{l}\text { Peabody } \\
\text { et al. }\end{array}$ & Philippines & $\begin{array}{l}\text { Surveys } \\
\text { and national } \\
\text { datasets }\end{array}$ & $\begin{array}{l}\text { Population } \\
\text { based data }\end{array}$ & $\begin{array}{l}\text { Wasting associated } \\
\text { with } \$ 145 \text { million in } \\
\text { costs and } 500,000 \\
\text { disability adjusted life } \\
\text { years }\end{array}$ \\
\hline Long et al. & China & $\begin{array}{l}\text { Systematic } \\
\text { Review of } \\
\text { literature }\end{array}$ & $\begin{array}{l}247 \text { studies, } \\
4 \text { national } \\
\text { survey } \\
\text { datasets }\end{array}$ & $\begin{array}{l}\text { Direct medical care } \\
42 \%-119 \% \text { of annual } \\
\text { income, } 73 \% \text { did not } \\
\text { finish treatment due to } \\
\text { cost }\end{array}$ \\
\hline $\begin{array}{l}\text { Barter et } \\
\text { al. }\end{array}$ & $\begin{array}{l}\text { Sub- } \\
\text { Saharan } \\
\text { Africa }\end{array}$ & $\begin{array}{l}\text { Systematic } \\
\text { Review of } \\
\text { literature }\end{array}$ & 30 studies & $\begin{array}{l}\text { Cost of treatment } \\
\text { was catastrophic for } \\
\text { average income in } 20 \\
\text { studies; bottom } 20 \% \\
\text { income in } 25 \text { studies }\end{array}$ \\
\hline $\begin{array}{l}\text { Mauch et } \\
\text { al. }\end{array}$ & $\begin{array}{l}\text { Ghana, } \\
\text { Vietnam, } \\
\text { and } \\
\text { Dominican } \\
\text { Republic }\end{array}$ & Survey & $\begin{array}{l}135 \\
\text { patients } \\
\text { with TB }\end{array}$ & $\begin{array}{l}27 \%-70 \% \text { stopped } \\
\text { working, } 5 \%-37 \% \\
\text { sold property, } 17 \% \text { - } \\
47 \% \text { borrowed } \\
\text { money, average cost } \\
\text { was equivalent to } 1 \\
\text { year of individual } \\
\text { income }\end{array}$ \\
\hline
\end{tabular}




\section{Technology Costs}

The success of cost effective national TB programs depends on the availability of accurate and affordable diagnostic and treatment technology. Researchers and policymakers agree that current diagnostic technology is inadequate to address global demand. Given that one third of the world is believed to have undiagnosed disease, under diagnosis remains an important challenge to global TB control. The epidemiology of TB and its association with the poor in both high and low resource countries also has financial implications for diagnostic technology. Therefore, the design of effective TB screening programs along with the use of cost effective diagnostic methods remains a common discussion the peer-reviewed published literature. The appropriate use of resources has also been the primary endpoint for several studies examining current diagnostic methods.

The New Diagnostics Working Group of the Stop TB partnership published a report that summarizes the current state of TB testing technology while summarizing the challenges in new technology development. The report also included a wish list outlining the characteristics of the ideal testing methodology. ${ }^{229} \mathrm{~A}$ WHO report addressed the unmet needs of TB diagnostics offering a more detailed wish list for TB diagnostic test characteristics. The WHO also described the need for the availability of different tests relative to care setting and populations. ${ }^{230}$ 
In a review specifically evaluating diagnostic technology available to low resource countries with a higher proportion of TB, Molicotti and colleagues discuss the cost implications of diagnostic methods relative to effectively diagnosing disease. Modeling studies have shown 400,000 lives could be saved annually with the adoption of rapid TB testing techniques with a sensitivity greater than $85 \%$ for both smear-positive and smear-negative cases along with a specificity of $97 \% .{ }^{231}$ Given current technology and cost, the WHO recommends liquid culture systems in low resource settings. However, the purchase and maintenance of laboratory instruments along the laboratory infrastructure to conduct liquid culture testing simply does not exist in many low resource countries. ${ }^{232}$ Instead, many low resource countries continue to rely on the microscopic examination using the Ziehl-Neelsen smear method given its low cost of approximately $\$ 0.50$ for two sputum smear studies. However, poorly maintained laboratory equipment, understaffing, and the lack of consistently available clean water and electricity severely compromise this the sensitivity of this test. The Ziehl-Neelsen has been proven especially insensitive in populations with a compromised immune system and in pediatrics. ${ }^{233}$ Further, an important limitation of smear testing alone is that this laboratory method does not distinguish TB from other mycobacterium species or determine drug susceptibility, which contributes to inappropriate antibiotic use and the escalation of healthcare costs in these care settings. 
Although many studies acknowledge the importance of cost effective diagnostics, especially for low and middle resource countries, few studies are available that standardize and measure the of cost diagnostic technology. One cost study evaluated the affordability of new tests in 36 high burden countries. New diagnostic test types were categorized into four groups: rapid-sputum, non-sputum biomarker, triage test followed by confirmatory testing, and drug susceptibility testing. The costs of these tests were estimated based on the unit cost multiplied by the number of tests and compared with the current spending on conventional diagnostics. The conclusions of this study found that non-sputum biomarker testing and triage testing followed by confirmatory testing are affordable with current resources. The use of rapid-sputum tests and drug susceptibility testing would only be possible with additional funding. It is important to note that this study only considered variable testing cost, ignoring fixed costs such as equipment and facilities. ${ }^{234}$ In developed countries where the investment in additional infrastructure and equipment costs may not be necessary, the examination of variable cost alone might provide a complete enough picture. For low resource countries, especially where infrastructure is lacking and equipment may be unsuitable, fixed costs represent a critical part of the equation. Another challenge in estimating cost lies in the high variability of both unit and secondary material cost. For example, smear cost data vary between $\$ 0.26$ and $\$ 10.5$ and culture cost ranges from $\$ 1.63$ to $\$ 62.01$. The cost range for a 
combination unit consisting of both smear and culture ranges from $\$ 2.27$ to $\$ 48.23 .^{235}$

In order to accurately represent cost, some studies have sought to measure the economic impact of TB. Economic costs include a variety of characteristics beyond the variable costs associated with a laboratory test, but also include the investment of additional resources in order to be able to conduct the test properly. These costs may be offset by the economic impact of the test by diagnosing TB in populations earlier or detecting the disease in populations in which the disease may be undiagnosed. In these cases, economic cost would be heavily impacted by the initiation of therapy earlier, the reduction of transmission, fewer wages lost, and fewer complications of treatment. Sohn and researchers evaluated several cost studies in a review noting the difference in financial versus economic costs in TB diagnostic testing. According to this assessment, the cost of diagnostic testing extends well "beyond the simple boundaries of current consumable costs and simple comparisons of equipment cost." Instead, the need to measure economic cost as a complete assessment of cost is necessary to establish evidence-based guidelines. ${ }^{236}$

\section{Drug Resistance Cost}

The cost of TB treatment is exacerbated following a diagnosis of multi-drug resistant TB (MDR-TB) or extreme drug-resistant (XDR-TB). MDR-TB is defined by drug resistance to specific first line therapies isoniazid and rifampin. XDR-TB is likewise resistant to first line therapies 
in addition to any fluoroquinolone and one of the following three injectable second-line drugs: amikacin, kanamycin, and capreomycin. ${ }^{237,238,239}$ In 2010 , an estimated $.4 \%$ of TB cases were drug resistant worldwide, which equates to 440,000 cases annually. ${ }^{240}$ This number is expected to increase as inadequate treatment coupled with the transmission of drug resistant strains of disease are expected to continue. Official numbers, however, remain unreported. According to the WHO, less than 30,000 cases of M/XDR-TB were reported in 2008, which is believed to represent only $7 \%$ of new cases that same year. Also, WHO guidelines for the management of M/XDR-TB were unmet in $60 \%$ of reported cases. ${ }^{241}$

Treatment costs for X/MDR-TB alone range from 9 to 25 times the cost of treating drug susceptible TB, and treatment courses can be up to 3 times standard duration. ${ }^{242,243}$

The financial impact of MDR-TB disease on the patient and his or her family is not well described. A 2010 study interview-based in Ecuador documented total out of pocket treatment costs reported by patients following 2 months of therapy, noting the average for drug susceptible patients with TB at $\$ 960$ as compared to $\$ 6880$ for patients with MDR-TB. Of the 118 patients interviewed for this study, only 14 patients were infected with MDR-TB. ${ }^{244}$ In national budgets, the relatively low incidence of MDR-TB and XDR-TB commands a disproportionately high percentage of national TB program budgets. In South Africa, for example, MDR-TB accounted for $2 \%$ of TB cases in 2011 while consuming over $30 \%$ of the 
total national budget during the same year. ${ }^{245}$ The continued spread of MDR-TB and XDR-TB in low-resource countries can be potentially devastating to governments with high TB prevalence along with increasing MDR-TB and XDR-TB. In some countries, these direct medical care expenses are shared between governments and TB patients and their families, resulting in both bankrupting national governments and facilitating poverty in vulnerable populations. Also, indirect medical costs can be even more economically devastating as compared to drug susceptible TB. The consequences of more severe disease are longer treatment courses resulting in longer periods of travel, food costs, and lost wages.

A systematic review evaluating the cost of MDR-TB treatment along with identifying cost drivers following the assessment of 420 studies published in five languages found only four studies with quality data that could be extracted and adjusted to American dollars and international dollars as well as adjusted for inflation for comparison within a common year. These four studies contained cost data from care settings in Peru, the Philippines, Estonia, and Russia, noting that the largest influence on cost was hospitalization. Many national TB programs use hospital care settings to ensure patient compliance and prevent disease transmission, but the authors note that the use of outpatient care settings represent a better use of resources even with the added expense of food and travel vouchers. The use of vouchers has been proposed by the WHO to reduce 
the impact of indirect cost on patients and their families but also better ensure patient compliance to treatment courses. ${ }^{246}$ The second largest cost driver across studies was the use of expensive second line drugs, which is an unavoidable expense for patients with resistant disease. The authors suggest that despite the cost of expensive therapies, MDR-TB management delivered in an outpatient setting can still be cost effective in low and middle resource countries. ${ }^{247}$ This study also offered important implications for the availability of cost data specifically for MDR-TB when a systematic review yielded less than $1 \%$ of studies evaluated that included enough quality data to be included. Clearly, the cost of MDR-TB has not been adequately measured. A literature review searching for comparable cost data for XDR-TB revealed no reliable data from any care settings in the developing world. See the table below for a summary of costs measured in the literature associated with treating drug resistant TB. Table 9: MDR-TB in Resource Scarce Care Settings

\begin{tabular}{|c|c|c|c|c|}
\hline Author(s) & Setting & $\begin{array}{l}\text { Study } \\
\text { Type }\end{array}$ & Study Size & Findings \\
\hline $\begin{array}{l}\text { Rouzier et } \\
\text { al. }\end{array}$ & Ecuador & Interview & $\begin{array}{l}188 \\
\text { respondents }\end{array}$ & $\begin{array}{l}\text { Drug costs: } \$ 960 \\
\text { for TB, } \$ 6880 \text { for } \\
\text { MDR-TB }\end{array}$ \\
\hline $\begin{array}{l}\text { Pooran et } \\
\text { al. }\end{array}$ & $\begin{array}{l}\text { South } \\
\text { Africa }\end{array}$ & $\begin{array}{l}\text { National } \\
\text { datasets }\end{array}$ & $\begin{array}{l}\text { Population } \\
\text { based data }\end{array}$ & $\begin{array}{l}\text { MDR-TB } 2 \% \text { of } \\
\text { cases, } 30 \% \text { of } \\
\text { national budget }\end{array}$ \\
\hline $\begin{array}{l}\text { Fitzpatrick } \\
\text { and Floyd }\end{array}$ & $\begin{array}{l}\text { Peru, } \\
\text { Philippines, } \\
\text { Estonia, } \\
\text { Russia }\end{array}$ & $\begin{array}{l}\text { Systematic } \\
\text { Review }\end{array}$ & 420 studies & $\begin{array}{l}\text { Estonia: } \$ 10,880 \\
\text { Peru: } \$ 2,423 \\
\text { Philippines: } \$ 3,613 \\
\text { Russia: } \$ 14.657 \\
\text { Cost drivers: } \\
\text { hospitalization and } \\
\text { second line drugs }\end{array}$ \\
\hline
\end{tabular}




\section{TB in the developed world}

The epidemiology of TB in the developed world exists in sharp contrast to that of the developing world. In local populations, TB rates in high resource countries are associated with several distinct factors. First, the use of immune suppression therapies for cancer treatment, posttransplant therapy or immune modulation therapy for auto-immune diseases have created a risk group that only exists in resource rich countries where advanced therapy options are widely available. Also, comorbid conditions that affect the immune system including underlying pulmonary diseases, diabetes mellitus, ${ }^{248}$ and advanced HIV/AIDS is a risk factor for active TB all over the world including the industrialized west. ${ }^{249}$ Behavioral factors play an important role such as diet and malnutrition, alcoholism, and smoking tobacco, particularly in vulnerable populations. ${ }^{250}$

In the industrialized west, social determinants of health are strong predictors of TB infection. In a master's thesis, the disparity of disease incidence in local populations was studied in both Canada and New Zealand. In both countries, a higher incidence of TB has been recorded in local aboriginal populations in Alberta, Canada as well as the Maoria and Pacific populations in New Zealand. This study identified key individuals involved in the transmission of TB within their communities based on contact tracing and strain analysis, noting that substance use and a delay 
in accessing treatment were common characteristics from both populations. The author notes that future research in this area might solidify this association by studying the impact of other characteristics of vulnerable populations such as poverty and unemployment. ${ }^{251}$ Despite the presence of these risk factors in key populations in North America and Europe, TB rates have remained stable or declined in local populations.

Immigration remains one of the strongest predictors of TB infection in North America and Europe, particularly those who migrated from high HIV and TB burden countries. In fact, more than half of active TB cases are diagnosed in immigrant populations. ${ }^{252,253}$ In the UK, the incidence of disease in local populations has fallen $5 \%$ between the years 1998 and 2008 and likewise increased by $94 \%$ in foreign-born populations during the same years. ${ }^{254}$ In the years $2000-2013$, TB cases in locally born populations have actually decreased by half in the US, Canada, and Europe while rates among immigrant populations have increased. The European Centre for Disease Prevention and Control and the WHO Regional Office for Europe released a joint report in 2015 noting that $28 \%$ of TB cases in Europe in 2013 were in foreign-born populations. ${ }^{255}$ In Western Europe, a 2010 report estimates this average is excess of $40 \% .{ }^{256}$ In some states, the prevalence of disease in the foreign-born account for a resounding majority: Luxemburg (94.7\%), Sweden (88.7\%), Malta (88.0\%), Norway (86.0\%), Cyprus (85.4\%), Netherlands (73.9\%), Switzerland (75.3\%), and United Kingdom (70.1\%). ${ }^{257}$ In the United 
States, the CDC reported that $64.6 \%$ of TB patients were born outside the US in $2013 .{ }^{258}$ Immigration rates to the US are expected to continue if not increase in coming years. Each year, approximately 450,000 persons are admitted to the United States on an immigrant visa, and 50,000-70,000 are admitted as refugees. ${ }^{259}$

\section{Migrant Populations}

Immigration status alone is not a predictor for TB because the prevalence of disease is much more pronounced in select immigrant groups than others. Among the immigrant populations at a particularly high risk are those from moderate to high TB burden countries. Also, immigrants having arrived within 5 years, termed "new entrants," are another important risk group. ${ }^{260}$ In the UK, new entrants are believed to account for an estimated $50 \%$ of TB cases in the foreign-born population. ${ }^{261}$ Finally, immigrant populations with comorbidities represent important risk groups for developing active disease such as HIV/AIDS. ${ }^{262}$ According to the European Centre for Disease Prevention and Control and the WHO Regional Office for Europe, data were available in 36 countries regarding TB and HIV noting a co-infection rate of $7.8 \%$. The 2012 joint analysis reported a co-infection rate of $6.1 \%$, which confirms the $18 \%$ increase in co-infection rates annually that has occurred in Europe since 2004. It is important to note that this figure only includes data from 36 reporting countries, and only 219,095 of 324,084 TB patients had a documented history of HIV testing. Therefore, it is likely that this 
documented co-infection rate is under-reported. ${ }^{263}$

The presence of TB in immigrant populations can be explained by three important pathways. First, immigrants arriving to destination countries may have been previously exposed and infected with active disease while residing in their home countries and represent cases of active disease upon arrival. Also, immigrants may be infected with latent disease upon arrival, which activates following entry into destination country. Third, immigrant populations acquire TB from local transmission following exposure most likely to peers from moderate to high burden countries. ${ }^{264}$ Immigration from these countries is expected to remain a key factor in TB epidemiology as the United Nations note immigration figures in 2013 alone was 232 million, representing a 50\% increase over the preceding two decades. The $2013 \mathrm{UN}$ report explains migration is expected to continue to increase secondary to globalization, conflict and financial and economic reasons. ${ }^{265}$

The impact of immigration on TB rates is expected to be more measurable given the recent refugee crisis secondary to current conflicts in Iraq and Syria. According to Amnesty International, over half of Syria's population is currently displaced. While some remain displaced within Syria, the majority of refugees reside in neighboring countries including Turkey, Lebanon, Jordan, Iraq and Egypt. ${ }^{266}$ In 2015, European countries noted a major influx of Syrian refugees into Europe, coming from both Syria and refugee centers in neighboring countries. The number of first 
time applicants for asylum in European Union countries more than doubled from 563,000 in 2014 to almost 1.26 million in 2015. Syrian refugees represented $29 \%$ of the total number of first time applications in $2015,14 \%$ from Afghanistan, and 10\% from Iraq. ${ }^{267}$ The US has pledged to accept 10,000 Syrian refugees in 2015 , increasing allowances in the three subsequent years for an average of 70,000 to 85,000 annually. ${ }^{268}$ As of February 2016, Canada has accepted over 25,000 Syrian refugees. ${ }^{269}$ These numbers exist in excess of regular and projected total immigration numbers including asylum seekers, and represent important implications for immigration, population demographics and resulting public health policy in many countries. Regular immigration in the US includes approximately 450,000 persons using immigrant visas and between 50,000 and 70,000 as refugees. ${ }^{270}$

Many industrialized states have created and executed extremely successful public health programs that have controlled TB rates as evident in its decline in local populations. These programs seek to include the diagnosis of TB in early stages of disease, initiate treatment while ensuring patient compliance, and trace possible transmission contacts. However, this approach is only relevant in cases in which TB is acquired following arrival to destination countries and fails to identify and treat cases of latent and active disease present in immigrant populations during the migration process. Policies specifically designed to target migrant populations represents an emerging need in many countries as part of a 
more comprehensive TB program aimed at reducing the burden of disease both local and migrant populations.

In the US, programs for screening and managing related treatment for immigrants diagnosed with TB is managed according to the Directly Observed Therapy, Short-course (DOTS) national framework. ${ }^{271}$ DOTS is an approach to TB surveillance and treatment recommended by the WHO that requires first an investment from all levels of government internally as well as international partnership in order to enhance TB monitoring, surveillance and training. First, DOTS emphasizes the need for smear microscopy followed by culture and drug susceptibility testing for the accurate diagnosis of disease as well as the identification of drug resistant cases. Next, DOTS calls for a standardized treatment regimen of six to nine months in total duration, the first two months of which should be directly observed by a healthcare worker or community health worker. DOTS also calls for a standardized surveillance and monitoring systems. The overall goal of DOTS is to ensure that TB is cured in diagnosed cases following an appropriate treatment course, which will also prevent further transmission as well as the facilitation of drug-resistance. ${ }^{272,273}$

Many aspects of TB screening of immigrant populations is controversial. For starters, the use of DOTS as a comprehensive testing and treatment program has significant costs for countries with a large inflow of immigrants when the supervised treatment portion of the program has not been well proven. These findings were consistent with a 
previously published multicenter study of nearly 500 patients in which three arms were evaluated: 70 were assigned DOTS with direct observation of treatment by health workers, 165 were assigned DOTS with direct observation of treatment by family members, and 162 were assigned self-administered treatment. Outcome measures were cure or treatment completion. This study found similar outcomes in all three arms with cure or treatment completion rates of $67 \%, 62 \%$, and $65 \%$, respectively. This was an important study that failed to demonstrate DOTS as a superior strategy over other treatment programs. ${ }^{274}$ In a recent retrospective comparative study of 150 patients, the treatment success rate of $72 \%$ was equal in a sample of 75 who underwent compulsory supervised treatment versus the remaining 75 whose care was delivered in a self-administered model. Given that DOTS remains unproven as a superior approach to the administration of TB therapy, some policymakers recommend its use only after patients fail selfmanagement. ${ }^{275}$

Many industrialized countries have designed TB screening programs that are integrated with immigration procedures as a result of this emerging need. One of the most common screening methods used is chest radiography for the detection of active disease. ${ }^{276}$ Applicants seeking entrance into the US as an immigrant or refugee status are required to undergo a medical examination prior to their arrival. The Department of State appoints the physicians who perform the 
examinations according the Technical Instructions provided by the CDC's Division of Global Migration and Quarantine (DGMQ). Beginning in 1991, chest radiography has been used for applicants over the age of 15 years. Chest x-ray findings suggestive of pulmonary TB were further evaluated using sputum smears until 2007, when the CDC followed the sputum smear with cultures. In $2012,60 \%$ of the TB cases diagnosed using cultures were in applicants with negative smears.

Meanwhile, the universal use of chest x-ray for immigrant screening has been widely debated in the literature. Most evidence does not support its use due to unacceptable sensitivity, specificity and costeffectiveness. ${ }^{277}$ One systematic review and meta-analysis in which 22 relevant publications were analyzed agreed that chest radiograph did not offer the acceptable yield of an effective screening tool, noting that of the $2,620,739$ imaging studies only 5,446 cases of active disease were diagnosed, representing one diagnosis in 481 chest radiographs. ${ }^{278}$ Another report noted that among the important diagnostic challenges is that chest radiography depends on access to complementary resources such as equipment and the availability of experienced medical personnel who possess the skills to identify active disease with an atypical appearance. ${ }^{279}$ Further, the incidence of false positives secondary to technical problems, lung scarring, the presence of other underlying pulmonary disease, or previous TB with associated healed cavitary lesions is another important driver of program cost. These factors commonly 
result in further medical evaluation and additional costs. ${ }^{280}$ For these reasons, untargeted chest radiography has been disproven as part of a cost effective screening program.

The well documented challenges of diagnosing active TB has resulted in a lively discussion in the peer-reviewed medical literature and among policy makers theorizing more effective TB treatment and diagnostic programs in immigrant populations. This discussion has, in some cases, resulted in policy changes in some countries. Following a review of several countries in North America and Europe, it is evident that a lack of uniformity in screening methods and goals exists in developed countries. This review implies that no proven standard of TB control exists that effectively balances TB risk management with cost in immigrant populations. The most common screening practice is the single-level, one time screening of migrants for active disease. These largely superficial and ineffective screening programs remain the most common despite low yield, which is perhaps the most important measure of program effectiveness. ${ }^{281}$

The literature offers a variety of other suggestions for more cost effective screening programs for immigrant populations. One important suggestion is to target chest radiography to migrant populations with risk factors for disease. In this case, imaging may only be recommended for migrants from moderate to high resource countries or with personal risk factors for disease. The screening process in Switzerland exists as a 
model for reserving chest radiography for select populations based on results from a pre-screening interview and associated questionnaire. The Swiss program utilizes a nurse to conduct the interview in which medical history and family history are recorded and the results are measured against the risk associated with country of origin. This program reduces the number of migrants undergoing chest radiograph based on risk stratification. $^{282}$

A 2013 study that evaluated a variety of screening approaches demonstrated that screening for active disease in immigrant populations is one piece of what should be multi-faceted program to detect both active and latent disease. Further, the WHO recommended in a 2014 report that migrants from high burden TB countries to be screened for latent disease as well. ${ }^{283}$ Further, findings from a 2016 review suggest that effective screening can only be accomplished through multiple, inter-linking elements including pre-arrival screening of active disease in addition to post-arrival screening of latent disease targeted to migrants from moderate to high burden TB countries. The authors acknowledge considerable challenges with the implementation of a multi-level program, but urge more research in TB screening program design and effectiveness in order to better establish international evidence based guidelines. ${ }^{284}$

Given that the presence of latent disease is another important pathway to infection for immigrant populations, some policy makers have also suggested immigrant screening for latent disease. However, few 
studies have been published that analyze the effectiveness of screening programs for latent disease.

TB skin testing (TST) has been the screening tool of choice for a variety of settings including but not limited to healthcare and restaurant workers in addition to immigrant populations. The majority of the limited data evaluating the potential utility of latent disease screening programs in immigrant populations relies on the TST. A study of refugee populations evaluated the impact of screening for latent disease using a TST prior to entrance into the United States as a means of reducing the incidence of active TB in this population. This was a cost benefit study using a decision tree with multiple Markov nodes to determine the total costs and number of active TB cases in 55,35 , and $20 \%$ TST positive under two models: no overseas screening and overseas screening and treatment. Also, refugees were stratified by risk based on the prevalence of TB in their country of origin into the following categories: high, moderate, and low TB. Using the hypothetical 100,000 refugees over a one year period from countries with high, moderate, and low TB prevalence, the authors estimated that screening for latent TB would prevent 440, 220, and 57 cases in the US during the first 20 years after arrival. Further, the authors emphasized a cost savings associated with the screening program of $\$ 4.9$ million for refugees from high burden TB countries when the cost of treatment was considered, $\$ 1.6$ million for countries with moderate TB prevalence, and $\$ 780,000$ for low TB prevalence countries. The authors 
noted that these cost savings were conservative given that the cost of managing additional cases secondary to the transmission of active TB from these cases was not considered in this model. ${ }^{286}$

One important consideration of screening for latent TB is the several functional challenges surrounding the use of the TST. First, the test is read between 48 and 72 hours following administration, which requires a separate visit. Also, the reading of results may be open to interpretation. ${ }^{287}$ The other potentially critical challenge is relying on the TST especially when screening immigrants from high burden TB countries is previous exposure to the Bacillus Calmette-Guérin (BCG) vaccine. In many high burden TB countries, the BCG vaccine is administered to babies as a population level prevention strategy. ${ }^{288}$ In fact, the WHO recommends that BCG be administered to all children born in high burden countries. ${ }^{289}$ In low and moderate burden countries, the use of BCG vaccine is targeted to at risk populations. Babies in these populations are sometimes vaccinated. Adults can be vaccinated as well. The vaccine protection timeframe is between 10 and 20 years. ${ }^{290}$ One major consideration, however, is research indicates that exposure to the BCG vaccine can generate a false-positive TST. Also, those with active NTM infection are likely to yield a false positive result. ${ }^{291,292}$ Further, TST may generate false negatives in hosts who are pregnant, or immunosuppresses due to malignancy, nutrition, HIVIAIDS, or those receiving immune modulating therapy given the reliance of the TST on the 
ability of the immune system to generate a response. ${ }^{293,294,295}$

Another important screening tool for latent disease are IFN-gamma release assays (IGRAs). Currently, two blood assay tests are commercially available: Quanti-FERON-TB Gold In-Tube (QFT-GIT; Cellestis, Carnegie, Australia) whole blood enzyme-linked immunosorbent assay (ELISA), and the T-SPOT.TB assay. Limited data are available that compares TST with assays in migrant populations, noting that QuantiFERON-TB seems to have a lower detection rate than the T-SPOT.TB assay. It is important to note, however, that both assays seem to be less sensitive than TST. ${ }^{296}$ Also, few data are available to evaluate the use of IGRAs as a screening tool, but emerging data indicates that blood assays are not reliable in this setting, especially in cases in which latent disease has been previously treated or there has been a history of TST. ${ }^{297}$

In a master's thesis, the cost effectiveness of screening tools including TST and blood assay tests for latent infection were evaluated in the Netherlands, a low burden TB country, in immigrant populations who participate in contact investigations. Using decision tree analyses that measured direct medical costs for two years following screening for latent disease, this study measured two years of cost analyses following initial contact investigation in which the use of TST was validated in healthy immigrant contacts. ${ }^{298}$

Perhaps the most important component of a successful screening 
program for latent disease is the ability to provide and complete treatment in positive cases. This depends on two primary factors. First, adequate public resources need to be allocated to provide treatment in all cases of latent infection. Second, a concentrated effort is necessary in order to assure that immigrant populations remain compliant during long treatment courses. This remains a particular challenge given that these populations tend to be vulnerable and not always able to comply with office visits and medication. Also, in many migrant populations people move frequently, which requires a transfer of care during long treatment courses, which increases the likelihood of patients discontinuing therapy. Further, cultural issues in many populations play an important role. In a study of Hispanic migrant farm workers entering the US, adherence to treatment was found to be an important challenge noting that less than half agreed to even initiate therapy citing cultural barriers. ${ }^{299}$

These programs all share an important limitation in that they are confined to a screening population of migrants that legally enter destination countries and submit to the legal entry process. Population screening is not available for the thousands of migrants who enter destination countries illegally. ${ }^{300}$ The Department of Homeland Security estimates 11.4 million unauthorized immigrants were living in the United States on January 1, 2012. This figure has not significantly changed from the January 1, 2011 estimate of 11.5 million. ${ }^{301}$ 


\section{Nontuberculous Mycobacterium}

Nontuberculous Mycobacterium (NTM) represents an emerging public health challenge. In the developed world, NTM infection rates have risen significantly in recent decades secondary to many factors including population aging, the availability of immune suppressing therapies in resource rich healthcare delivery systems secondary to cancer, autoimmune disorders and post-transplant therapy. Also the worldwide HIVIAIDS epidemic continues to facilitate the prevalence of NTM in the developed world as well as the undeveloped world. Even so, cost information measuring direct and/or indirect costs as well as economic impact remains extremely under measured. The few available studies that attempt to describe the cost of care of NTM in limited care settings note the lack of data available and encourage other research in this largely unappreciated global health crisis. In the undeveloped world, even less data are available regarding all aspects of NTM including epidemiology and cost. The majority of discussion on NTM in the developing world addresses the disease as it impacts the diagnosis and treatment of TB. These studies emphasize the challenge of appropriate treatment for TB in high burden clinical settings when the potential confounding case of NTM presents.

\section{NTM in the underdeveloped world}

The epidemiology and cost of NTM in the developing world is an emerging area of research in the field of mycobacteriology. Many 
important challenges exist that has made research in this area difficult. For starters, mycobacterial disease is diagnosed in developing countries based on clinical and radiological findings. ${ }^{302}$ Diagnostic technology is not usually available in these care settings that would differentiate NTM species from TB or other diseases. Given that NTM often resembles TB in disease presentation and radiological features, many researchers believe that NTM is often mistaken for TB, particularly MDR-TB, following a treatment failure. This theory has important implications for resource management in low resource care settings as well as antibiotic stewardship. For these reasons, no estimates of NTM rates in low resource countries are currently available. What little data exists must be pieced together from studies conducted of TB in low resource countries in which the discovery of NTM is incidentally reported. ${ }^{303}$ No cost information is available except also in cases in which NTM was misidentified and noted to impact the cost of treatment.

Emerging literature is addressing the burden of NTM disease in low resource countries as its clinical importance relating to other health challenges in these care settings has become increasingly apparent. First, the association of NTM with the HIVIAIDS epidemic is well described in most every care setting. The importance of this relationship is amplified in developing countries in sub-Saharan Africa in which HIVIAIDS rates have been reported as high as $25 \%$. Further, the unavailability of important therapies that have been shown to slow down or stop the 
progression of HIV to AIDS in many low resource care settings is also an important contributor to the epidemiology of NTM. In a 2007 dissertation in which NTM was studied in Zambia, one of the world's poorest countries, this particular study emphasized the importance of HIVIAIDS as a risk factor for NTM in Zambia as well as neighboring countries in the region. This dissertation featured a study population of 64 patients of mixed HIV status who had been chronically ill for more than two weeks, thirty of which were identified as infected with mycobacterium disease using liquid culture. Only eight patients from this sample were found to be infected with TB as compared to twelve who were infected with NTM. Of the NTM species identified, seven patients were diagnosed with $M$. szulgai, three patients had MAC, one patient had $M$. simiae and the remaining one patient had $M$. terrae. One interesting finding of this study was the presence of $M$. szulgai, which represents an NTM species that strongly resembles TB in disease presentation as well as DNA. In this particular sample, five patients with NTM disease caused by M. szulgai were given anti-TB therapies and three cases were noted as responsive. It is also important to note that ten isolates in this study were unable to be identified. The major conclusion of this study was that TB is grossly over diagnosed in Zambia, which must be addressed by improving access to better diagnostic technology as well as the incorporation of diagnostic scoring systems and algorithms to be validated for use in low resource countries with a high burden of TB and HIVIAIDS as diagnostic support 
tools. $^{304}$

In a study conducted in the high burden TB country Mali where most patients receive empiric treatment for TB, clinical samples from 142 patients were evaluated by smear, culture and drug susceptibility testing. This study had two arms: 61 patients who had undergone treatment for TB and 17 patients who had no previous exposure to anti-TB drugs. Of the 11 patients who tested positive for NTM, all were identified from the treatment experienced group. It is important to note that all 11 candidates had been identified as candidates for expensive second line anti-TB treatments. ${ }^{305}$

In low resource countries with reliable access to laboratory diagnostics, the low cost option of sputum smears remains widely used. The issue with smears, however, is that TB and NTM cannot be differentiated without follow up studies including cultures or molecular methods of species identification. ${ }^{306}$ In China, $80 \%$ of laboratories designated for TB diagnostics lack the equipment necessary to perform culture or other methods of species identification leading to a diagnosis of NTM infection. ${ }^{307}$ The misdiagnosis of NTM infection has important global consequences, leading to inappropriate treatment, elevated cost, and the facilitation of continued antibiotic resistance.

In a study conducted in Tehran, Iran, sputum smear and culture were performed on clinical isolates collected from 105 patients. 
Microbiological testing was repeated twice to assure accuracy. Also, PCR was performed on all patients and species identification was performed by PCR and routine identification tests for all culture-positive cases. In this study, 12 isolates, $(11.43 \%)$ were identified NTM species. It is important to consider that clinical appearance was similar in patients. For example, cavitary lesions were present in $41.7 \%$ of the cases. ${ }^{308}$

Another study conducted in the southwest portion of Iran was a retrospective evaluation of 117 consecutive samples collected from patients diagnosed with MDR-TB. Patients who met criteria for inclusion had been administered between two and three months of a first line antiTB regimen but had not achieved sputum conversion. A total of 35 patients in this sample had isolates that were identified as an NTM species, and 32 of these patients met American Thoracic Society (ATS) and the Infectious Diseases Society of America (IDSA) criteria for active NTM disease. Underlying disorders that represent risk factors for NTM were present in 32 patients and included active malignancy, organ transplantation, HIV, chronic renal failure, and diabetes mellitus. Only three patients diagnosed with NTM in this study had no known risk factor. This study also evaluated clinical outcomes for 27 patients retrospectively identified with NTM for which this data was available. Ten patients were noted to have poor outcomes, six of which were documented as treatment failures and four died during the treatment course. The remaining 17 patients for which outcome data was available was noted as cured, 
although the authors admit that outcome data was difficult to ascertain and is likely inaccurate. The importance of this study is that it demonstrated that NTM was identified in $30 \%$ cases that had been misdiagnosed with MDR-TB, which represents a figure that is higher than the previous reports from Iran. It is also important to note that given this study being limited only to MDR-TB cases, the case of mistaken identity in mycobacterial diseases is likely a much bigger problem than current literature has proven. The authors of this study ask an important question, is MDR-TB really NTM? ${ }^{309}$

A study conducted in Nigeria found that of the 1603 patients with assumed TB, only 444 yielded a positive culture result following laboratory microbiological studies. Of these, 69 isolates were found to be infected with NTM. This study also found that NTM was more frequently diagnosed during the season of the year characterized by the presence of dust, validating environmental exposure as an important transmission pathway. Also, the presence of NTM infection in younger hosts was frequently associated with the presence of comorbid HIVIAIDS as compared to those older than 35 years. ${ }^{310}$

The burden of NTM in low resource countries has been studied mostly in the context of identifying functional barriers in the appropriate management of TB. Due to the similarities in symptomology coupled with the difficulty of diagnostics when relying merely on smears, the two are easily confused in low resource settings, leading to improper treatment, 
increasing costs and facilitating further drug resistance. See the table below for a summary of NTM studies from the underdeveloped world.

Table 10: NTM studies in the underdeveloped world

\begin{tabular}{|l|l|l|l|l|}
\hline Author(s) & Setting & Study Type & Study Size & Findings \\
\hline $\begin{array}{l}\text { Buijtels, P } \\
\text { (doctoral } \\
\text { dissertation) }\end{array}$ & Zambia & $\begin{array}{l}\text { Laboratory } \\
\text { based using } \\
\text { liquid culture }\end{array}$ & $\begin{array}{l}\text { 64 patients } \\
\text { with mixed } \\
\text { HIV status }\end{array}$ & $\begin{array}{l}\text { 30 Mycobacterium; } \\
\text { 8 TB; 12 NTM }\end{array}$ \\
\hline $\begin{array}{l}\text { Maiga et } \\
\text { al. }\end{array}$ & Mali & $\begin{array}{l}\text { Laboratory } \\
\text { based: } \\
\text { smear, } \\
\text { culture, DST }\end{array}$ & $\begin{array}{l}\text { 142 patients: } \\
\text { 61 treatment } \\
\text { experienced; } \\
17 \text { treatment } \\
\text { naive }\end{array}$ & $\begin{array}{l}\text { 11 patients } \\
\text { diagnosed with } \\
\text { NTM, all from } \\
\text { treatment } \\
\text { experienced group }\end{array}$ \\
\hline $\begin{array}{l}\text { Tabarsi et } \\
\text { al. }\end{array}$ & $\begin{array}{l}\text { Tehran, } \\
\text { Iran }\end{array}$ & $\begin{array}{l}\text { Laboratory } \\
\text { based using } \\
\text { culture and } \\
\text { PCR }\end{array}$ & $\begin{array}{l}105 \text { patients } \\
\text { diagnosed with } \\
\text { NTM }\end{array}$ \\
\hline $\begin{array}{l}\text { Shahraki } \\
\text { et al. }\end{array}$ & $\begin{array}{l}\text { South- } \\
\text { west } \\
\text { Iran }\end{array}$ & $\begin{array}{l}\text { Laboratory } \\
\text { studies } \\
\text { conducted } \\
\text { on patients } \\
\text { diagnosed } \\
\text { and treated } \\
\text { for MDR-TB }\end{array}$ & $\begin{array}{l}117 \text { patients } \\
\text { who had not } \\
\text { improved } \\
\text { after 2-3 } \\
\text { months of } \\
\text { anti-TB } \\
\text { therapy, }\end{array}$ & $\begin{array}{l}\text { 35 patients with } \\
\text { cultures positive } \\
\text { for NTM, 32 met } \\
\text { ATS/IDSA criteria } \\
\text { for infection }\end{array}$ \\
\hline Aliyu et al. & Nigeria & $\begin{array}{l}\text { Laboratory } \\
\text { based using } \\
\text { culture }\end{array}$ & $\begin{array}{l}\text { 1603 } \\
\text { patients } \\
\text { diagnosed } \\
\text { clinically with } \\
\text { TB }\end{array}$ & $\begin{array}{l}\text { TB: 444 patients; } \\
\text { NTM: 69 patients }\end{array}$ \\
\hline
\end{tabular}

\section{NTM in the developed world}

The relationship between TB and NTM in the developed world has been dynamic in recent years. While TB rates in North America, Europe and parts of Asia have been steadily declining, the prevalence of NTM disease in the same care settings has exploded. To illustrate, NTM infection prevalence in North America was estimated to be between 1.6 
and 1.8 per 100,000 in the 1980 s, with reports published in 2007 estimating prevalence of 14.1 per $100,000 .^{311}$ It is likely that this number is even greater. A 2014 study sought to establish the relationship between NTM and TB in different care settings all over the world. A total of 16 regions were evaluated including areas in Canada, the US, western Europe, Australia, Greece, Czech Republic and Japan, and the decline of TB alongside the incline to NTM was noted in the majority of regions based on data published in 22 studies. The authors offered several theories for this relationship. First, the existence of some degree of crossimmunity between different mycobacterial species could be a consideration if both diseases were affecting similar populations. Given that TB and NTM affect characteristic populations that do not intermingle; this explanation does not explain the trend particularly in North America and Europe. Another theory is related to stages in modernization. In North America and Europe, some of the causal elements that created a surge in TB rates in the nineteenth century urban crowding and issues with sanitation secondary to industrialization. In many of these modern cities, significant progress has been made to improve urban crowding and sanitation with improvements in ventilation and public water delivery systems. Given that NTM species have been confirmed in a variety of water samples including municipal water pipes and can be theoretically transmitted through aerosolized water from showers and hot tubs, it is conceivable that modern advances particularly in water delivery systems 
have facilitated the transmission of NTM. Of course, improvements in laboratory methods that may have misdiagnosed NTM as TB may also play a critical role. Also, population aging and the age of immunecompromising conditions and drug therapies also play an important role. ${ }^{312}$ Finally, the worldwide HIVIAIDS epidemic has had an impact on the prevalence of disease in the developed world as well as North America and Europe. ${ }^{313}$

\section{NTM and Cost}

A total of four studies have been published that attempt to measure the cost of NTM infection in western care settings. In the United States, a small cohort study of 27 patients with pulmonary NTM found an annual antibiotic therapy cost of $\$ 5,196$ for those infected with MAC and $\$ 11,796$ for M. abscessus. The median total per patient drug cost was reported at $\$ 19,876$ Also emphasized in this study, the cost of treating different types of NTM species can fluctuate due to variations of antibiotic cost. For instance, $M$. abscessus was noted to be associated with higher treatment cost as well as the use of intravenous antibiotic therapy over oral. Ballarino noted that given cost data is limited in the literature for NTM, the use of a more established disease such as HIVIAIDS given its chronicity and high medication cost might be an effective proxy for NTM. ${ }^{314}$

A retrospective chart review was conducted in Ontario, Canada of pulmonary NTM to determine outpatient treatment costs in Canada. ${ }^{315}$ Of the 177 patients identified in this study, costs were calculated for the 91 
who underwent treatment for an average duration of 14 months.

Researchers found that annual cost was $\$ 5,000$ in Canadian currency adjusted to 2008 value and antibiotics were responsible for $70 \%$ of total cost. It is also important to consider that over $40 \%$ of patients had been diagnosed with pre-existing COPD, and an even higher proportion of patients had been diagnosed with obstructive airway disease. Also noting the unavailability of cost data for NTM, Leber and Marras suggest using diabetes mellitus as a proxy for measuring cost. ${ }^{316}$

Perhaps the most comprehensive studies currently available are the two that used national data to estimate and report national epidemiological and cost data. The first is a state specific population based study using Medicare data along with national survey data. Strollo and researchers used a $5 \%$ sample size of the Medicare Part B database during the years 2003 through 2007 in order to establish an annual prevalence, and applied these averages to census data from 2010. According to this study, an estimated $\$ 815$ million was spent on NTM care in $2010,87 \%$ of which was spent on hospitalizations and $13 \%$ on outpatient management. This study also estimated that $76 \%$ of direct medical costs was used for antibiotic therapy. Given that this data would only include Americans over the age of 65 years, this study relied on previously reported epidemiology in peer-reviewed literature in order to estimate the number of cases in this age group. Cost was also estimated based on figures reported in other studies and adjusted to the 2014 value 
of the U.S. dollar. Further, cost figures reported in this study only included one medical encounter per year per patient. For inpatient estimates, cost would only include one hospitalization, which may be incomplete given that NTM infection has high rates of recurrence and other complications that often result in readmissions. For outpatient data, this study only included one NTM-related visit per patient per year, which is more likely to be incomplete given the chronic nature of NTM infection and its requirement of routine outpatient visits. Also, the need for close monitoring of patients undergoing treatment for NTM is necessary in order to both measure treatment success and identify adverse reactions quickly that are common during an NTM treatment course. Although this study boasts of being the very first to provide state level NTM epidemiological and cost data, the figures reported represent merely estimates that are likely significantly lower than actual figures. Despite this, the value of this study is its demonstration of NTM as a substantial disease burden with high financial costs. ${ }^{317}$ Another national study measured epidemiological data and cost associated with the management of ten waterborne diseases. The most common pathogens in this study associated with disease were NTM along with giardiasis, cryptosporidiosis, Legionnaires' disease, and otitis externa. This study used national data available from the CDC that included claims data from Medicare and Medicaid for the most recent two-year period available during the study period. Medicare data available through the CDC was accessed through Marketscan ${ }^{\circledR}$ and 
was available for the years 2006 and 2007. Multi-State Medicaid databases were available through the CDC from Thomson Reuters from the years 2004 and 2005. From 3,684 hospitalizations and 341,157 outpatient visits, NTM was found to be among the most important of waterborne disease representing the cause of disease in $21 \%$ of immunocompromised patients, second only to cryptosporidiosis at $24 \%$. Patients with NTM had the longest reported hospital length of stay at an average of 10.5 days. Also, pulmonary NTM was the most expensive disease to manage in an outpatient setting with collections ranging from $\$ 311$ from Medicaid patients to $\$ 1848$ for patients with commercial insurers. These values represents the amount collected from payers per visit. Also, NTM represented the second highest average hospitalization cost with pulmonary infection collections ranging from $\$ 12,725$ for Medicaid to $\$ 37,579$ for commercial insurance per episode. For pulmonary NTM, this study recorded a total of 22,210 cases, 14,210 $(65 \%)$ were managed in an outpatient setting with the remaining 7,659 (35\%) represent hospitalized patients. The average hospitalization collection reported in this study was $\$ 25409$, and the average outpatient cost was $\$ 1374$. It is important to note that this figure did not include laboratory testing costs. The purpose of this study was to highlight the significance of waterborne disease given the near-universal exposure to multiple water sources that can lead to significant costs in the event of a community-wide outbreak. However, this study had several important 
limitations. First, many of the diseases evaluated have several routes of transmission so the impact of waterborne diseases remains unproven. As is the case with most studies, measuring the total economic burden of diseases that includes mortality and disability, work and time loss, and chronic sequelae are difficult to measure, so this study that seeks to establish the costs of direct medical care only will always be incomplete. In this particular study, some aspects of direct medical costs had to be estimated as some outpatient data was unavailable. Also, this study was limited to patients with Medicare and Medicaid as payers for outpatient data, which has important patient selection implications for age and demographics. $^{318}$

Currently available data is limited to four studies that measure some aspects of direct cost for pulmonary NTM using data obtained from different care settings and patient populations. Also, these costs are represented in different currencies and inflationary values. As a result, cost findings for all four studies have been represented as an average per patient cost value and adjusted to US currency using Google currency converter $^{319}$ and 2016 value using the Consumer Pricing Index calculator available through the US Bureau of Labor and Statistics. ${ }^{320}$ These findings are outlined in the table below. 
Table 11: NTM and Cost in the Peer Reviewed Literature

\begin{tabular}{|c|c|c|c|c|}
\hline $\begin{array}{l}\text { Author(s), } \\
\text { Year of } \\
\text { Publication }\end{array}$ & Setting & Study Size & Cost Findings & $\begin{array}{l}\text { Average Cost } \\
\text { per Patient }\end{array}$ \\
\hline $\begin{array}{l}\text { Ballarino et } \\
\text { al. } 2009\end{array}$ & US & 27 patients & $\begin{array}{l}\$ 5,196 \text { for MAC } \\
\text { and } \$ 11,796 \text { for } \\
M . \text { abscessus } \\
\text { Antibiotics alone }\end{array}$ & $\begin{array}{l}\$ 19,876 \\
\text { (USD, 2008) } \\
\$ 22,315.55 \\
\text { (USD, 2016) }\end{array}$ \\
\hline $\begin{array}{l}\text { Leber and } \\
\text { Marras } \\
2011\end{array}$ & Canada & 177 patients & $\begin{array}{l}\$ 4,916 \text { for } \\
\text { outpatient } \\
\text { treatment, } 70 \% \\
\text { of total cost was } \\
\text { antibiotics }\end{array}$ & $\begin{array}{l}\text { \$4916 (CAD, } \\
2008) \\
\$ 4109.44 \\
\text { (USD, 2016) }\end{array}$ \\
\hline $\begin{array}{l}\text { Strollo et } \\
\text { al. } 2015\end{array}$ & US & $\begin{array}{l}\text { National } \\
\text { Medicare data, } \\
2010 \\
86,244 \text { patients }\end{array}$ & $\begin{array}{l}\$ 815 \text { million total } \\
\text { in } 2010,87 \% \\
\text { hospitalizations } \\
\text { and } 13 \% \\
\text { outpatient, } \\
\text { antibiotics } \\
\text { accounting for } \\
76 \% \text { of total cost }\end{array}$ & $\begin{array}{l}\$ 9453.22 \\
(U S D, 2014) \\
\$ 9,652.60 \\
(U S D, 2016)\end{array}$ \\
\hline $\begin{array}{l}\text { Collier et } \\
\text { al. } \\
2012\end{array}$ & US & $\begin{array}{l}\text { National } \\
\text { inpatient } \\
\text { sample, } \\
\text { Medicare, } \\
\text { Medicaid } \\
7,659 \\
\text { hospitalizations, } \\
14,551 \\
\text { outpatient }\end{array}$ & $\begin{array}{l}\text { Average costs: } \\
\$ 1374 \text { outpatient } \\
\text { ( } 65 \% \text { of cases) } \\
\text { and } \$ 25,409 \text { for } \\
\text { hospitalizations } \\
\text { ( } 35 \% \text { of cases) } \\
\text { for pulmonary } \\
\text { NTM }\end{array}$ & $\begin{array}{l}\$ 9.786 .75 \\
\text { (USD, 2007) } \\
\$ 11,409.27 \\
\text { (USD, 2016) }\end{array}$ \\
\hline
\end{tabular}

Other presentations of disease such as dissemination or bone and joint infection site can actually be more costly to treat. The Collier at al study does offer some limited data on general NTM infection, but does not define the site of infection. Also reported in this study is limited cost data for disseminated NTM infection. Unlike the body of literature measuring various aspects of TB and cost, similar data is unavailable that measures indirect costs and economic impact of NTM infection. 


\section{Literature Gap}

NTM is an emerging public health problem in the US and the world for which very little has been published in the current body of literature. Also, given that NTM is not a reportable disease in the US, rates of infection are estimated and pieced together from various sources as no central epidemiological or surveillance data exists. In other developed countries, the burden of the rising rates of NTM infection is likewise not understood. In the developing world, NTM is largely ignored and likely managed as TB, and is in many cases retreated as MDR-TB following a treatment failure. The distribution of NTM in low resource countries with high burden TB prevalence remains unknown. Meanwhile, cost information for the management of NTM in North America is based on data published in four available studies. These studies offered a picture of direct medical costs for the outpatient management of NTM. No cost data is available from Europe, Australia, or developed countries in Asia. In the developing world, no data measuring cost or economic impact of NTM infection exists. This lack of information exists alongside the mountains of published literature describing most every aspects of cost for TB in various countries including the industrialized west as well as developing countries in Africa, Asia and Latin America, and in various care settings. 


\section{CHAPTER 3: RESEARCH METHODS}

The rising cost of healthcare is an important field of interest in most every economy as balancing resource constraints with demand has become a common challenge in both developing as well as industrialized countries. Understanding drivers of cost whether they be related to incidence or high treatment and technology costs has also become critical in resource management. Further, the presence of trends in cost implies similar trends in disease incidence and associated management costs, which has important implications for scientific interest and policy interventions. Given the lack of information available in peer reviewed published literature and national databases, Nontuberculous mycobacterium (NTM) disease remains an emerging field of research as little information is available regarding epidemiology and cost in virtually every care setting. Despite this, the importance of NTM as a medical problem and cost driver remains undocumented. Meanwhile, a myriad of data is available for Mycobacterium tuberculosis (TB) that qualifies this disease as a worldwide public health crisis, especially in select developing countries.

This study was designed to add to the small knowledge base of 
NTM, most specifically the cost and economic impact of this emerging public health challenge. The cost and trends in cost of pulmonary NTM were evaluated in the context of similar data that was ascertained for pulmonary TB for hospitalized patients in the US over a long study period, from 2001 to 2012 . Additionally, this study sought to better understand demographic features of this disease as well as define and compare, and contrast social determinants of mycobacterial infection using national population level data. Finally, clinical measures were recorded and evaluated comparing the impact of morbidity and mortality of pulmonary disease caused by NTM and TB.

\section{Study Goals}

The primary goal of this study is to conduct a comprehensive evaluation of the costs of care for pulmonary TB and NTM in US in-patient hospital settings using national data during a long study period beginning in 2001 through 2012 . The secondary goal of this study is to document cost data as well as identify important trends that may provide insight into future projections and challenges, as well as document the similarities and differences between these two diseases in demographic features, socioeconomic status and clinical outcomes.

\section{Specific Aims}

This study has two very specific aims. The first aim is to provide an accurate cost comparison of pulmonary disease caused by NTM and TB. The second aim is to identify and compare trends in cost, demographic 
features and clinical outcome data of hospitalized patients with pulmonary NTM and TB.

\section{Hypothesis}

Current published medical literature provides significant insight into the epidemiology and cost of managing TB from various care settings all over the world. Only a small percentage of this data is available for NTM infection, most of which is confined to care settings in North America. Based on published findings for both diseases, the expected results from this study include the identification of increasing rates of infection and related costs associated with the management of pulmonary NTM infection alongside flat or declining rates of pulmonary TB infection for hospitalized patients in the US. Additionally, characteristic patient demographics for pulmonary TB infection including young males of lower socio-economic status as well as older females of higher socio-economic status for pulmonary NTM infection are expected to be found following review of this population-based national dataset.

An alternate hypothesis is NTM rates and costs are represented by only a mild increase, which might be explained by the small amount of data currently available representing a sample that is not characteristic of population level national data. Another explanation might be the limitation of scope in this study in which only hospitalized patients were considered, or that only select NTM pathogens could be included. 
Regardless of outcome, important implications for future research and policy implications can be extrapolated, including the continued worldwide investment in TB control and the increase in resources required for NTM control and research that may lead to better detection and appropriate treatment in all care settings.

\section{Methods}

This is a retrospective, population based comparative study using data obtained from the National Inpatient Sample (NIS) available from the Agency for Healthcare Research and Quality's Healthcare Cost and Utilization Project (HUCP). NIS is the largest inpatient healthcare dataset in the US providing national estimates of hospital costs stratified by disease. NIS collects data from every state participating in HCUP, which includes more than $95 \%$ of the US hospitalization data. ${ }^{321}$ All patients with a principal diagnosis of pulmonary NTM due to $M$. avium, $M$. interacellulare and $M$. kansasii and pulmonary TB who were discharged from the hospital during 2001 through 2012 were included in this study. This study was submitted to the University of Louisville Institutional Review Board, where it was determined that the data reviewed in this analysis did not meet the definition of human subjects and did not require a review from the ethics board in order to proceed. The outcome letter for IRB study submission number 16.0828 has been included in the appendix.

\section{Study definitions}

This study includes both disease related definitions as well as 
geographical features, payers and other socio-economic indicators and clinical measures. Pulmonary NTM was defined by any hospital discharge associated with principal diagnosis of an International Classification of Diseases, $9^{\text {th }}$ Revision, Clinical Modification (ICD-9) code 031.0. This diagnosis code includes pulmonary infection caused by $M$. avium, $M$. interacellulare and $M$. kansasii. The Principal diagnosis was defined as the main reason for admission. The regions of the US were classified into four zones recognized by Bureau of the Census as Northeast, Midwest, South and West as outlined below in Table 12.

Table 12: Census Bureau Classification of the US by zone

\begin{tabular}{|c|l|}
\hline Zone & \multicolumn{1}{|c|}{ States } \\
\hline Northeast & $\begin{array}{l}\text { Connecticut, Maine, Massachusetts, New Hampshire, New } \\
\text { Jersey, New York, Pennsylvania, Rhode Island, and } \\
\text { Vermont }\end{array}$ \\
\hline Midwest & $\begin{array}{l}\text { Illinois, Indiana, lowa, Kansas, Minnesota, Missouri, } \\
\text { Michigan, Nebraska, North Dakota, Ohio, South Dakota } \\
\text { and Wisconsin }\end{array}$ \\
\hline Wouth & $\begin{array}{l}\text { Alabama, Arkansas, Delaware, District of Columbia, } \\
\text { Florida, Georgia, Kentucky, Maryland, Mississippi, } \\
\text { Louisiana, Tennessee, North Carolina, Oklahoma, South } \\
\text { Carolina, Texas, Virginia, and West Virginia }\end{array}$ \\
\hline $\begin{array}{l}\text { Alaska, Arizona, California, Colorado, Hawaii, Idaho, } \\
\text { Montana, Nevada, New Mexico, Oregon, Utah, } \\
\text { Washington, Wyoming }\end{array}$ \\
\hline
\end{tabular}

Several variables were defined, collected, and analyzed in this study related to disease severity, demographics and socio-economic status. Hospital length of stay was defined as the number of days the patient remained in the hospital. Payer was classified by the type of insurance coverage carried by the patient or lack thereof, which included 
Medicare, Medicaid, Private insurance (Blue Cross, commercial carriers, and private HMOs and PPOs), uninsured which includes an insurance status of "self-pay" and "no charge", and Other which includes Worker's Compensation and other government payers. Median income was defined as the average household income of patient zip code of residence and compared with national median income for the same year. HCUPnet used value ranges of the average household income itemized by year. ${ }^{322}$

Table 13: Annual ranges for calculation of median income by zip code

\begin{tabular}{|c|c|c|}
\hline Year & Low & Middle or Above \\
\hline 2003 & $\$ 1-35,999$ & $\$ 36,000+$ \\
\hline 2004 & $\$ 1-35,999$ & $\$ 36,000+$ \\
\hline 2005 & $\$ 1-36,999$ & $\$ 37,000+$ \\
\hline 2006 & $\$ 1-37,999$ & $\$ 38,000+$ \\
\hline 2007 & $\$ 1-38,999$ & $\$ 39,000+$ \\
\hline 2008 & $\$ 1-38,999$ & $\$ 39,000+$ \\
\hline 2009 & $\$ 1-39,999$ & $\$ 40,000+$ \\
\hline 2010 & $\$ 1-40,999$ & $\$ 41,000+$ \\
\hline 2011 & $\$ 1-38,999$ & $\$ 39,000+$ \\
\hline 2012 & $\$ 1-38,999$ & $\$ 39,000+$ \\
\hline
\end{tabular}

\section{Study variables}

Several variables in this study were evaluated; including age group, gender, region, payer, median income according to zip code, hospital 
length of stay in days, mean hospital charge per admission, mean aggregated charge, total aggregated charge, and in-hospital deaths.

\section{Statistical Analysis}

The statistical significance of observed trends of NTM and TB national hospital costs were calculated using Poisson log-linear regression. National hospital costs for NTM and TB were projected in relation to health care inflation for each year. Expected mean aggregated charge $(E a C)$ was calculated based on health care inflation using the equation, $(E a C=H c i \times O a C)$, in which $E a C$ is expected aggregated hospital charge, $\mathrm{Hci}$ is health care inflation percent for each year $(\mathrm{Hci}=$ total aggregated hospital charge for a specific year divided by the total aggregated hospital charge for prior year $\times 100$ ) and $\mathrm{OaC}$ is the observed aggregated charge for each medical condition. A regression model was applied to test the correlation between observed and projected aggregated hospital charges, otherwise known as national hospital costs. All statistical analyses were performed using SPSS statistical software (version 21.0, IBM Corporation)

\section{Results}

This study included an evaluation of $36,484,846$ hospital admissions in the US from 2001 through 2012. A total of 20,049 hospital discharges were reported for pulmonary NTM and 44,370 for pulmonary TB in the US from 2001 through 2012. Using national data, this study measured numerous variables including demographic, cost and clinical 
factors impacting the distribution of disease in the US. The findings from this study have important implications that both validate and expand the findings from other research.

\section{Demographic findings}

This study sought to explore demographic features of mycobacterial diseases in the context of new findings as well as published literature. A number of demographic features of patients with pulmonary NTM and TB were measured including age, gender, payer status and estimated household income. Demographic features of both diseases have been previously described relative to clinical and socio-economic importance, and the results of this study validated previous findings.

The variation of age distribution for pulmonary mycobacterial diseases is well described in the literature. These variations were also noted in the results of this study. The majority of pulmonary NTM patients were between 65 and 84 years old $(48.5 \%)$, while $70 \%$ of TB patients were younger than 65 . These findings were consistent with other studies as the association of NTM with older patients and TB with younger patients is well described. When evaluating the over 65-age group specifically, a statistically significant difference was found between the NTM and TB group $(P=0.0001)$ as well as the very elderly $(+85)$ $(\mathrm{P}<0.00001, \mathrm{OR}=2.83)$. TB was also more common in pediatric populations than NTM, with $5.1 \%$ of hospitalized patients with TB 
occurring in children 17 years and younger versus $1.2 \%$ of the population of hospitalized patients NTM occurring in children. The table below illustrates the age distribution for each disease.

Table 14: Age distribution of pulmonary NTM and TB

\begin{tabular}{|l|l|l|}
\hline \multicolumn{1}{|c|}{ Age Group } & \multicolumn{1}{c|}{ NTM (\%) } & \multicolumn{1}{c|}{ TB (\%) } \\
\hline$<17$ (pediatric) & 1.8 & 5.1 \\
\hline $18-44$ & 7.6 & 36.3 \\
\hline $45-64$ & 31.3 & 33.8 \\
\hline $65-84$ (elderly) & 48.5 & 20.7 \\
\hline$>85$ (very elderly) & 10.8 & 4.1 \\
\hline
\end{tabular}

The distribution of disease by gender demonstrated statistically significant differences between NTM and TB. As described across studies, the relationship between TB and males along with that of NTM and females are well described in the currently available medical literature. Pulmonary NTM was significantly higher in females than pulmonary TB $(\mathrm{P}<0.00001)$ and pulmonary TB is much higher in males than females. See the table below for the distribution of NTM and TB by gender. 
Table 15: Gender Distribution of pulmonary NTM and TB

\begin{tabular}{|l|l|l|}
\hline \multicolumn{1}{|c|}{ Gender } & \multicolumn{1}{c|}{ NTM (\%) } & TB (\%) \\
\hline Male & 44 & 67 \\
\hline Female & 56 & 33 \\
\hline
\end{tabular}

One particularly interesting finding of this study is the geographical distribution of TB and NTM appear almost identical. Both diseases were most common in the region defined as the South, representing $43 \%$ of cases for both diseases. One explanation for this finding is that warm and humid climates may facilitate the transmission patterns of both TB and NTM similarly. The next most common region was the West, which is also characterized by a warmer climate as compared to other regions in the US. A total of $22 \%$ of NTM cases and $23 \%$ of TB cases resided in the West. The Northwest represented the third most common region for NTM and TB at $19 \%$ and $21 \%$, respectively. Both diseases were least common in the Midwest. These data indicate that all pathogenic mycobacterium species may be impacted by the same environmental factors that drive disease transmission. See the table below for the distribution of disease by geographical zones. 
Table 16: Geographical distribution of pulmonary NTM and TB

\begin{tabular}{|l|l|l|}
\hline \multicolumn{1}{|c|}{ Region } & \multicolumn{1}{c|}{ NTM (\%) } & \multicolumn{1}{c|}{ TB(\%) } \\
\hline Northwest & 19 & 21 \\
\hline Midwest & 16 & 13 \\
\hline South & 43 & 43 \\
\hline West & 22 & 23 \\
\hline
\end{tabular}

Payer status was evaluated for TB and NTM admissions and the findings had several important implications. The most significant difference in payers noted in the two groups was Medicare, representing $61 \%$ of pulmonary NTM inpatient costs as compared to only $24 \%$ of the hospital costs of subjects with pulmonary TB $(P<0.00001)$. Given that NTM is associated with disease in older populations, the discovery that Medicare was the primary payer for NTM hospitalizations was expected. Medicare comprising $24 \%$ of TB admissions was higher than expected, but following reconciliation with the age distribution findings in this study these results were consistent with other findings from this study. One explanation with the higher than expected percentage of TB patients from this age group may be a result of the baby boomer cohort occupying a large proportion of the population, which may amplify the distribution of less common diseases in this age group. The association of TB with Medicaid and the uninsured, however, was fully expected given that these 
payer statuses are strong markers for the poor, immigrants and other vulnerable populations. The stronger association between NTM and private insurance was expected, implying a higher socio-economic association with NTM infection as reported in multiple studies. See the table below for the distribution of disease by payer status.

Table 17: Payer status of pulmonary NTM and TB

\begin{tabular}{|l|c|c|}
\hline \multicolumn{1}{|c|}{ Payer } & NTM (\%) & TB (\%) \\
\hline Medicare & 61 & 24 \\
\hline Medicaid & 10 & 23 \\
\hline Private insurance & 22 & 19 \\
\hline Uninsured & 5 & 25 \\
\hline Other & 2 & 9 \\
\hline Missing & 2 & 3 \\
\hline
\end{tabular}

For the purposes of this study, household income was defined by the average annual income of the zip code where the patient resided according to hospital admission records. Income levels were defined by two variables: low and average or above. These averages were adjusted annually based on inflation as outlined in Table 13. Zip code analysis confirmed the association of NTM and higher socio-economic status as $72 \%$ of NTM patients were defined as middle income or above. This finding was in contrast with the demographics of patients with TB in which only $55 \%$ were considered average or above. Only $26 \%$ of pulmonary NTM patients had a household income that was considered low as 
compared to $41 \%$ for patients with pulmonary TB $(P=0.0246, O R=0.51)$.

These findings are consistent with those from other reports. See the table below for disease distribution by median income based on zip code.

Table 18: Median income by zip code in patients with pulmonary NTM and TB

\begin{tabular}{|l|l|l|}
\hline Median income for Zip Code & NTM (\%) & TB (TB\%) \\
\hline Low & 26 & 41 \\
\hline Middle or Above & 72 & 55 \\
\hline Missing & 2 & 3 \\
\hline
\end{tabular}

Cost findings

Perhaps the most important finding of this study lie in the cost figures collected over the duration of eleven years, beginning in 2001 and continuing through 2012. First, the total hospital cost for all conditions recorded during the study period was $\$ 1,337,939,745,325$. Pulmonary mycobacterial diseases accounted for a total of $\$ 2,981,880,609$, which represents $0.22 \%$ of all hospital costs during the study period. Hospital cost due to pulmonary NTM was found to be $\$ 903,767,292$, which represents just over $30 \%$ of mycobacterial diseases diagnosed during the study period. Hospital cost due to pulmonary TB was calculated at $\$ 2,078,113,317$, which represents nearly $70 \%$ of mycobacterial diseases recorded during the study period. See the table below for an illustration of hospital cost due to mycobacterial diseases from 2001 to 2012 . 
Table 19: Hospital charge due to mycobacterial diseases

\begin{tabular}{|l|l|l|}
\hline Indication & Cost (\$) & Mycobacterial Diseases (\%) \\
\hline NTM & $903,767,292$ & 30.3 \\
\hline TB & $2,078,113,317$ & 69.7 \\
\hline
\end{tabular}

The average hospital charges per admission were similar for both NTM and TB. The mean aggregated charge describes the actual average annual hospital charges for both diseases, as opposed to the expected mean aggregated charge, which is a multiplier of actual hospital charges and healthcare inflation. The expected mean aggregated charge was lower for both diseases than actual charges, which implies that trends for both diseases did not meet expectation in charges during the study period. However, these values are annual averages and do not reflect trends observed for both diseases during the study period. Also listed below is the mean aggregated collection. This figure is estimated based on assumed rates of collection for each payer. These assumptions are based on industry averages which include the following: Medicare is $45 \%$, Medicaid is $35 \%$, private insurance is $55 \%$, uninsured is $20 \%$, and other and missing are estimated at $30 \%$. The overall average collection rate for NTM was estimated at $45 \%$ and for TB this rate was estimated at $37 \%$. Please note that actual figures are not available as collection data are considered proprietary contractual information. 
Table 20: Cost figures for patients with pulmonary NTM and TB

\begin{tabular}{|l|l|l|}
\hline \multicolumn{1}{|c|}{ Variables } & \multicolumn{1}{|c|}{ NTM } & \multicolumn{1}{c|}{ TB } \\
\hline $\begin{array}{l}\text { Mean hospital charge per admission } \\
(\$)\end{array}$ & 47,120 & 48,332 \\
\hline $\begin{array}{l}\text { Mean aggregated charge }(\$) \\
(\text { OaC) }\end{array}$ & $80,886,935$ & $246,398,995$ \\
\hline $\begin{array}{l}\text { Expected mean aggregated charge }(\$) \\
(\text { EaC) }\end{array}$ & $85,005,570$ & $282,501,149$ \\
\hline $\begin{array}{l}\text { Estimated mean aggregated } \\
\text { collections (\$) }\end{array}$ & 21,322 & 17,859 \\
\hline Effective collection rate $(\%)$ & 45 & 37 \\
\hline
\end{tabular}

Trend analysis in this study reflects a different result. National hospital charges for pulmonary NTM increased at a statistically significant rate each year $(P=0.001)$. A linear regression analysis demonstrated a high degree of correlation between NTM observed and projected national hospital costs $(R=0.938, P<0.001)$. This finding predicts that NTM is on an upward trajectory that will likely continue to increase in coming years. However, no such correlation between observed and projected national hospital costs was found for pulmonary TB $(R=0.284, P=0.372)$. In fact, TB rates of infection appeared to increase, but did so at a rate less than healthcare infection. Annual averages were likely impacted by movement early during the study period that did not maintain over time. See the figure below for a representation of cost trends for mycobacterial diseases relative to health care inflation. 


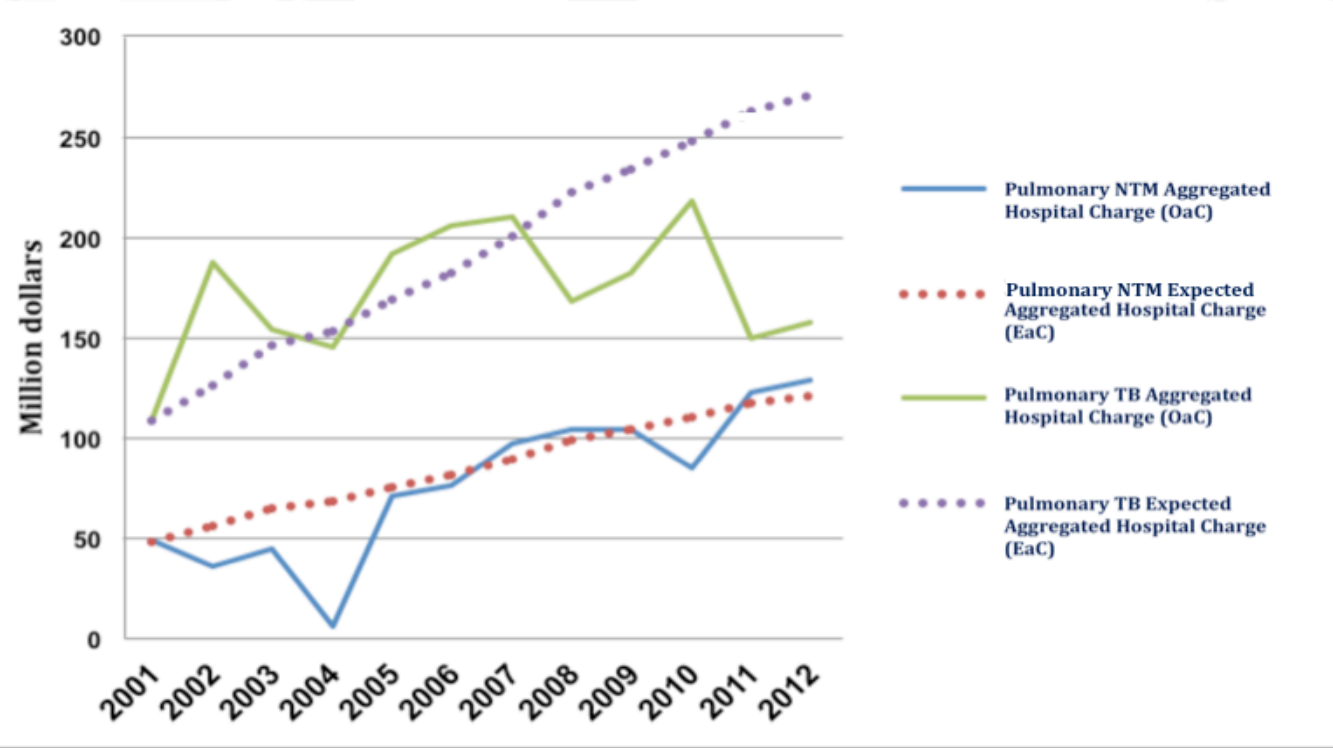

Figure 6: Mycobacterial diseases cost trends

Epidemiological trends identified NTM and TB over time also have important implications for the future of mycobacterial disease. Using admission data stratified by year for pulmonary NTM and pulmonary TB during the study period, an inverse relationship can be observed between the two causes of pulmonary mycobacterial disease. TB incidence as represented by national hospital rates is on the decline during the study period while hospitalization due to NTM is rising. See the figure below for a visual representation of the epidemiology of pulmonary NTM and pulmonary TB. 


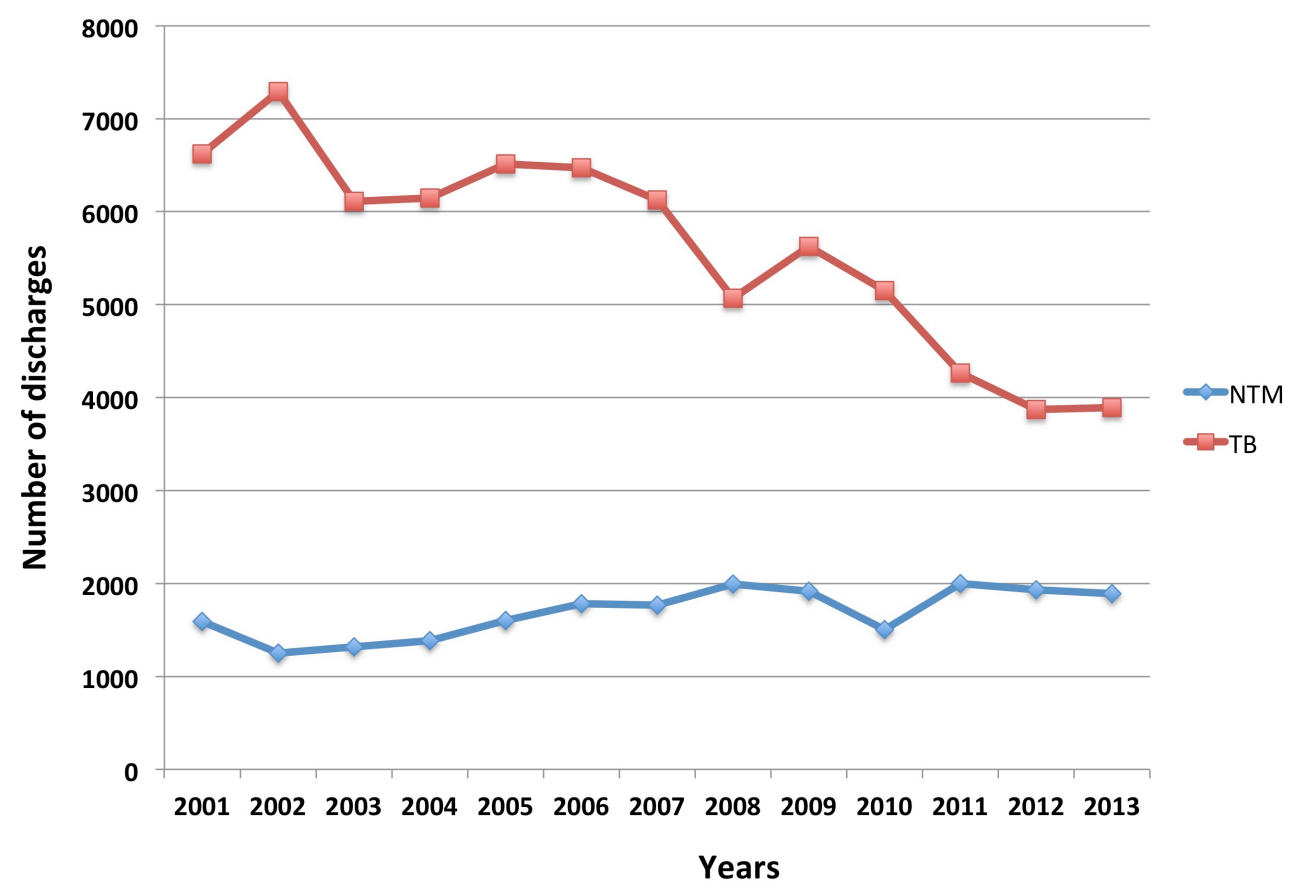

Figure 7: Epidemiological data for hospitalizations due to mycobacterial diseases in the US

Clinical Findings

Finally, clinical outcome measures were recorded as a means of understanding the impact of mycobacterial diseases relative to morbidity and mortality. A number of clinical measures were included. First, hospital length of stay was recorded for both pulmonary NTM and pulmonary TB. One interesting finding of this study was that TB patients had a longer hospital stay compared to NTM patients, TB averaging 13 days versus 9 days for NTM but the mean hospital charge per admission was only marginally higher for TB as compared to NTM. This finding 
implies an average per diem hospital charge for NTM of $\$ 5,121.74$ versus $\$ 3,580.15$ for TB. Another important outcome measure is mortality.

Mortality was recorded for all hospital admissions in which the patient died during the hospital course. The average death rate for NTM appears less in numbers than TB, but represents a higher percentage of patients. This result is reflected by the lower number of NTM infections, but demonstrates a higher likelihood of mortality. See the table below for discharge data and clinical measures for NTM and TB.

Table 21: Clinical outcomes of patients with pulmonary NTM and TB

\begin{tabular}{|l|c|c|}
\hline \multicolumn{1}{|c|}{ Variables } & NTM & TB \\
\hline $\begin{array}{l}\text { Pulmonary mycobacterial infection } \\
\text { admissions }\end{array}$ & 20,049 & 44,370 \\
\hline Length of stay in days & 9.2 & 13.5 \\
\hline $\begin{array}{l}\text { Average in-hospital mortality } \\
\text { (mean/year)(percentage) }\end{array}$ & $71(35 \%)$ & $114(26 \%)$ \\
\hline Mean hospital charge per admission $(\$)$ & 47,120 & 48,332 \\
\hline
\end{tabular}

\section{Study Strengths}

This was a population-based study that focused on the cost burden of inpatient care of patients with pulmonary mycobacterial diseases in the US between the years 2001 through 2012. Overall cost was determined to be around $\$ 3$ billion during the study period. The aggregated hospital charges for pulmonary NTM had an increasing trend that was in the same trajectory with the health care inflation. However, pulmonary TB inpatient 
costs were not increasing at the same rate as the trajectory of health care cost inflation. One could argue that the cost of managing TB during the study period was effectively decreasing.

In addition to the measurement of cost, this study also identified important demographic features of both diseases. Compared to patients with pulmonary TB, patients with pulmonary NTM were more often women. The relationship between women and NTM is well described. Also, NTM patients were older and came from areas with higher incomes, which has been found in other studies. In a 2014 review, Mirsaeidi and colleagues profiled a 70-year-old white female patient with pulmonary NTM as a typical case of NTM facilitating a discussion of NTM and females and the elderly. The authors explained that the elderly are at an increased susceptibility to NTM pathogens and noted the unique treatment challenges due to health status and comorbidities. ${ }^{323}$ Another review noted the strong association between NTM and the elderly, immunosuppressed patients of all ages, HIVIAIDS, and the availability of solid organ transplantation as an option for treating a myriad of diseases. The authors of this review note that NTM rates continue to rise, which is likely related to both population features as well as advances in detection methods. However, standards have yet to be established in management and treatment for most patients. Better evidence based recommendations based on the availability of well-designed clinical trials is critical to the future management of the disease..$^{324}$ A comparison study conducted in 
Australia compared NTM isolates obtained in 1999 and 2005 along with clinical features. This study found that NTM incidence increased from 2.2 per 100,000 in 1999 to 3.2 per 100,000 in 2005 . This study also noted important demographic changes in the disease during the study period. In 1999, NTM was more common in middle-aged men who smoke while 2005 identified the most common demographic were elderly women. ${ }^{325}$ These findings are important for several reasons. First, a recent population-based study demonstrated that females die more frequently from NTM and the majority (78\%) of NTM-related mortality occurs in the patients 55 years of age or older. ${ }^{326}$ The association between elderly females and mortality were also confirmed in a study presented at the 2016 IDSA meeting. ${ }^{327}$ Other demographic characteristics are also well described. In a sample collected from Medicare Part B of patients with pulmonary NTM between the years 1997 and 2007, Adjemian and colleagues showed a relationship between the prevalence of disease in counties in the US with a higher median household income. This study also identified the geographical distribution of disease using cluster analysis, noting that counties in warm areas like Louisiana, Florida and Hawaii were at a higher risk for pulmonary NTM as compared to counties in Rhode Island, Michigan, Minnesota and West Virginia. ${ }^{328}$ The findings in this study were consistent with those available in the peer-reviewed literature. 


\section{Study Limitations}

This study measured hospital charges associated with pulmonary mycobacterial diseases in the US. It is important to note that healthcare costs as represented by collections were estimated based on average collection rates per payer as actual collection rates are not available because this data is considered proprietary contractual information. In addition to direct medical costs having been estimated, this study failed to capture a complete picture of economic impact as most every cost study does. The full economic burden of mycobacterial disease, as is the case in almost every chronic disease, is very difficult, if not impossible, to measure. In addition to the cost of delivering direct medical care in the inpatient and outpatient setting, total cost involves many other financial and human costs. These costs may include lost wages, travel expenses and opportunity costs of the sick, the cost to administer public health programs including surveillance and prevention, and the economic impact on the family and social network of those receiving care for the disease. It is important to note that the measure of total economic cost for any disease is outside of the scope of most studies.

Another major limitation of the study was the use of ICD-9 billing codes as the search term for the database. ICD-9 coding in this study has several important limitations. First, while more than 150 species of NTM have been identified as causes of disease in humans, ICD-9 billing only itemizes $M$. avium, $M$. interacellulare and $M$. kansasii. Although these 
species represent the most common cause of disease in humans in North America, an international average has recorded these species as a cause of human disease in only half of cases worldwide. ${ }^{329}$ Therefore, it is safe to assume that this study failed to capture a significant portion of NTM cases caused by other species, which leaves many unanswered questions regarding the true cost of care for patients hospitalized with NTM disease. Another important limitation associated with ICD-9 is that this study population only includes patients hospitalized with pulmonary mycobacterial disease. TB and NTM can cause disease processes in most every part of the body including but not limited to skin and soft tissue, bone and joint, other major organs and disseminated disease. Although these cases are less common than pulmonary disease, they are more common particularly in immunosuppressed hosts and represent an important cost driver in the management of mycobacterial disease.

This study was limited to inpatient cases of NTM and TB. Mycobacterial diseases are frequently managed in an outpatient setting given the chronicity and sometimes mild symptoms, so these cases of pulmonary NTM and TB were not considered in this analysis. Further, it is not uncommon for NTM to be diagnosed in hospitalized patients following discharge given the slow growth of the pathogen in the laboratory. Therefore, patients with pulmonary NTM are assigned another billing code in these cases such as community acquired pneumonia, and were not included in this study. In the event of a readmission, cases would be 
coded correctly and only for subsequent admissions. Therefore, all cases of pulmonary NTM due to $M$. avium, $M$. interacellulare and $M$. kansasii were not included in this study. Given these important limitations, it is important to emphasize the cost of managing TB and especially NTM most likely significantly exceeds what was measured in this study. 


\section{CHAPTER 4: DISCUSSION}

The purpose of this study was to determine the cost of care of managing two important diseases in the US. Although the cost of TB has been extensively measured in a variety of care settings, this study offered another perspective of TB management cost in the US as well as identified important cost and epidemiological trends. Also, this study contributed to the emerging cost data for the more elusive group of pathogens known as NTM. Additionally, epidemiological and cost trends are identified in this study showing an increase in both. This study offers important information establishing the importance of NTM for future research. Further, the findings of this study have several important implications for public health policy.

\section{Implications for Policy}

Globalization is a contemporary construct that describes the free movement of people, information, goods and services accessed across political and physical borders that separate countries, continents, cultures, religions, and societies. Borders in the modern world are fluid as populations shift, information is shared, and economies are intertwined. Globalization also has important implications for health as global 
connectedness is demonstrated by the exposure of disease by the interaction of populations with one another and a common environment like never before, leading the epidemiology of many diseases to be transformed into global public health challenges.

Policy makers who design and evaluate local and national health policy face many challenges due to the complexities and dynamics of influence from the outside. A number of tools and frameworks are available to these experts that reduce the factors influencing a system and its drivers in order to identify opportunities to intervene and influence drivers that impact outcomes as a means of reaching program goals. Among these frameworks is the Ladder of Engagement, which offers an important decision support tool allowing policy makers to reduce complex systems to linear terms. The Ladder of Engagement utilizes both Systems Thinking and Dynamic Modeling by identifying catalysts that drive human behavior over time. Through the identification of nonlinear relationships represented by both positive and negative feedback loops, policy makers are able to isolate indicators of system behavior that represent opportunities for policy interventions that would enhance the ability of public health programs to be designed to reach policy goals.

\section{System Dynamics: Systems Thinking and Dynamic Modeling}

Critical to the process of System Dynamics is the use of two equally critical and consecutive steps: Systems Thinking and Dynamic Modeling. Systems Thinking provides the conceptual framework for establishing the 
elements used in Dynamic Modeling, ensuring that the model is representative of the system so that accurate and reliable simulations occur. ${ }^{330}$ Systems Thinking alone provides an incomplete analysis of the process because the complexity of mental models exceeds one's capacity to fully understand the implications without the process of model creation, mapping and simulation. Just as Systems Thinking is dependent on Dynamic Modeling for providing advanced simulations and experiments predicting interactions and behaviors in a system, Dynamic Modeling is equally dependent on Systems Thinking to establish the model conceptually and represent the relationships and interactions accurately so that simulations and experiments precisely reflect the dynamics of the system. ${ }^{331}$

Systems Thinking represents the first step in the process in which policy makers become oriented with the situation and capture the connection between parts and understanding their relationships to the functioning whole. Although this step is considered qualitative, it does not represent a rejection of traditional scientific views built on mechanic and linear relationships. Instead, Systems Thinking builds on these concepts with increased complexity rather than undermining them while requiring one to conceptualize systems as wholes as well as to precisely identify critical relationships and interactions that impact the whole. ${ }^{332}$

Dynamic Modeling refers to the quantitative aspect of the System Dynamics that involves the use of models and simulations to increase the 
understanding of policy makers of complex systems. The opportunities for policy makers to use Dynamic Modeling to approach complex problems are significant as modeling represents a method of understanding change in a complex and dynamic system so that the influences within can be better understood and the direction of the system can be governed. Also, mapping and simulation allows policy makers to identify potential opportunities within the system for the most effective policy interventions to optimally navigate system change. ${ }^{333}$ These experiments allow policy makers to input different policy interventions and better visualize outcomes relative to policy goals and those unrelated including unintended consequence as a means of evaluating policy prior to implementation. Although the purpose of constructing these models is not to necessarily to allow policy makers to make predictions into the future, modeling does have the potential to shed insight into how behavior and interactions over time will impact the system. ${ }^{334}$

\section{The Ladder of Engagement}

System dynamicists possess a myriad of backgrounds and expertise as notable in the Center for Interdisciplinary Excellence in System Dynamics. This diversity in background has led to the development of a collaborative model for approaching complex problem solving. The Ladder of Engagement was developed and refined as a means of progressively and comprehensively understanding and describing complex interactions and behaviors in a system. The model 
consists of three separate but dependent levels: knowledge, understanding, and influence.

\section{Knowledge}

Critical in the knowledge segment of the Ladder of Engagement framework is knowing how a system behaves. Therefore, defining the problem and developing an accurate and representative problem statement is the first step in the process. This step is critical in identifying contributing factors to a problem situation and conceptualizing the relationship. Also, the mapping process key in Dynamic Modeling is initiated as positions within the system, or stocks, are identified and defined relative to outcome stocks. When mapping global public health problems, the most critical stocks in a system is death, and the positions within the system that flow directly into death may represent the problem situation.

\section{Understanding}

The next step in the Ladder of Engagement Framework is understanding. This step involves the identification and evaluation of leverage points in the system that can potentially drive behavior. This too calls for conceptualizing relationships as part of the Systems Thinking process as well as building on the established model. During this step, positions within the system are identified and represented by their role in affecting behaviors and outcomes within the system over time. These positions are represented in the model as stocks. Stocks that have an influence on a 
desired outcome may identify opportunities in which this influence can be magnified. Stocks that have an undesired outcome are identified as leverage points. Opportunities to reduce the flow into an outcome stock of death exist in these positions.

Influence

This is the final step in the analysis in which policy makers determine whether the identified opportunities in the system are modifiable through policy change. A systems dynamicist would likely employ the simulation phase of the Systems Dynamics process after policy development but prior to implementation to determine whether policy outcomes met policy goals and to identify resulting unintended consequences using the stock and flow modeling process. ${ }^{335}$

\section{Systems Dynamics and Public Health}

Systems Dynamics has been used to address public health issues since the 1970s. Among these issues include disease epidemiology for both chronic diseases and infectious diseases, substance abuse including tobacco as well as illicit drugs, the movement of patients through the healthcare system including from emergency care to long term care facilities, healthcare resource capacity and delivery as conducted by HMOs, and the interactions between healthcare capacity and public health capacity in relation to specific disease epidemiology. ${ }^{336}$ The identification of public health issues appropriate for Systems Dynamics tools are 
relatively easy to identify and, often times, the catalysts are likewise easy to isolate. ${ }^{337}$

Systems Dynamics tools are being used in the surveillance of diseases in order to identify emerging pandemics and form response strategies. The Centers for Disease Control and Prevention (CDC) possesses a well-established role in disease surveillance. Using their proprietary system called BioSense, the CDC is able to feed epidemiological data into the system from local, national and even international sources to identify emerging and predict existing outbreaks of influenza. This software allows the CDC to identify outbreaks sooner, in which case the organization is able inform and mobilize local health departments as well as policy makers so that the appropriate action can be taken to prevent further disease spread. In this example, the CDC identified a potential influenza pandemic as a problem situation due to the 1918 outbreak of the Spanish flu as well as the anticipated avian flu pandemic. Mapping the problem and understanding epidemiological outbreak patterns associated with influenza led to the development of this simulation software to deliver predictions in real time. Another example is the Laboratory Response Network, also managed by the CDC, which relies on an integrated system of laboratories that collect and feed data regarding communicable diseases and bioterrorism into a simulation program to allow for the rapid identification of a disease outbreak or acts of bioterrorism. Additionally, the software provides decision support in the 
formation of a response plan. ${ }^{338}$

Despite the potential utility of Systems Dynamics to public health, many public health interventions in the US fall short of policy goals due to the inability of policy makers to approach problem situations holistically. The reason for this lies in the fragmented organizational and funding structure of public health. As a result of these institutional barriers, public health is unable to assess and implement policy that addresses system wide catalysts. Instead, policy is enacted in increments over time and often lacks the foresight and scope to address future complex problems. Therefore, public health is particularly in need of complex analytical tools to accurately and comprehensively address population level needs in the present and anticipate future needs by identifying and understanding trends over time. Public health has seemingly settled for an incomplete analysis including only the conceptualization of complex problems, and this approach has proven incomplete with the absence of simulation studies. ${ }^{339}$ Although Systems Dynamics is particularly well suited for public health programs and interventions, institutional constraints in the US have acted as hindrances to the ability of state and local public health departments to explore System Dynamics tools in complex problem solving.

\section{Mycobacterium Tuberculosis}

Public health policy for Mycobacterium tuberculosis (TB) in the US has focused on prevention through screening, contact tracing and early 
treatment. Despite institutional challenges, TB policy in the US has been very successful in preventing the transmission of disease within local populations. These results have been shared in other industrialized nations. Also, this preventative approach utilized in North America and Western Europe has been validated by systems dynamic modeling. In a 2011 Canadian study, a dynamic model was used to evaluate contact tracing and its role in the prevention of TB transmission. In this model, the population was categorized based on risk status and health status and placed into one of ten stocks. However, individuals who had been identified as an at risk contact under investigations through contact tracing were placed in the eleventh stock due to possible disease exposure. The model illustrates transitions between stocks flowing in the upper part of the diagram downward to demonstrate the change in status relative to risk of infection over time. Tian and researchers ran the model in this study, and their data supported the role of contact tracing in a theoretical closed community. However, the findings of this study implied the expansion of the program currently being used in Canada could yield diminishing returns. See the figure below for a visual representation of the model used in this study. ${ }^{340}$ 


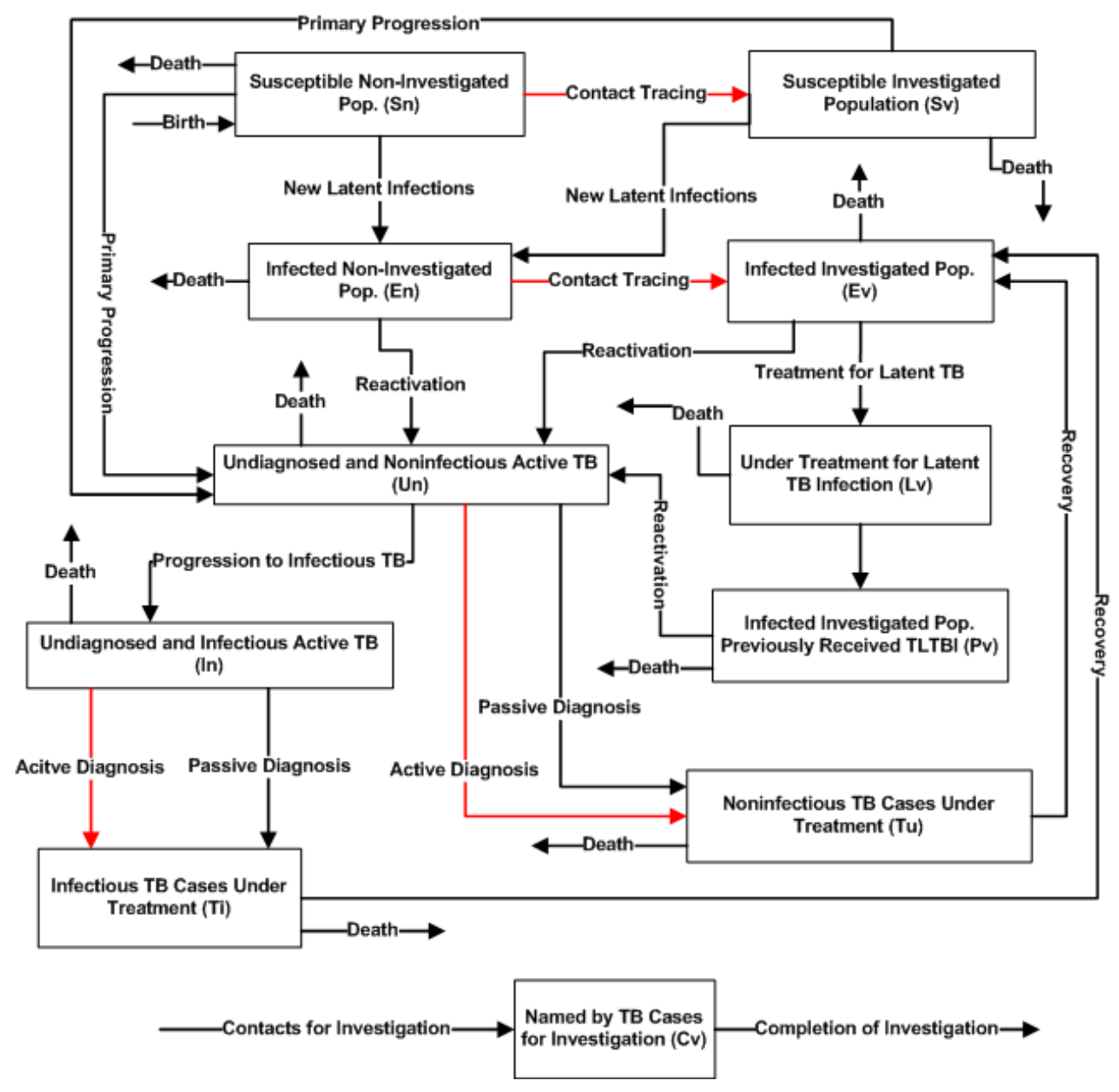

Figure 8: TB contact tracing model

Despite the successes of TB control in many countries, TB remains an important cause of disease in select populations worldwide. Among the consistent features of TB is its association with poverty. Whether this relationship is observed in overall wealthy societies in which poor populations are disproportionately affected or poor countries in which the average population of impoverished citizens is affected, where one finds poverty is likewise where one finds TB. The financial and economic burden in a variety of these settings has been well described in the 
literature. However, policy challenges are looming in some care settings while others have had considerable success.

In the developing world, a significant amount of research has been conducted on the economic burden and overall ramifications of disease to countries and its citizens in these care settings. According to a systematic review, the average cost burden for those accessing TB treatment found across studies was as follows: $20 \%$ direct medical care, $20 \%$ indirect medical care, and $60 \%$ lost wages. The $\mathrm{WHO}$ argues that programs designed to meet the needs of patients should address economic costs including the provision of direct costs, indirect costs, as well as lost wages of those with disease and their families and caretakers. ${ }^{341,342}$ Policy research agrees that without full economic support, TB patients may not be able to access TB treatment resources or complete treatment regimens. ${ }^{343}$ The impact of not ensuring patients have the resources and support to have access and be compliant with treatment on a global scale is arguably more significant than its cost. The identification of TB in populations coupled with appropriate treatment hurdles cost exponentially by preventing further transmission and undermining the facilitation of drug resistance.

Systems dynamics can be applied to TB to this scenario using the Ladders of Engagement method. In the underdeveloped world, two major undesirable outcomes exist. The first is death, and TB as a cause of death is an important medical problem in most every country. The other 
undesirable outcome is drug resistant TB, which occurs through transmission from one person to another or occurs following inappropriate treatment. ${ }^{344}$ This initial model represents knowledge of the situation. Stocks that feed into these outcomes are treated and untreated TB, which represent an important opportunity for intervention according to most studies. Assuring that diagnosed patients receive treatment is critical to TB control in most resource scarce high burden countries. Not to say that the stock of patients who adhere to TB treatment do not have poor outcomes, but the stock that does not receive and complete treatment runs the highest risk for death as well as developing drug resistant disease. This represents the second step, which establishes an understanding of the situation. Barriers to treatment are mostly cost, and it is important to note that this population is usually unable to absorb any costs including direct medical care, indirect as well as lost wages without extreme hardship. According to the literature, the total average patient costs in all care settings was equivalent to approximately one year of individual income. ${ }^{345}$ Therefore, the ability to access treatment represents the most critical barrier for this population and characterizes the most important opportunity for policy intervention. Identifying opportunities as well as evaluating policy proposals prior to implementation occurs during this step. It is also important to note that policy makers should fully understand the impact of policy relative to unmet needs and policy goals, in addition to the identification of unintended policy outcomes that may 
influence the quality of the intervention.

TB control efforts by public health have been successful in the developed world including the US. However, these measures have only been effective in local populations as $64.6 \%$ of TB cases occurred in populations born outside the US. ${ }^{346}$ In the US, death secondary to TB is not as common as compared to outcomes in developing countries but TB policy has a much stronger emphasis on prevention. Therefore, undesirable outcomes for policy makers include the disproportionate rate of infection in immigrant populations primarily from high TB burden countries. The major contributing factor in the US is immigration policy itself, which also represents an important opportunity for policy intervention given that the target group can be screened for active and latent disease during the immigrations process. According to policy researchers, universal screening for active disease using chest radiography has been widely disproven due to high cost and low yield. ${ }^{347}$ Therefore, targeted screening has been proposed that limits the time and expense of chest radiography to migrants from high burden TB countries following face to face interviews in which risk profiles and clinical information are correlated. ${ }^{348}$ In this case, the stock of TB would be decreased with targeted screening more effectively and a lower cost than universal screening. However, using the immigration process as a segue to TB control in select migrant populations limits the reach of TB control to legal migrants. ${ }^{349}$ Outreach to TB control in illegal immigrant populations 
could be accomplished through one of several methods. First, a reduction in illegal immigration through increased border control could effectively reduce the number of new cases of TB in the US. Another option is to amend immigration policy so that immigrants may enter the US legally and therefore be subject to the same processes as other migrants. Both of these interventions would require widespread political support coupled with action from legislatures, which is unlikely given the controversial nature of immigration policy in the US. Another option is to design an outreach program managed by public health specifically to reach illegal immigrant populations. For this program to be successful, public health would be responsible for the identification of and significant relationship and trust building with invisible populations of illegal immigrants who often wish to remain invisible due to fear of discovery and deportation. For illegal immigrants, the stock of TB would be decreased by public health outreach to these populations. Policy research generally agrees that to tackle the issue of TB in foreign-born populations, a complex multi-system approach is necessary to targets at risk populations through various approaches.

\section{Nontuberculous Mycobacterium}

Having only been discovered in 1959 , NTM is an emerging public health problem for which local and global policy has not been established. ${ }^{350}$ Despite this, NTM is an increasingly important cause of morbidity and mortality in the US and possibly the world. ${ }^{351}$ In fact, 
evidence suggests that NTM is a more common cause of death in the US than TB. ${ }^{352}$ Key features of the disease make the formation of policy particularly challenging. First, clinical signs and symptoms result in the disease being easily confused with TB in many health care settings, ${ }^{353}$ but epidemiology and modes of transmission are absolutely different. Therefore, effective policy that addresses NTM infection must be unique and not simply a modification of effective TB surveillance and control.

In the developing world, NTM has been barely described. Therefore, the epidemiology of NTM disease in resource constrained care settings has not been published. Some data indicate that NTM infection may be incorrectly labeled in many care settings in the developing world as drug resistant TB infection. TB drugs being used incorrectly to treat NTM infection represents inappropriate antibiotic use and has important long-term consequences for the future of antibiotic effectiveness and short-term costs. ${ }^{354,355}$ It is also possible that in some cases, people in the developing world are co-infected with both diseases. In these cases, initiating TB treatment only partially treats one infection leading clinicians to consider these patients a treatment failure and continue anti-TB drugs, or modify to second tier for supposed drug resistant TB. Both of these scenarios also represent inappropriate antibiotic use as well as over utilizing healthcare resources. It is also important to note that in the developing world, the epidemiological differences of affected populations and the geographical distribution of disease has not been established. 
Therefore, these factors cannot be used for diagnostic support in the developing world. Only through the use of laboratory techniques can NTM in this care setting be accurately diagnosed.

The undesired outcomes for NTM are undiagnosed and misdiagnosed NTM disease. When patients are being evaluated for TB, it is at this point that policy makers would suggest an intervention in which patients are diagnosed using laboratory methods that can accurately differentiate between different species of mycobacterium disease assuming access. This is particularly challenging in some parts of the world in which laboratory methods are antiquated, infrastructure is subject to supply shortages including water and electricity, or TB is diagnosed without the use of laboratory methods in particularly resource constrained care settings. ${ }^{356}$ In these cases, the introduction of enhanced laboratory methods to identify cases of NTM infection might represent the establishment of new infrastructure, which may not be possible. However, in countries with a high burden of drug resistance and HIVIAIDS, data establishing that these cases sometimes are misdiagnosed NTM could stimulate the demand for mycobacterium species typing if a significant portion of the TB control budget is being used for the treatment of drug resistant cases. Therefore, policy makers must rely on more research in the epidemiology of NTM disease in developing countries before having the ability to measure the impact of policy interventions.

NTM in the developed world is slightly more described. First, 
infection rates have been on a steady incline as demonstrated by reports in the peer reviewed medical research as well as this population-based study. ${ }^{357}$ Also, given its association with the elderly and immunesuppressed hosts, the differing epidemiology in some cases allows the clinician to better identify NTM infection amidst TB infection. ${ }^{358,359,360}$ Also, accurate microbiological detection methods have been able to tease out NTM cases with the appearance of TB with remarkable accuracy. Currently, it remains unclear whether NTM rates are truly rising or the identification of NTM species coupled with the improvement of detection rates have resulted in the incline during the study period.

Despite these questions, many researchers in the peer-reviewed literature believe NTM rates truly are increasing. Among the explanations are population features in the US and much of the developed world that are uniquely different from those in the developing world. First, the distribution of age in the US as well as many western European countries has resulted in a large segment of the population being considered elderly. ${ }^{361,362}$ As discussed, older age is an important risk factor for NTM infection. Another important risk factor for NTM infection is suppressed immune status, which may result from a number of causes in the US including HIV infection, post-transplant therapy, immune modulating therapy, and cancer treatment. This is also increasingly common in the US and is likely to also contribute to the incidence of disease. ${ }^{363,364,365,366,367,368}$ Another theory is the infrastructure of the built 
environment. NTM is primarily transmitted from environmental sources including soil and water. ${ }^{369}$ Several studies have sought to identify specific modes of transmission in patients with confirmed NTM infection by confirming the presence of the same NTM species in water samples collected from their homes. Researchers have demonstrated the pathway to transmission from water sources through the aerosolization of water from showerheads and hot tubs. NTM has also been identified in public water sources. ${ }^{370,371}$ Further, some researchers have suggested that NTM is a result of the progress of modern societies as the disease is transmitted through the public water distribution infrastructure. ${ }^{372}$ Another possible pathway for NTM infection is in the food supply. ${ }^{373}$

In the US, the undesired outcome for policymakers is NTM infection. As public health is well acquainted with employing measures to prevent the person-to-person transmission of diseases, measures to prevent disease transmission from the environment is largely uncharted territory. Although the removal of NTM species from surrounding nature sources is not possible, preventing the transmission of disease from public water and food might be an opportunity for a policy intervention.

\section{Policy Recommendations}

The purpose of this study was to better understand the emerging public health challenge of pulmonary NTM infection as presented in the context of the well-established public health challenge of the genetically similar pulmonary TB disease. Using data methodology to compare the 
cost of care and epidemiology of both diseases as well identify trends over time, this study confirmed the importance of pulmonary NTM as an increasingly critical public health challenge. This designation leaves important questions for the future of this disease as well as the implication of public health policy intervention and further research.

System dynamics can be an important decision support tool for the formation and evaluation of policy for select problem situations. Ideally, the problem situation should be clearly definable given that the relationships and catalysts have been studied and identified and the dynamic behaviors that feed the causes and effects of these relationships are well understood. Because transmission patterns for NTM disease remain elusive in the current body of medical research along with the incidence of disease in select population with identifiable risk factors seeming to occur sporadically, the cause and effect of this problem situation remain obscure. This leaves many challenges for the formation of an effective intervention using this particular decision support tool. Therefore, much more needs to be learned about NTM from both a scientific and policy perspective in order to craft effective interventions that undermine the incidence of disease in populations in the US and worldwide.

Although systems dynamics may not be suitable for the evaluation of pulmonary NTM policy given that currently available data are limited, the mapping of not well understood problem situations can reveal 
important implications for future research and the early formation of holistic policy. Mapping may lay the foundations for defining important relationships leading to discoveries being made regarding the cause and effect of important interactions. Also, opportunities for policy interventions may be revealed during this process, although in the absence of dynamic modeling they may not be testable. In select situations in which policy interventions may be suitable despite the absence of ideal confirmatory data to support its efficacy, mapping alone may be an important step in the process of forming preliminary but effective policy. Mapping may also identify what necessary data is needed to better understand a policy situation, which may be an important precursor to policy formation.

The mapping of NTM as an emerging public health problem in the US reveals important opportunities for policy makers. First and foremost, mapping demonstrates that not enough information is known about two very important aspects of NTM disease. The first is epidemiology. The distribution of NTM disease remaining unmeasured is a global problem, with the few data that exist having been pieced together from various studies that still only represents an unknown fraction of NTM cases. The mapping of NTM along with current research also reveals that little is known about clinical aspects of NTM disease. Considering that modes of exposure, transmission, and risk factors remain under described by current medical knowledge, the use of these variables in a model map only reveals to system dynamicists the information that remains 
outstanding before proceeding to the next step. In this case, understanding variables may represent key opportunities for policy and research. See the figure below for a comprehensive map that represents the known relationship between NTM with risk groups and the environment.

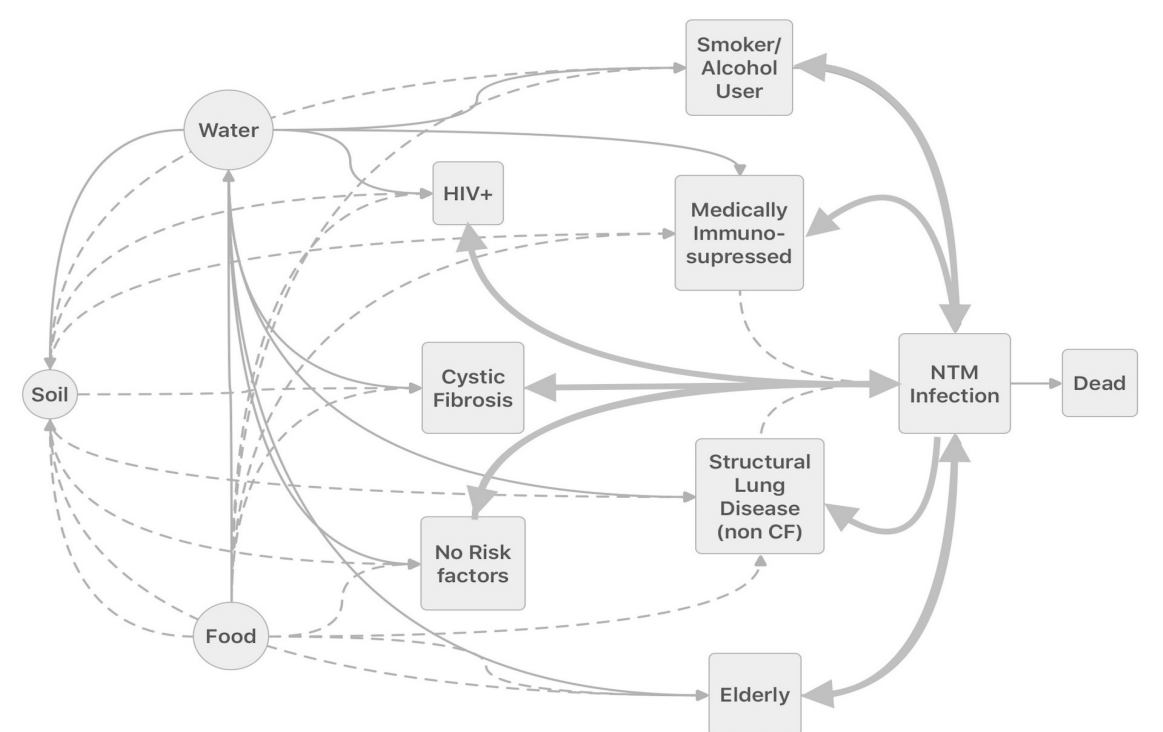

Figure 8: Mapping of NTM Transmission and Illness

Given that so little is known about the epidemiology of NTM disease, the first important policy recommendation might be in the area of disease surveillance. This might be achieved through the public health designation of NTM as a reportable disease. Public health classifies select diseases as reportable that have been designated as particularly important public health problems. In the US, diseases are reported to public health agencies in a variety of ways. For some diseases it is mandatory for each case to be submitted by paper reports or by telephone 
or even by computer, depending on the disease. Gonorrhea is an example of a disease that is reported using paper forms whereas pertussis is reportable by phone. In these cases, identifiable data are often collected in order to allow contact investigation. Other diseases are reported to public health by proving a count of diagnosed cases. Influenza is an example of a disease in which merely the number of cases diagnosed is reportable. ${ }^{374}$ All surveillance data are reported to the National Notifiable Diseases Surveillance System (NNDSS), which houses local, state, and national data managed by the CDC Division of Health Informatics and Surveillance (DHIS). The DHIS submit, process and release data from the NNDSS to all levels of public health to support surveillance and analysis of disease distribution on all levels of government. ${ }^{375}$ The purpose of disease reporting to public health agencies is to provide local, state and national surveillance of important diseases in order to identify early trends in the occurrence of disease. The ultimate goal is to maintain a constant measure of disease distribution to gain a better understanding of baseline disease occurrence, prevent outbreaks as well as to undermine the natural trajectory of future cases. ${ }^{376}$

The accuracy of national surveillance data for reportable diseases has been questioned. In a 2011 study conducted in North Carolina, researchers found that complete reporting rates varied among the eight health care systems studied from $2 \%$ to $30 \%$. Also, of the fifty-three diseases evaluated, reporting completeness rates ranged from $0 \%$ to 
$82 \%$. The discrepancy in reporting did not seem to be related to the perceived public health importance of the disease in question. Instead, diseases that were diagnosed through straight forward laboratory methods or clear clinical criteria were more likely to be reported than those that were difficult to identify clinically and/or involved multiple laboratory steps to establish a diagnosis. ${ }^{377}$ Also, physician knowledge regarding reportable diseases has likewise been questioned. A survey study of 162 emergency department physicians representing 34 states in the US indicated familiarity with IDSA guidelines for infectious foodborne pathogens, but did not demonstrate the same knowledge regarding the public health importance and reporting requirements. Given that emergency departments represent primary care form so many Americans, this finding is of increased importance. ${ }^{378}$

Recent federal legislation encouraging the use of information technology in healthcare has also sought to improve the rates at which notifiable diseases are reported. The American Recovery and Reinvestment Act of 2009 in combination with the Health Information Technology for Economic and Clinical Health (HITECH) Act has encouraged widespread adoption of electronic health records by providing federal incentive funds to providers who adopt and maintain its use. Among the components of health record technology qualifying for incentive is the inclusion of disease surveillance and electronic reporting, which is expected to not only increase the rates of reporting, but also 
reduce the number of errors and incomplete reports submitted with public health departments and the CDC. ${ }^{379} 380$ In a comparison study evaluating traditional reporting methods to the local health department with automated electronic laboratory reporting through a health information exchange, a total of 4785 reports for 53 reportable conditions were evaluated. This study found that automatic electronic laboratory reporting identified 4.4 times as many cases as traditional reporting and did so on an average of 7.9 days sooner. ${ }^{381}$ Given the widespread adoption of electronic medical records secondary to $\mathrm{HITECH}$, it is conceivable that automatic improvements in the availability of surveillance data for reportable many diseases may be an important policy outcome. However, the $\mathrm{WHO}$ notes that the reporting of some notifiable diseases on an international level do not occur for a variety of reasons US. First, some cases of disease may not be treated in a healthcare setting, but instead at home, in which case there would be no official record. Also, reportable disease may not be diagnosed properly, which would undermine reporting. In these cases, the disease in question may be difficult to diagnose or the healthcare facility may not have adequate resources to make an accurate laboratory based diagnosis. Also, reporting of notifiable diseases may be less common in under resourced healthcare settings despite the ability to make accurate diagnoses if employees are not educated or motivated to comply with reporting. Finally, a fear of economic or political consequences may result in some diseases being intentionally not 
reported. ${ }^{382}$

NTM as a reportable disease would have several distinct challenges. First, NTM is commonly confused with TB as they often present with similar clinical symptoms. Also, NTM is diagnosed through a multi-step, slow laboratory process, which current research has shown would reduce the frequency of reporting. Further, it is not uncommon for NTM to be diagnosed following hospital discharge, resulting in patients being managed for community-acquired pneumonia. In these cases, is in uncertain how disease reporting would proceed as medical coding is rarely updated. For these reasons, it is likely that designating NTM a reportable disease will still not provide a complete picture as is the case with many reportable diseases, but the designation of NTM as a reportable disease would vastly improve current available data on the disease.

Another method of improving surveillance data for NTM disease involves piecing together currently available data sources. Using national hospital data, as was the case in this study, provides one aspect of NTM epidemiology as well as the cost of care. Combining these results with outpatient data that are available in the form of searchable datasets using ICD billing codes for each payer including commercial insurers and public payers could provide a more accurate epidemiological picture. However, the collection of outpatient data would represent a laborious process to collect and analyze each individual dataset. Given that research in the 
field of NTM is likewise not being highly encouraged by the availability of abundant funding or significant scientific interest, the availability of said data that is matched with that of this study is not likely to be published. Further, the limitations of these data would be the same for this study, primarily the use of ICD-9 codes in which only three species of NTM was captured. Finally, piecing hospital data with outpatient billing with searchable billing codes would offer a much closer-to-complete picture of NTM especially if ICD billing codes were to include more species of NTM, including a designation for other common atypical mycobacterium species. The table below outlines the current billing codes allotted for NTM infection.

Table 22: ICD-10 Billing Codes for NTM Infection

\begin{tabular}{|l|l|}
\hline \multicolumn{2}{|c|}{ Atypical Mycobacteria } \\
\hline A31.0 & Pulmonary mycobacterial infection \\
\hline A31.1 & Cutaneous mycobacterial infection \\
\hline A31.2 & Disseminated MAC \\
\hline A31.8 & Other mycobacterial infections \\
\hline A31.9 & Mycobacterial infection, unspecified \\
\hline
\end{tabular}

Since the ICD-10 update, pulmonary NTM remains only applicable to infections caused by $M$. avium, $M$. intracellulare, and $M$. kansasii. Other NTM infections specified by current billing codes include cutaneous NTM, which refers to necrotizing skin and soft tissue infections caused by NTM 
organisms M. marinum and M. ulcerans. Also, disseminated disease caused by MAC is itemized alone. Other mycobacterial infections are specified to include disseminated NTM caused by M. abscessus, $M$. chelonae, M. chelonei, M. fortuitum, and M. gordonae. The final code designation applies to any other disease process caused by NTM and/or any other species of NTM. ${ }^{383}$ Although this represents an expansion of ICD-9, given the sheer numbers of NTM organisms as well as the potential for involvement of virtually any part of the body, relying on billing codes to define the presentation and distribution of NTM disease in the US might not be realistic.

Another important policy implication is, therefore, that research and scholarship in the area of NTM should be encouraged through the availability of national grants to facilitate a better understanding of this increasingly important cause of morbidity and mortality in the US. Given that NTM is estimated to be a more important disease in the US than TB by several measures, it is questionable that more data continues to be generated in the various aspects of TB infection. A study published by Raju and colleagues explains that as long as the $\mathrm{NIH}$ continues to largely ignore NTM as an important cause of human infection by supporting very few funded studies, NTM as an important cause of disease will continue to be under appreciated. ${ }^{384}$ Further, private sector interest in the treatment of most infectious diseases has been low for some time, given that research into chronic conditions for which long-term maintenance 
medications are often prescribed represents a stronger revenue model for private sector research. One important exception is HIVIAIDS, due to the chronicity of infection that results in lifetime treatment regimens for most patients. Further, political support for research in the area of HIVIAIDS coupled with the willingness of the Food and Drug Administration to fast track new therapies has also stimulated private sector interest. It is unlikely that NTM could rally similar support, but any discussion on the importance of NTM disease in the private sector could have an impact. Further, the single act of designating NTM as a reportable disease could facilitate both public and private sector interest, especially following the availability of more accurate surveillance data. 


\section{CHAPTER 5: CONCLUSION}

Mycobacterial diseases are a global health problem, representing a source of an immeasurable and significant economic burden. Describing infections caused by Mycobacterium tuberculosis (TB) and Nontuberculous mycobacteria (NTM), mycobacterial diseases represent important public health problems with significant health and economic consequences. Both TB and NTM have many clinical presentations, but the most common manifestation of disease is pulmonary. Also, both TB and NTM have a similar clinical picture along with characteristic nodules observable on lung imaging for pulmonary disease that is very distinct from other infectious diseases. Despite similarities, TB and NTM differ dramatically in a number of ways. In developing countries, NTM is more strongly associated with older hosts, particularly women, and those with underlying diseases that affect lung structure, undermine immunity, or those with medical conditions that require immune modulating therapies. TB, however, is associated with younger men, while comorbid conditions and unhealthy behaviors may also represent important risk factors. TB is also strongly associated with vulnerable populations including recent immigrants from high burden TB countries. In the developing world, the different demographic features of the two diseases are less described. 
Given that emerging research indicates that the two diseases are often confused due to the similarities in clinical appearance coupled with a lack of available diagnostic tools that accurately differentiate between the two diseases, it is understandable that in some care settings how little is known about NTM relative to TB. However, the misdiagnosis of subsequent inappropriate treatment of NTM as TB or drug resistant TB has important worldwide implications for both cost of care as well as clinical outcomes. Also, inappropriate treatment has important consequences for the future treatment of mycobacterial diseases by facilitating drug resistance.

One important similarity between the two diseases in most every care setting is the economic consequences of infection. Mycobacterial diseases caused by both TB and NTM represent serious infections that require months of treatment along with routine monitoring to ensure clinical response to treatment. Also, the risk of side effects due to extended antibiotic therapy is high and must be monitored along with treatment progress. For patients and their families, the cost of medical care coupled with the economic cost of lost wages, travel and other expenses can be devastating for many in developing countries as well as for vulnerable populations living in poverty in developed countries. Given that mycobacterial diseases have a strong association with poverty, the impact of disease on people and local economies can be catastrophic. Further, in some societies a diagnosis of mycobacterial infection can also 
have personal consequences considering the stigma of disease. This is particularly the case with TB infection as people have reported discrimination for employment, mate selection, and isolation from family and other important social networks.

In the US, mycobacterial diseases represent a unique challenge. TB in the US has been successfully controlled following effective population based public health policy interventions focused on early identification and preventions. Despite major challenges in TB care, including drug resistance and co-infection with HIV disease, rates have been stable and even decreasing in recent years in the US. In local populations, the success of TB control can be better measured as rates have declined drastically. In recent immigrant populations, the distribution of TB infection is disproportionately high, especially in immigrants from high burden TB countries. Policy makers have suggested a number of programs focusing on immigrant populations as part of a multi-level approach to identify latent disease, active disease, and reaching both documented and undocumented immigrant populations. NTM, however, represents an emerging public health problem with death rates on the sharp incline in the US. Given that NTM tends to be associated with older populations with comorbid conditions commonly associated with aging, the current population structure in the US will likely continue to explain future increases in NTM incidence. The cost of care for both diseases is likely to reflect these challenges. 
According to this study, the cost of care of managing mycobacterial diseases in the US between the years 2001 and 20012 was around $\$ 3$ billion. During the study period, the aggregated hospital cost increased for both diseases. For NTM, the increase in cost was at rate that exceeded healthcare inflation. For TB, this increase was at a lower rate than inflation, which arguably represents a decline. Also identified in this study were the unique demographic features of disease. For example, the strong correlation between NTM and the elderly was observed, which is concerning given the aging population structure of the US. As the large number of baby boomers enter their final years, the incidence of NTM is likely to demonstrate amplified increases, which could have a profound impact on healthcare costs.

For policy makers, this study has several important implications. First and foremost, NTM has been established as an emerging public health problem for which little is known. The epidemiology of NTM has not been widely measured on a national or global scale. The few data that exist are limited to select countries and care settings. Even piecing together currently available knowledge does not begin to capture the actual distribution of NTM disease on a national or global scale. As a result, the scope of the problem is not understood. Further, many scientific aspects of the disease remain unknown. Current literature has described various aspects of disease. First, risk groups have been well described. Also, the presence of infectious species in environmental 
samples, water, and the food have been documented. Further, NTM has found to be transmitted person to person in certain situations. Despite ongoing research, little is known about how transmission occurs. Therefore, the policy implications for NTM as a public health challenge represent a different type of approach traditionally undertaken by public health. With new diseases come new challenges that rival in importance to previous and successful public health policy. The ability of public health to recognize emerging problems that may not resemble those of centuries past represents a true test of policy makers in their ability to recognize and address public health challenges in all shapes and sizes. 


\section{REFERENCES}

${ }^{1}$ Madison, BM. Application of stains in clinical microbiology Biotech Histochem. 2001 May;76(3):119-25.

${ }^{2}$ Lawn, SD and Zumla, Al. Tuberculosis. Lancet. 2011 July 2;378(9785):57-72.

${ }^{3}$ Guzman JD, Gupta A, Bucar F, Gibbons S, Bhakta S. Antimycobacterials rom natural sources: ancient times, antibiotic era and novel scaffolds. Front Biosci (Landmark Ed). 2012 Jan 1;17:1861-1881.

${ }^{4}$ Lawn, SD and Zumla, Al. Tuberculosis. Lancet. 2011 July 2;378(9785):57-72.

${ }^{5}$ WL Salo, AC Aufderheide, J Buikstra, TA Holcomb. Identification of Mycobacterium tuberculosis DNA in a pre-Columbian Peruvian mummy. Proc Natl Acad Sci USA, 1994 Mar 15;91(6):1091-1094

${ }^{6}$ Nerlich AG, Haas CJ, Zink A, Szeimies U, Hagedorm HG. Molecular evidence for tuberculosis in an ancient Egyptian mummy. Lancet. 1997 Nov 8; 350(9088):1404.

${ }^{7}$ Rothschild BM, Martin LD, Lev G, et al. Mycobacterium tuberculosis Complex DNA from an Extinct Bison Dated 17,000 Years before the Present. Clin Infect Dis. 2001 Aug 1;33(3):305-311.

${ }^{8}$ Baker O, Lee OY, Wu HH, Besra GS, Minnikin DE, Llewellyn G, Williams $\mathrm{CM}$, Maixner F, O'Sullivan N, Zink A, Chamel B, Khawam R, Coqueugniot E, Helmer D, Le Mort F, Perrin P, Gourichon L, Dutailly B, Pálfi G, Coqueugniot $\mathrm{H}$, Dutour $\mathrm{O}$. Human tuberculosis predates domestication in ancient Syria. Tuberculosis (Edinb). 2015 Jun;95 Suppl 1:S4-S12

${ }^{9}$ Pósa A, Maixner F, Sola C, Bereczki Z, Molnár E, Masson M, Lovász G, Spekker O, Wicker E, Perrin P, Dutour O, Zink A, Pálfi G. Tuberculosis infection in a late-medieval Hungarian population. Tuberculosis (Edinb). 2015 Jun;95 Suppl 1:S60-4. 
${ }^{10}$ Pósa A, Maixner F, Mende BG, Köhler K, Osztás A, Sola C, Dutour O, Masson M, Molnár E, Pálfi G, Zink A. Tuberculosis in Late Neolithic-Early Copper Age human skeletal remains from Hungary. Tuberculosis (Edinb). 2015 Jun;95 Suppl 1:S18-22.

${ }^{11}$ Zink AR, Grabner W, Reischl U, Wolf H, Nerlich AG. Molecular study on human tuberculosis in three geographically distinct and time delineated populations from ancient Egypt. Epidemiol Infect. 2003 Apr;130(2):239249.

${ }^{12}$ Frith J. History of Tuberculosis. Part 1 - Phthisis, consumption and the White Plague. J Mil Vet Health. 2014 Jun;22(2):29-35.

${ }^{13}$ Lawn, SD and Zumla, Al. Tuberculosis. Lancet. 2011 July 2;378(9785):57-72.

${ }^{14}$ Frith J. History of Tuberculosis. Part 1 - Phthisis, consumption and the White Plague. J Mil Vet Health. 2014 Jun;22(2):29-35.

${ }^{15}$ Lawn, SD and Zumla, Al. Tuberculosis. Lancet. 2011 July 2;378(9785):57-72.

${ }^{16}$ Palfi G, Dutour O, Perrin P, Sola C and Zink A. Tuberculosis in evolution. Tuberculosis (Edinb). 2015 Jun;95 Suppl 1:S1-3.

${ }^{17}$ Palfi G, Dutour O, Perrin P, Sola C and Zink A. Tuberculosis in evolution. Tuberculosis (Edinb). 2015 Jun;95 Suppl 1:S1-3.

${ }^{18}$ Keshavjee $S$ and Farmer $P$. Tuberculosis, drug, resistance, and the history of modern medicine. N Engl J Med. 2012 Sep 6;367(10):931-936.

${ }^{19}$ Fogel N. Tuberculosis: A disease without boundaries. Tuberculosis (Edinb). 2015 Sep;95(5):527-531.

${ }^{20}$ Lee SS, Meintjes G, Kamarulzaman A, Leung CC. Management of tuberculosis and latent tuberculosis infection in human immunodeficiency virus-infected persons. Respirology. 2013 Aug;18(6):912—922.

${ }^{21}$ Lawn, SD and Zumla, Al. Tuberculosis. Lancet. $2011 \mathrm{Jul}$ 2;378(9785):57-72.

${ }^{22}$ Lee JY. Diagnosis and treatment of extrapulmonary tuberculosis. Tuberc Respir Dis (Seoul). 2015 Apr;78(2):47-55.

${ }^{23}$ Golden MP, Vikram HR. Extrapulmonary tuberculosis: an overview. Am Fam Physician. 2005 Nov 1;72(9):1761-1768. 
${ }^{24}$ Fogel N. Tuberculosis: A disease without boundaries. Tuberculosis. 2015 Sep:95(5);527-531.

${ }^{25}$ Restrepo, BI. Convergence of the tuberculosis and diabetes epidemics: renewal of old acquaintances. Clin In Dis. 2007 Aug 15;45(4):436-448.

${ }^{26}$ WHO website. Tuberculosis Fact sheet $N^{\circ} 104$. World Health Organization. October 2015. Available at: http://www.who.int/mediacentre/factsheets/fs104/en/. Accessed December $1,2015$.

${ }^{27}$ Centers for Disease Control and Prevention, website. TB Risk Factors. March 18, 2016. Available at: http://www.cdc.gov/tb/topic/basics/risk.htm Accessed July 13, 2016.

${ }^{28}$ Asner SA Morré SA, Bochud PY, Greub G. Host factors and genetic susceptibility to infections due to intracellular bacteria and fastidious organisms. Clin Microbiol Infect. 2014 Dec;20(12):1246-1253.

${ }^{29}$ Prabowo SA, Gröschel MI, Schmidt ED, Skrahina A, Mihaescu T, Hastürk S, Mitrofanov R, Pimkina E, Visontai I, de Jong B, Stanford JL, Cardona PJ, Kaufmann SH, van der Werf TS.Med Microbiol Immunol. Targeting multidrug-resistant tuberculosis (MDR-TB) by therapeutic vaccines. 2013 Apr;202(2):95-104.

${ }^{32}$ World Health Organization (WHO) website. Tuberculosis Fact sheet No104. World Health Organization. October 2015. Available at: http://www.who.int/mediacentre/factsheets/fs104/en/. Accessed December 1, 2015.

${ }^{33}$ Atkins PJ. Milk consumption and tuberculosis in Britain, 1850-1950. In Fenton A, ed. Order and Disorder: The Health Implications of Eating and Drinking In the Nineteenth and Twentieth Centuries. East Linton: Tuckwell Press, 2000: 83-95

${ }^{34}$ Atkins PJ. The pasteurization of milk in England: the science, culture and health implications of milk processing, 1900-1950. In: Smith D, Phillips J, eds. Food, Science, Policy and Regulation In The 20th Century. London: Routledge, 2000: 37-51

${ }^{35}$ Davies PD. Tuberculosis in humans and animals: are we a threat to each other? J R Soc Med. 2006 Oct; 99(10): 539-540.

${ }^{36}$ Lawn, SD and Zumla, Al. Tuberculosis. Lancet. 2011 July 2;378(9785):57-72.

${ }^{37}$ Szaluś-Jordanow O, Augustynowicz-Kopeć E, Czopowicz M, Olkowski 
A, Łobaczewski A, Rzewuska M, Sapierzyński R, Wiatr E, Garncarz M and Frymus T. Intracardiac tuberculomas caused by Mycobacterium tuberculosis in a dog. BMC Vet Res. 2016 Jun 14.12(1):109.

${ }^{38}$ Posthaus H, Bodmer T, Alves L, Oevermann A, Schiller I, Rhodes SG, Zimmerli S. Accidental infection of veterinary personnel with Mycobacterium tuberculosis at necropsy: A case study. Vet Microbiol. 2011 May 5;149(3-4):374-380.

${ }^{39}$ Ribeiro MG, Lima MC, Franco MM, Megid J, Soares LM, Machado LH, Miyata M, Pavan FR, Heinemann MB, Souza Filho AF, Lara GH, Sanches OC, Sanchez CD, Listoni FJ, Paes AC. Pre-Multidrug-Resistant Mycobacterium tuberculosis Infection Causing Fatal Enteric Disease in a Dog from a Family with History of Human Tuberculosis. Transbound Emerg Dis. 2016 Jun 30.

${ }^{40}$ Martinho AP, Franco MM, Ribeiro MG, Perrotti IB, Mangia SH, Megid J, Vulcano LC, Lara GH, Santos AC, Leite CQ, De Carvalho SO, Paes AC. Disseminated Mycobacterium tuberculosis infection in a dog. Am J Trop Med Hyg. 2013 Mar;88(3):596-600.

${ }^{41}$ Pérez-Lago L, Navarro Y, García-de-Viedma D. Current knowledge and pending challenges in zoonosis caused by Mycobacterium bovis: a review. Res Vet Sci. 2014 Oct;97 Suppl:S94-S100.

${ }^{42}$ Velayati AA, Farnia P, Mozafari M, MAlekshahlan D, Farahbod AM, Seif $S$, Rahideh A, Mirsaeidi M. Identification and Genotyping of Mycobacterium tuberculosis isolated from Water and Soil Samples of a Metropolitan City. Chest. 2015 Apr;147(4):1094-1102.

${ }^{43}$ Breuninger M, van GB, Philipsen RH, Mhimbira F, Hella JJ, Lwilla F, van den Hombergh J, Ross A, Jugheli L, Wagner D, Reither K. Diagnostic accuracy of computer-aided detection of pulmonary tuberculosis in chest radiographs: a validation study from sub-Saharan Africa. PLoS One. 2014 Sep 5;9(9):e106381.

${ }^{44}$ Golden MP, Vikram HR. Extrapulmonary tuberculosis: an overview. Am Fam Physician. 2005 Nov 1;72(9):1761-1768.

${ }^{45}$ Desikan P. Sputum smear microscopy in tuberculosis: Is it still relevant? Indian J Med Res. 2013 Mar;137(3):442-444.

${ }^{46}$ Molicotti P, Bua A, and Zanetti S. Cost-effectiveness in the diagnosis of tuberculosis: choices in developing countries. J Infect Dev Ctries. 2014 Jan 15:8(1):24-38.

${ }^{47}$ Desikan P. Sputum smear microscopy in tuberculosis: Is it still relevant? 
Indian J Med Res. 2013 Mar;137(3):442-444.

${ }^{48}$ Savini V, Fazii P, Favaro M, Astolfi D, Polilli E, Pompilio A, Vannucci M, D'Amario C, Di Bonaventura G, Fontana C, D'Antonio D.Tuberculosis-like pneumonias by the aerobic actinomycetes. Microbes Infect. 2012 May;14(5):401-410.

${ }^{49}$ Brisson-Noel A., Gicquel B., Lecossier D., Levy-Frebault V., Nassif X., Hance A. J. (1992) Rapid diagnosis of tuberculosis by amplification of mycobacterial DNA in clinical samples. Lancet 2:1069-1071

${ }^{50}$ Butler WR and Guthertz LS. Mycolic Acid Analysis by HighPerformance Liquid Chromatography for Identification of Mycobacterium Species. Clin Microbiol Rev. 2001 Oct;14(4):704-726

${ }^{51}$ Good R. New culture identification procedure initiated in CDC Mycobacteriology Lab. TB notes, Summer. Atlanta, GA: Division of TB Control, Centers for Disease Control;1990:13.

${ }^{52}$ Butler WR and Guthertz LS. Mycolic Acid Analysis by HighPerformance Liquid Chromatography for Identification of Mycobacterium Species. Clin Microbiol Rev. 2001 Oct;14(4):704-726

${ }^{53}$ UNITAID website. Tuberculosis: Diagnosing Technology and Market Landscape. 2015. Available at www.unitaid.org. Accessed April 21, 2016.

${ }^{54}$ Steigart K, Schiller I, Kloda LA, Boehme CC, Pai M, Dendukuri N. Xpert MTB/RIF assay for pulmonary tuberculosis and rifampin resistance in adults. Cochrane Database Sys Rev. 2013 Jan 31;(1):CD009593.

${ }^{55}$ Caminero JA, Sotgiu G, Zumla A, Migliori GB. Best drug treatment for multi-drug resistant and extensively drug resistant tuberculosis. Lancet Infect Dis. 2010 Sep;10(9):621-629.

${ }^{56}$ Palfi G, Dutour O, Perrin P, Sola $\mathrm{C}$ and Zink A. Tuberculosis in evolution. Tuberculosis (Edinb). 2015 June;95 Suppl 1:S1-3.

${ }^{57}$ Keshavjee $S$ and Farmer $P$. Tuberculosis, drug, resistance, and the history of modern medicine. N Engl J Med; 2012 Sep 6:367(10).931-936.

${ }^{58}$ Fogel N. Tuberculosis: A disease without boundaries. Tuberculosis. 2015 Sep:95(5);527-531.

59 UNITAID website. Tuberculosis: Diagnosing Technology and Market Landscape. 2015. Available at www.unitaid.org. Accessed April 21, 2016.

${ }^{60}$ Pantoja A, Kik SV, Denkinger CM. Costs of novel tuberculosis 
diagnostics: Will countries be able to afford it? J Infect Dis. 2015 Apr 1;211 Suppl 2:S67-S77.

${ }^{61}$ Blumberg HM, Burman WJ, Chaisson RE, et al. American Thoracic Society/Centers for Disease Control and Prevention/Infectious Diseases Society of America; treatment of tuberculosis. Am J Crit Care Med. 2003 Feb 15;167(4):603-662.

${ }^{62}$ Caminero JA, Sotgiu G, Zumla A, Migliori GB. Best drug treatment for multi-drug resistant and extensively drug resistant tuberculosis. Lancet Infect Dis. 2010 Sep;10(9):621-629.

${ }^{63}$ Areeshi MY, Bisht SC, Mandal RK, Haque S. Prevalence of drug resistance in clinical isolates of tuberculosis from GCC: a literature review from January 2002 to March 2013. J Infect Dev Ctries. 2014 Sep 12;8(9):1137-1147.

${ }^{64}$ Mishra R, Shukla P, Huang W, Hu N. Gene mutations in Mycobacterium tuberculosis: multidrug-resistant TB as an emerging global public health crisis. Tuberculosis (Edinb). 2015 Jan;95(1):1-5.

${ }^{65}$ Mishra R, Shukla P, Huang W, Hu N. Gene mutations in Mycobacterium tuberculosis: multidrug-resistant TB as an emerging global public health crisis. Tuberculosis (Edinb). 2015 Jan;95(1):1-5.

${ }^{66}$ Gupta A, Anupurba S. Detection of drug resistance in Mycobacterium tuberculosis: Methods, principles and applications. Indian J Tuberc. 2015 Jan;62(1):13-22.

${ }^{67}$ WHO website. Tuberculosis Fact sheet N ${ }^{\circ} 104$. World Health Organization. October 2015. Available at:

http://www.who.int/mediacentre/factsheets/fs104/en/. Accessed December 1, 2015.

${ }^{68}$ WHO website. Tuberculosis Fact sheet $N^{\circ} 104$. World Health Organization. October 2015. Available at: http://www.who.int/mediacentre/factsheets/fs104/en/. Accessed December $1,2015$.

${ }^{69}$ WHO website. Tuberculosis Fact sheet $N^{\circ} 104$. World Health Organization. October 2015. Available at: http://www.who.int/mediacentre/factsheets/fs104/en/. Accessed December 1, 2015.

${ }^{70}$ SD Lawn, G Churchyard. Epidemiology of HIV-associated tuberculosis. Curr Opin HIV AIDS. 2009 Jul;4(4):325-33. 
${ }^{71}$ Lawn, SD and Zumla, Al. Tuberculosis. Lancet. 2011 July 2;378(9785):57-72.

${ }^{72}$ Golden MP, Vikram HR. Extrapulmonary tuberculosis: an overview. Am Fam Physician. 2005 Nov 1;72(9):1761-1768.

${ }^{73}$ WHO website. Tuberculosis Fact sheet $N^{\circ} 104$. World Health Organization. October 2015. Available at:

http://www.who.int/mediacentre/factsheets/fs104/en/. Accessed December 1, 2015.

${ }^{74}$ Lawn, SD and Zumla, Al. Tuberculosis. Lancet. 2011 July 2;378(9785):57-72.

${ }^{75}$ WHO website. Tuberculosis Fact sheet $N^{\circ} 104$. World Health Organization. October 2015. Available at:

http://www.who.int/mediacentre/factsheets/fs104/en/. Accessed December $1,2015$.

${ }^{76}$ Brode SK, Daley CL and Marras TK. The epidemiologic relationship between tuberculosis and non-tuberculosis mycobacterial disease: a systematic review. Int J Tuberc Lung Dis. 2014 Nov;18(11):1370-1377.

${ }^{77}$ Adjemian J. Olivier KN, Seitz AE Holland SM and Prevots DR. Prevalence of Nontuberculous mycobacterial lung disease in U.S. medicare beneficiaries. Am J Respir Crit Care Med. 2012 Apr 15;185(8): 881-886.

${ }^{78}$ Billinger ME, O,ivier KN, Viboud $\mathrm{C}$ et al. Nontuberculous mycobacteriaassociated lung disease in hospitalized persons, United States, 19982005. Emerg Infect Dis. 2009 Oct;15(10):1562-1569.

${ }^{79}$ Mehta M and Marras TK. Impaired health-related quality of life in pulmonary Nontuberculous mycobacterial disease. Respir Med. 2011 Nov;105(11):1718-1725.

${ }^{80}$ Adjemian J. Olivier KN, Seitz AE Holland SM and Prevots DR. Prevalence of Nontuberculous mycobacterial lung disease in U.S. medicare beneficiaries. Am J Respir Crit Care Med. 2012 Apr 15;185(8): 881-886.

${ }^{81}$ Mirsaedi M, Farshidpour M, Allen MB, Ebrahami G, Falkinham JO. Highlight on Advances in Nontuberculous Mycobacterial Disease in North America. Biomed Res Int. 2014;2014:919474.

${ }^{82}$ Runyon EH. Anonymous mycobacteria in pulmonary disease. Med Clin 
North Am.1959 Jan;43(1):273-290.

${ }^{83}$ Rogall T, Wolters J, Flohr T, Böttger EC. Towards a phylogeny and definition of species at the molecular level within the genus Mycobacterium. 1990 Oct. J Clin Microbiol;40(4):323-330.

${ }^{84}$ Falkinham, JO III. Epidemiology of infection by nontuberculous mycobacteria. Clin Microbiol Rev.1996;9(2):177-215.

${ }^{85}$ Tortoli E. Microbiological Features and clinical relevance of new species of the genus Mycobacterium. Clin Microbiol Rev. 2014 Oct;27(4):727-752.

${ }^{86}$ Management of opportunist mycobacterial infections: Joint Tuberculosis Committee Guidelines 1999. Subcommittee of the Joint Tuberculosis Committee of the British Thoracic Society. Thorax. 2000 Mar; 55(3):210218.

${ }^{87}$ Diagnosis and treatment of disease caused by nontuberculous mycobacteria. This official statement of the American Thoracic Society was approved by the Board of Directors, March 1997. Medical Section of the American Lung Association. Am J Respir Crit Care Med. 1997 Aug; 156(2 Pt 2):S1-25.

${ }^{88}$ Raju RM, Raju SM, Zhao Y and Rubin EJ. Leveraging Advances in Tuberculosis Diagnosis and Treatment to Address Nontuberculous Mycobacterial Disease. Emerg Infect Dis. 2016 Mar;22(3): 365-369

${ }^{89}$ Kasperbauer S and Huitt G. Management of extrapulmonary nontuberculous mycobacterial infections. Semin Respir Crit Care Med. 2013 Feb;34(1):143-150.

${ }^{90}$ Parker NP, Scott AR, Finkelstein M, Tibesar RJ, Lander TA, Rimell FL, Sidman JD. Predicting surgical outcomes in pediatric cervicofacial nontuberculous mycobacterial lymphadenitis. Ann Otol Rhinol Laryngol. 2012 Jul;121(7):478-484.

${ }^{91}$ Laquer $\mathrm{V}, \mathrm{Ta} \mathrm{T}$, Nguyen T, Tan B. Mycobacterium poriferae infection in a psoriasis patient on anti-TNF- $\alpha$ therapy. Dermatol Online J. 2013 Sep;19(9):19609

${ }^{92}$ Lembo G, Goldstein EJ, Troum O, Mandelbaum B. Successful treatment of mycobacterium terrae complex infection of the knee. J Clin Rheumatol. 2012 Oct;18(7):359-362.

${ }^{93}$ Prevots DR, Shaw PA, Strickland D. et al. Nontuberculous mycobacterial lung disease prevalence at four integrated care delivery systems. Am J Respir Crit Care Med. 2010 Oct 1;182(7):970-976. 
${ }^{94} \mathrm{Kim} \mathrm{CJ}$, Kim NH, Song KH, et al. Differentiating rapid-and-slow growing mycobacteria by difference in time to growth deletion in liquid media. Diagn Microbiol Infect Dis. 2013 Jan;75(1):73-76.

${ }^{95}$ Machado D, Ramos J, Couto I et al. Assessment of the BD MGIT TBC identification test for the detection of Mycobacterium tuberculosis complex in a network of mycobacteriology laboratories. Biomed Res Int. 2014; 2014: 398108.

${ }^{96}$ Cayrou C, Turenne C, Behr MA and Drancourt M. Genotyping of Mycobacterium avium complex organisms using multi-spacer sequence typing. Microbiology. 2010 Mar;156(Pt 3):687-694.

${ }^{97}$ Shojaei H, Heidarieh P, Hashemi A, Feizabadi MM, Daei Naser A. Species identification of neglected nontuberculous mycobacteria in a developing country. Jpn J Infect Dis. 2011;64(4):265-271.

${ }^{98}$ Hoefsloot W, van Ingen J, Andrejak C, et al. The geographic diversity of nontuberculous mycobacteria isolated from pulmonary samples: an NTMNET collaborative study. Eur Respir J. 2013 Dec:42(6);1604-1613.

${ }^{99}$ Griffith DE, Aksamit T, Brown-Elliott BA, Catanzaro A, Daley C, Gordin F, Holland SM, Horsburgh R, Huitt G, lademarco MF, Iseman M, Olivier K, Ruoss S, von Reyn CF, Wallace RJ Jr, Winthrop K; ATS Mycobacterial Diseases Subcommittee; American Thoracic Society; Infectious Disease Society of America. An official ATS/IDSA statement: diagnosis, treatment, and prevention of nontuberculous mycobacterial diseases. Am J Respir Crit Care Med. 2007 Feb 15;175(4):367-416.

${ }^{100}$ Mirsaeidi M. Personalized medicine approach in mycobacterial disease. Int J Mycobacteriol. 2012 Jun;1(2):59-64.

101 Dega H, Robert J, Bonnafous P, Jarlier V, Grosset J. Activities of several antimicrobials against Mycobacterium ulcerans infection in mice. Antimicrobial Agents and Chemotherapy. 2000;44(9):2367-2372.

${ }^{102}$ Mirsaeidi M. Personalized medicine approach in mycobacterial disease. Int J Mycobacteriol. 2012 Jun;1(2):59-64.

${ }^{103}$ Mirsaeidi M, Farshidpour M, Ebrahimi G, Aliberti S, Falkinham JO, III Management of nontuberculous mycobacterial infection in the elderly. Eur J Int Med. 2014;25(4):356-363.

${ }^{104}$ Fukuchi $Y$. The aging lung and chronic obstructive pulmonary disease: similarity and difference. Proc Am Thorac Soc. 2009 Dec 1; 6(7):570-572.

${ }^{105}$ Keating MR and Daly JS. Nontuberculous mycobacterial infections in 
solid organ transplantation. American Journal of Transplantation. 2013;13(supplement 4):77-82.

${ }^{106}$ Winthrop K. L., Chang E., Yamashita S., lademarco M. F., LoBue P. A. Nontuberculous mycobacteria infections and anti-tumor necrosis factor- $\alpha$ therapy. Emerg Infect Dis. 2009;15(10):1556-1561.

${ }^{107}$ Chan ED and Iseman MD. Underlying host risk factors for nontuberculous mycobacterial lung disease. Semin in Respir Crit Care Med. 2013 Feb;34(1):110-123.

${ }^{108}$ Leung JM and Olivier IN. Nontuberculous mycobacteria: The changing epidemiology and treatment challenges in cystic fibrosis. Curr Opin Pulm Med. 2013 Nov;19(6):662-669.

${ }^{109}$ Dorman S and Subramanian A. Nontuberculous mycobacteria in solid organ transplant recipients. Am J Transplant. 2009;9,Suppl 4:S63-S69.

110 Piersimoni C. Nontuberculous mycobacteria infection in solid organ transplant recipients. Eur J Clin Microbiol Infect Dis. 2012;31(4):397-403.

${ }^{111}$ Knoll BM, Kappagoda S, Gill RR, Goldberg HJ, Boyle K, Baden LR, Fuhlbrigge AL, Marty FM. Non-tuberculous mycobacterial infection among lung transplant recipients: a 15-year cohort study. Transpl Infecti Dis. 2012 Oct;14(5):452-460.

${ }^{112}$ Adjemian J, Olivier KN, Seitz AE, Holland SM, Prevots DR. Prevalence of nontuberculous mycobacterial lung disease in U.S. Medicare beneficiaries. Am J Respir Crit Care Med. 2012 Apr 15; 185(8):881-6.

${ }^{113}$ Mirsaedi M, Farshidpour M, Allen MB, Ebrahami G, Falkinham JO. Highlight on Advances in Nontuberculous Mycobacterial Disease in North America. BioMed Res Int. 2014;2-14:919474.

114 Mirsaeidi M, Hadid W, Ericsoussi B, Rodgers D, Sadikot RT. Nontuberculous mycobacterial disease is common in patients with non-cystic fibrosis bronchiectasis. Int J Infect Dis. 2013 Nov;17(11):e1000-e1004.

${ }^{115}$ Kartalija M, Ovrutsky AR, Bryan CL, Pott GB, Fantuzzi G, Thomas J, Strand MJ, Bai X, Ramamoorthy P, Rothman MS, Nagabhushanam V., McDermott M, Levin AR, Frazer-Abel A, Giclas PC, Korner J, Iseman MD, Shapiro L, Chan ED. Patients with nontuberculous mycobacterial lung disease exhibit unique body and immune phenotypes. Am J Respir Crit Care Med. 2013 Jan;187(2):197-205.

${ }^{116}$ Chan ED and Iseman MD. Slender, older women appear to be more susceptible to nontuberculous mycobacterial lung disease. Gend Med. 
2010 Feb;7(1):5-18.

${ }^{117}$ Mirsaeidi M, Farshidpour M, Ebrahimi G, Aliberti S, Falkinham JO, 3rd. Management of nontuberculous mycobacterial infection in the elderly. Eur J Intern Med. 2014 Apr;25(4):356-363.

${ }^{118}$ Fowler SJ, French J, Screaton NJ, Foweraker J, Condliffe A, Haworth CS, Exley AR, Bilton D. Nontuberculous mycobacteria in bronchiectasis: Prevalence and patient characteristics. Eur Respir J. 2006;28(6):12041210.

119 Ito A, Hashimoto T, Ishida T, Tachibana H, Korogi Y, Tokioka F, Yoshioka $\mathrm{H}$. A case of lung disease and spondylitis due to Mycobacterium intracellulare in a immunocompetent patient. Kekkaku. 2013 Jun;88(6):559-564.

120 Khatter S, Singh UB, Arora J, Rana T, Seth P. Mycobacterial infections in human immuno-deficiency virus seropositive patients: role of non-tuberculous mycobacteria. Indian J Tuberc. 2008 Jan;55(1):28-33.

${ }^{121}$ Arastéh KN, Cordes C, Ewers M, Simon V, Dietz E, Futh UM, Brockmeyer NH, L'age MP. HIV-related nontuberculous mycobacterial infection: incidence, survival analysis and associated risk factors. Eur J Med Res. 2000 Oct 30;5(10):424-430.

${ }^{123}$ Grabar S, Weiss L and Costagliola D. HIV infection in older patients in the HAART era. J Antimicrob Chemother 2006 Jan;57(1):4-7.

${ }^{124}$ Centers for Disease Control and Prevent (CDC) website. HIV in persons 50 and over. April 4, 2016. Accessed July 21, 2016.

${ }^{125}$ Altschuler J, Katz AD, Tynan M. Developing and implementing an HIVIAIDS educational curriculum for older adults. Gerontologist. 2004 Feb;44(1):121-126.

${ }^{126}$ Centers for Disease Control and Prevent (CDC) website. HIV in persons 50 and over. April 4, 2016. Accessed July 21, 2016.

${ }^{127}$ Karlovsky M, Lebed B, Mydlo JH. Increasing incidence and importance of HIVIAIDS and gonorrhea among men aged $>/=50$ years in the US in the era of erectile dysfunction therapy. Scand J Urol Nephrol. 2004;38(3):247-252.

${ }^{128}$ Centers for Disease Control and Prevent (CDC) website. HIV in persons 50 and over. April 4, 2016. Accessed July 21, 2016.

${ }^{129}$ Zelenetz PD, Epstein ME. HIV in the elderly. AIDS Patient Care STDS. 
1998 Apr;12(4):255-262.

${ }^{130}$ Mirsaeidi M. Nontuberculous Mycobacterial Disease Mortality in the United States, 1999-2010: A Population-Based Comparative Study. PLOS ONE. 2014 Mar 14;9(3):91879.

131 Halstrom S, Price P, Thomson R. Review: Environmental mycobacteria as a cause of human infection. Int J Mycobacteriol. 2015 Jun;4(2):81-91.

132 Velayati AA, Rahideh S, Nezhad ZD, Farnia P and Mirsaeidi M. Nontuberculous mycobacteria in the Middle East: Current situation and future challenges. Int J Mycobacteriol. 2015 Mar;4(1):7-17.

133 livanainen EK, Martikainen PJ, Räisänen ML, and Katila ML. Mycobacteria in boreal coniferous forest soils. FEMS Microbiol. Ecol. 1997; 23: 325-332.

${ }^{134}$ Ichiyama S, Shimokata K, and Tsukamura M. The isolation of Mycobacterium avium complex from soil, water, and dusts. Microbiol. Immunol. 1988;32(7):733-739.

135 livanainen $E$, Martikainen $P$, Väänänen $P$, and Katila $M L$. Environmental factors affecting the occurrence of mycobacteria in brook waters. Appl Environ Microbiol. 1993 Feb;59(2):398-404.

${ }^{136}$ Whiley H, Keegan A, Fallowfield H, Bentham R. The presence of opportunistic pathogens, Legionella spp., L. pneumophila and Mycobacterium avium complex, in South Australian reuse water distribution pipelines. J Water Health. 2015 Jun;13(2):553-561.

${ }^{137}$ Nishiuchi Y, Maekura R, Kitada S, Tamaru A, Taguri T, Kira Y, Hiraga T, Hirotani A, Yoshimura K, Miki M, Ito M. The recovery of Mycobacterium avium-intracellulare complex (MAC) from the residential bathrooms of patients with pulmonary MAC. Clin Infect Dis. 2007 Aug 1;45(3):347-351.

138 Thomson, Rachel. (2013) Characteristics of Nontuberculous Mycobacteria from a Municipal Water Distribution System and their Relevance to Human Infections (Doctoral dissertation). Retrieved from QUT ePrints. (ID Code 65483).

${ }^{139}$ Falkinham JO. Nontuberculous mycobacteria from household plumbing of patients with nontuberculous mycobacteria disease. Emerg Infect Dis. 2011 Mar; 17(3):419-424.

${ }^{140}$ Wali SO, Abdelaziz MM, Krayem AB, Samman YS, Shukairi AN, Mirdad SA, Albanna AS, Alghamdi HJ, Osoba AO. The presence of 
atypical mycobacteria in the mouthwashes of normal subjects: role of tap water and oral hygiene. Ann Thorac Med. 2008 Jan;3(1):5-8.

${ }^{141}$ Ashbolt NJ. Environmental (Saprozoic) Pathogens of Engineered Water Systems: Understanding Their Ecology for Risk Assessment and Management. Pathogens. 2015 Jun 19;4(2):390-405.

142 Thomson R, Tolson C, Carter R, Coulter C, Huygens F, Hargreaves $M$. Isolation of nontuberculous mycobacteria (NTM) from household water and shower aerosols in patients with pulmonary disease caused by NTM. Microbiol. 2013 Sep;51(9):3006-3011.

${ }^{143}$ Glazer CS, Martyny JW, Lee B, Sanchez TL, Sells TM, Newman LS, Murphy J, Heifets L, Rose CS. Nontuberculous mycobacteria in aerosol droplets and bulk water samples from therapy pools and hot tubs. J Occup Environ Hyg. 2007 Nov; 4(11):831-840.

${ }^{144}$ Lorencova A, Klanicova B, Makovcova J, Slana I, Vojkovska H, Babak $V$, Pavlik I, Slany M. Nontuberculous mycobacteria in freshwater fish and fish products intended for human consumption. Foodborne Pathog Dis. 2013 Jun;10(6):573-576.

${ }^{145}$ Bryant, JM, Grogono DM, Greaves D, Foweraker J, Roddick I, Inns T, Reacher M, Haworth CS, Curran MD, Harris SR, Peacock SJ, Parkhill J, Floto RA. Whole-genome sequencing to identify transmission of Mycobacterium abscessus between patients with cystic fibrosis: a retrospective cohort study. Lancet, 2013 May 4; 381(9877):1551-1560.

146 Halstrom S, Price P, Thomson R. Review: Environmental mycobacteria as a cause of human infection. Int J Mycobacteriol. 2015 Jun;4(2):81-91.

${ }^{147}$ van Ingen J. Diagnosis of nontuberculous mycobacterial infections. Seminars in Respiratory and Critical Care Medicine. 2013 Feb;34(1):103109.

${ }^{148}$ de Bel A, de Geyter D, de Schutter I, Mouton C, Wellemans I, Hanssens L, Schelstraete P, Malfroot A, Pierard D. Sampling and decontamination method for culture of Nontuberculous Mycobacteria in respiratory samples of cystic fibrosis patients. J Clin Microbiol. 2013 Dec;51(12):4204-4206.

149 Saleeb P, Olivier KN. Pulmonary nontuberculous mycobacterial disease: new insights into risk factors for susceptibility, epidemiology, and approaches to management in immunocompetent and immunocompromised patients. Curr Infect Dis Rep. 2010 May;12(3):198203. 
150 Jagielski T, Van Ingen J, Rastogi N, Dziadek J, Mazur PK, Bielecki J. Current methods in the molecular typing of Mycobacterium tuberculosis and other Mycobacteria. BioMed Res Int. 2014;2014:645802.

151 Jeong J, Kim SR, Lee SH, Lim JH, Choi JI, Park JS, Chang CL, Choi JY, Richman DD, Smith DM. The use of high performance liquid chromatography to speciate and characterize the epidemiology of mycobacteria. Lab Med. 2011 Oct;42(10):612-617.

${ }^{152}$ Butler WR and Guthertz LS. Mycolic Acid Analysis by HighPerformance Liquid Chromatography for Identification of Mycobacterium Species. Clin Microbiol Rev. 2001 Oct;14(4):704-726

${ }^{153}$ Rogers JT, Procop GW, Steelman CK, Abramowsky CR, Tuohy MT, Shehata BM. Clinical utility of DNA amplification and sequencing to identify a strain of Mycobacterium avium in paraffin-embedded, formalinfixed biopsies from an immunosuppressed child. Pediatr Dev Pathol. 2012 Jul-Aug;15(4):315-317.

${ }^{154}$ Butler WR and Guthertz LS. Mycolic Acid Analysis by HighPerformance Liquid Chromatography for Identification of Mycobacterium Species. Clin Microbiol Rev. 2001 Oct;14(4):704-726

155 Varma-Basil M, Garima K, Pathak R, Dwivedi SKD, Narang A, Bhatnagar A, Bose M. Development of a novel pcr restriction analysis of the hsp65 gene as a rapid method to screen for the Mycobacterium tuberculosis complex and nontuberculous mycobacteria in high-burden countries. J Clin Microbiol. 2013 Apr;51(4):1165-1170.

156 Jang MA, Koh WJ, Huh HI, Kim SY, Jeon K,, Ki CS, Lee NY. Distribution of nontuberculous mycobacteria by multigene sequencebased typing and clinical significance of isolated strains. J Clin Microbiol. 2014 Apr;52(4):1207-1212.

${ }^{157}$ Park JS, Choi JI, Lim JH, Ahn JJ, Jegal Y, Seo KW, Ra SW, Jeon JB, Lee SH, Kim SR, Jeong J. The combination of real-time PCR and HPLC for the identification of non-tuberculous mycobacteria. Ann Lab Med. 2013 Sep;33(5):349-352.

158 Griffith DE, Aksamit T, Brown-Elliott BA, Catanzaro A, Daley C, Gordin F, Holland SM, Horsburgh R, Huitt G, lademarco MF, Iseman M, Olivier K, Ruoss S, von Reyn CF, Wallace RJ Jr, Winthrop K; ATS Mycobacterial Diseases Subcommittee; American Thoracic Society; Infectious Disease Society of America. An official ATS/IDSA statement: diagnosis, treatment, and prevention of nontuberculous mycobacterial diseases. Am J Respir Crit Care Med. 2007 Feb 15;175(4):367-416. 
159 Wang HC, Liaw YS, Yang PC, Kuo SH, Luh KT. A pseudoepidemic of Mycobacterium chelonae infection caused by contamination of a fibreoptic bronchoscope suction channel. Eur Respir J. 1995 Aug;8(8):1259-1262.

${ }^{160}$ Savini V, Fazii P, Favaro M, Astolfi D, Polilli E, Pompilio A, Vannucci M, D'Amario C, Di Bonaventura G, Fontana C, D'Antonio D.Tuberculosis-like pneumonias by the aerobic actinomycetes. Microbes Infect. 2012 May;14(5):401-410.

${ }^{161}$ Griffith DE, Aksamit T, Brown-Elliott BA, Catanzaro A, Daley C, Gordin F, Holland SM, Horsburgh R, Huitt G, lademarco MF, Iseman M, Olivier K, Ruoss S, von Reyn CF, Wallace RJ Jr, Winthrop K; ATS Mycobacterial Diseases Subcommittee; American Thoracic Society; Infectious Disease Society of America. An official ATS/IDSA statement: diagnosis, treatment, and prevention of nontuberculous mycobacterial diseases. Am J Respir Crit Care Med. 2007 Feb 15;175(4):367-416.

162 Arend SM, Van Soolingen D, Ottenhoff TH. Diagnosis and treatment of lung infection with nontuberculous mycobacteria. Curr Opin Pulm Med. 2009 May;15(3):201-208.

${ }^{164}$ Griffith DE, Aksamit T, Brown-Elliott BA, Catanzaro A, Daley C, Gordin F, Holland SM, Horsburgh R, Huitt G, lademarco MF, Iseman M, Olivier K, Ruoss S, von Reyn CF, Wallace RJ Jr, Winthrop K; ATS Mycobacterial Diseases Subcommittee; American Thoracic Society; Infectious Disease Society of America. An official ATS/IDSA statement: diagnosis, treatment, and prevention of nontuberculous mycobacterial diseases. Am J Respir Crit Care Med. 2007 Feb 15;175(4):367-416.

165 Griffith DE, Aksamit T, Brown-Elliott BA, Catanzaro A, Daley C, Gordin F, Holland SM, Horsburgh R, Huitt G, lademarco MF, Iseman M, Olivier K, Ruoss S, von Reyn CF, Wallace RJ Jr, Winthrop K; ATS Mycobacterial Diseases Subcommittee; American Thoracic Society; Infectious Disease Society of America. An official ATS/IDSA statement: diagnosis, treatment, and prevention of nontuberculous mycobacterial diseases. Am J Respir Crit Care Med. 2007 Feb 15;175(4):367-416.

${ }^{167}$ Griffith DE, Aksamit T, Brown-Elliott BA, Catanzaro A, Daley C, Gordin F, Holland SM, Horsburgh R, Huitt G, lademarco MF, Iseman M, Olivier K, Ruoss S, von Reyn CF, Wallace RJ Jr, Winthrop K; ATS Mycobacterial Diseases Subcommittee; American Thoracic Society; Infectious Disease Society of America. An official ATS/IDSA statement: diagnosis, treatment, and prevention of nontuberculous mycobacterial diseases. Am J Respir Crit Care Med. 2007 Feb 15;175(4):367-416.

${ }^{168}$ Griffith DE, Aksamit T, Brown-Elliott BA, Catanzaro A, Daley C, Gordin 
F, Holland SM, Horsburgh R, Huitt G, lademarco MF, Iseman M, Olivier K, Ruoss S, von Reyn CF, Wallace RJ Jr, Winthrop K; ATS Mycobacterial Diseases Subcommittee; American Thoracic Society; Infectious Disease Society of America. An official ATS/IDSA statement: diagnosis, treatment, and prevention of nontuberculous mycobacterial diseases. Am J Respir Crit Care Med. 2007 Feb 15;175(4):367-416.

169 Griffith DE, Aksamit T, Brown-Elliott BA, Catanzaro A, Daley C, Gordin F, Holland SM, Horsburgh R, Huitt G, lademarco MF, Iseman M, Olivier K, Ruoss S, von Reyn CF, Wallace RJ Jr, Winthrop K; ATS Mycobacterial Diseases Subcommittee; American Thoracic Society; Infectious Disease Society of America. An official ATS/IDSA statement: diagnosis, treatment, and prevention of nontuberculous mycobacterial diseases. Am J Respir Crit Care Med. 2007 Feb 15;175(4):367-416.

${ }^{170}$ Westphal JF. Macrolide - induced clinically relevant drug interactions with cytochrome P-450A (CYP) 3A4: an update focused on clarithromycin, azithromycin and dirithromycin. Br J Clin Pharmacol. 2000 Oct; 50(4):285295.

${ }^{171}$ Griffith DE and Aksamit TR. Therapy of refractory nontuberculous mycobacterial lung disease. Curr Opin Infect Dis. 2012 Apr;25(2):218227.

172 Jarand J, Levin A, Zhang L, Huitt G, Mitchell JD, Daley CL. Clinical and microbiologic outcomes in patients receiving treatment for Mycobacterium abscessus pulmonary disease. Clin Infect Dis. 2011 Mar 1;52(5):565-571.

173 Jeon K, Kwon OJ, Nam YL, Kim BJ, Kook YH, Lee SH, Young KP, Chang KK, Koh WJ. Antibiotic treatment of Mycobacterium abscessus lung disease: a retrospective analysis of 65 patients. Am J Respir Crit Care Med. 2009 Nov 1;180(9):896-902.

${ }^{174}$ Margaret $\mathrm{M}$ and Johnson JAO. Nontuberculous mycobacterial pulmonary infections. J Thorac Dis. 2014 Mar;6(3):210-220.

${ }^{175}$ Cassidy PM, Hedberg K, Saulson A, McNelly E and Winthrop KL. Nontuberculous prevalence and risk factors: a changing epidemiology. Clin Infect Dis. 2009 Dec 15;49(12):124-129.

${ }^{176}$ Mirsaeidi M, Machado RF, Garcia JG, Schraufnagel DE. Nontuberculous mycobacterial disease mortality in the United States, 1999-2010: a population-based comparative study. PLoS ONE. 2014;9(3):e91879.

${ }^{177}$ Andréjak C, Thomsen VØ, Johansen IS, Riis A, Benfield TL, Duhaut P, Sørensen HT, Lescure FX, Thomsen RW. Nontuberculous pulmonary 
mycobacteriosis in Denmark: incidence and prognostic factors. Am J Respir Crit Care Med. 2010 Mar 1;181(5):514-521.

${ }^{178}$ Mirsaeidi M, Machado RF, Garcia JGN, Schraufnagel DE. Nontuberculous mycobacterial disease mortality in the United States, 1999-2010: a population-based comparative study. PLoS ONE. 2014;9(3):e91879.

${ }^{179}$ Yeh JJ, Wang YC, Lin CL, Chou CY, Yeh TC, Wu BT, Sung FC, Kao $\mathrm{CH}$. Nontuberculous mycobacterial infection is associated with increased respiratory failure: a nationwide cohort study. PLoS One. 2014 Jun 11;9(6):e99260.

180 Iseman MD and Marras TK. The importance of Nontuberculous mycobacterial lung disease. Am J Respir Crit Care Med. 2008 Nov 15;178(10):999-1000.

${ }^{181}$ Marras TK. Chedore P, Ying AM, Jamieson F. Isolation prevalence of pulmonary nontuberculous mycobacteria in Ontario, 1997-2003. Thorax. 2007 Aug.62(8);661-666.

${ }^{182}$ Winthrop KL, McNelley E, Kendall B, Marshall-Olson A, Morris C, Cassidy M, Saulson A, Hedberg K. Pulmonary nontuberculous mycobacterial disease prevalence and clinical features: an emerging public health disease. Am J Respir Crit Care Med. 2010 Oct 1;182(7):977982.

${ }^{183}$ Prevots DR, Shaw PA, Strickland D, Jackson LA, Raebel MA, Blosky MA, De Oca RM, Shea YR, Seitz AE, Holland SM, Olivier KN.

Nontuberculous mycobacterial lung disease prevalence at four integrated health care delivery systems. Am J Respir Crit Care Med. 2010 Oct 1;182(7):970-976.

${ }^{184}$ Adjemian J, Olivier KN, Seitz AE, Falkinham JO, III, Holland SM, Prevots DR. Spatial clusters of nontuberculous mycobacterial lung disease in the United States. Am J Respir Crit Care Med. 2012 Sep $15 ; 186(6): 553-558$.

${ }^{185}$ Margaret M, Johnson JAO. Nontuberculous mycobacterial pulmonary infections. Journal of Thoracic Disease. 2014 Mar;6(3):210-220.

${ }^{186}$ Wiener JM, Tilly J Population ageing in the United States of America: implications for public programmes. Int J Epidemiol. 2002 Aug;31(4):776781.

187 United States Census Bureau website. U.S. Census Bureau Projections Show a Slower Growing, Older, More Diverse Nation a Half 
Century from Now. December 12, 2012. Available at www.census.gov. Accessed August 24, 2016.

${ }^{188}$ Wiener JM, Tilly J Population ageing in the United States of America: implications for public programmes. Int J Epidemiol. 2002 Aug; 31(4):776781.

189 United States Census Bureau website. U.S. Census Bureau Projections Show a Slower Growing, Older, More Diverse Nation a Half Century from Now. December 12, 2012. Available at www.census.gov. Accessed August 24, 2016.

${ }^{191}$ Rynning E. The aging populations of Europe-implications for health systems and patients' rights. Eur J Health Law. 2008 Sep;15(3):297-306.

${ }^{193}$ Adjemian J, Olivier KN, Seitz AE, Falkinham JO, III, Holland SM, Prevots DR. Spatial clusters of nontuberculous mycobacterial lung disease in the United States. Am J Respir Crit Care Med. 2012 Sep 15;186(6):553-558.

${ }^{194}$ Akbar Velayati A, Farnia P, Mozafari M, Malekshahian D, Seif S, Rahideh S, Mirsaeidi M. Molecular epidemiology of nontuberculous mycobacteria isolates from clinical and environmental sources of a metropolitan city. PloS one. 2014 Dec 8;9(12):e114428.

${ }^{195}$ Masson AM, Prissick FH. Cervical lymphadenitis in children caused by chromogenic Mycobacteria. Can Med Assoc J. 1956 Nov 15;75(10):798803.

${ }^{196}$ Mirsaeidi M, Farshidpour M, Ebrahimi G, Aliberti S, Falkinham JO, 3rd. Management of nontuberculous mycobacterial infection in the elderly. Eur J Intern Med. 2014 Apr;25(4):356-363.

${ }^{197}$ Marras TK, Daley CL. Epidemiology of human pulmonary infection with nontuberculous mycobacteria. Clin Chest Med. 2002 Sep;23(3):553-567.

198 Akbar Velayati A, Farnia P, Mozafari M, Malekshahian D, Seif S, Rahideh S, Mirsaeidi M. Molecular epidemiology of nontuberculous mycobacteria isolates from clinical and environmental sources of a metropolitan city. PloS one. 2014 Dec 8;9(12):e114428.

${ }^{199}$ Khan K, Wang J, Marras TK. Nontuberculous mycobacterial sensitization in the United States: national trends over three decades. Am J Respir Crit Care Med. 2007 Aug 1;176(3):306-313. 
${ }^{200}$ Kendall BA, Varley CD, Choi D, et al. Distinguishing tuberculosis from nontuberculous mycobacteria lung disease, Oregon, USA. Emerging infectious diseases. 2011 Mar;17(3):506-509.

201 Mezochow A, Hamilton K, Longworth S, Kreiswirth BN, and Vinnard C. Deaths Related to Nontuberculous Mycobacterial Infections in the United States, 1999-2014. Poster Presentation, IDSA Meeting. October 2016.

${ }^{202}$ Trieste L and Tuchetti G. Cost for Tuberculosis Care in Developed Countries: Which Data for an Economic Evaluation? J Rheumatol Suppl. 2014 May;91:83-85.

${ }^{203}$ Tanimura T, Jaramillo W, Weil D, Ravilglione M, Loennroth K. Financial burden for tuberculosis patients in low- and middle-income countries: a systematic review. Eur Respir J 2014 Jun;43(6):1763-1775.

${ }^{204}$ Tanimura T, Jaramillo W, Weil D, Ravilglione M, Loennroth K. Financial burden for tuberculosis patients in low- and middle-income countries: a systematic review. Eur Respir J 2014 Jun;43(6):1763-1775.

205 Tanimura T, Jaramillo W, Weil D, Ravilglione M, Loennroth K. Financial burden for tuberculosis patients in low- and middle-income countries: a systematic review. Eur Respir J 2014 Jun;43(6):1763-1775.

${ }^{206}$ Nair DM, George A, Chacko KT. Tuberculosis in Bombay: new insights from poor urban patients. Health Policy Plan. 1997 Mar;12(1):77-85.

${ }^{207}$ Rajeswari R, Balasubramanian R, Muniyandi M, Geetharamani S, Thresa X, Venkatesan P. Socio-economic impact of tuberculosis on patients and family in India. Int J Tuberc Lung Dis.1999 Oct;3(10):869877.

${ }^{208}$ Paton NI, Castello-Branco LR, Jennings G, Ortiago-de-Sampaio MB, Elia M, Costa S, Griffin GE. Impact of tuberculosis on the body composition of HIV-infected men in Brazil. J Acquir Immune Defic Syndr Hum Retrovirol. 1999 Mar 1;20(3):265-271.

209 Paton NI, Castello-Branco LR, Jennings G, Ortiago-de-Sampaio MB, Elia M, Costa S, Griffin GE. Impact of tuberculosis on the body composition of HIV-infected men in Brazil. J Acquir Immune Defic Syndr Hum Retrovirol. 1999 Mar 1;20(3):265-271.

${ }^{210}$ Macallan DC, McNurlan MA, Kurpad AV, de Souza G, Shetty PS, Calder AG, Griffin GE. Whole body protein metabolism in human pulmonary tuberculosis and undernutrition: evidence for anabolic block in tuberculosis. Clin Sci (Lond.).1998 Mar; 94(3)321-331. 
${ }^{211}$ Paton NI, Ng YM. Body composition studies in patients with wasting associated with tuberculosis. Nutrition. 2006 Mar;22(3):245-251.

212 Harries AD, Nkhoma WA, Thompson PJ, Nyangulu DS, Wirima JJ. Nutritional status in Malawian patients with pulmonary tuberculosis and response to chemotherapy. Eur J Clin Nutr. 1988 May;42(5):445-450.

${ }^{213}$ Paton NI, Ng YM. Body composition studies in patients with wasting associated with tuberculosis. Nutrition. 2006 Mar;22(3):245-251.

${ }^{214}$ PrayGod G, Range N, Faurholt-Jepsen D, Jeremiah K, FaurholtJepsen M, Aabye MG, Magnussen P, Changalucha J, Andersen AB, Wells $\mathrm{JC}$, Friis $\mathrm{H}$. Sex, smoking, and socioeconomic status are associated with body composition among tuberculosis patients in a deuterium dilution cross-sectional study in Mwanza, Tanzania. J Nutr. 2013 May;143(5):735741.

${ }^{215}$ Zachariah R, Spielmann MP, Harries AD, Salaniponi FM. Moderate to severe malnutrition in patients with tuberculosis is a risk factor associated with early death. Trans R Soc Trop Med Hyg. 2002 May-Jun;96(3):291294

${ }^{216}$ Peabody JW, Shimkhada R, Tan C Jr, Luck J. The burden of disease, economic costs and clinical consequences of tuberculosis in the Philippines. Health Policy Plan. 2005 Nov;20(6):347-353.

${ }^{217}$ Murray CJL. Results of directly observed short course chemotherapy in 112,842 Chinese patients with smear positive tuberculosis. Lancet. 1996:347(8998);358-362.

${ }^{218}$ Tang S and Squire SB. What lessons can be drawn from tuberculosis (TB) Control in China in the 1990s? An analysis from a health system perspective. Health Policy. 2005 Apr:72(1);93-104.

${ }^{219}$ Long Q, Smith H, Zhang T, Tang S and Garner P. Patient medical costs for tuberculosis treatment and impact on adherence in China: a systematic review. BMC Public Health. 2011 May 26;11:393.

${ }^{220}$ Barter DM, Agboola SO, Murray MB and Baeringhausen T. Tuberculosis and poverty: the constribution of patient costs in subSaharan Africa-a systematic review. BMC Public Health. 2012 Nov $14 ; 12: 980$.

${ }^{221}$ Mauch V, Bonsu F, Gyapong M, Awini E, Suarez P, Marcelino B, Melgen RE, Lönnroth K, Nhung NV, Hoa NB, Klinkenberg E. Free tuberculosis diagnosis and treatment are not enough: patient cost evidence from three continents. Int J Tuberc Lung Dis. 2013 
Mar;17(3):381-387.

${ }^{222}$ WHO. World Health Report 2010. Health Systems Financing-the Path to Universal Coverage. Geneva, World Health Organization, 2010.

${ }^{223}$ WHO. Eliminating the Catastrophic Economic Burden of TB: Universal Health Coverage and Social Protection Opportunities. Meeting Report from a World Health Organization Consultation to Inform the Post-2015 Global TB Strategy Geneva, World Health Organization, 2013.

${ }^{224}$ WHO. World Tuberculosis Report Supplement: Countdown to 2015. WHO;Geneva, Switzerland: 2013.

${ }^{225}$ Martinson NA, Karstaedt A, Venter MD, Omar T, King P, Mbengo T, Marais E, McIntyre J, Chaisson RE, Hale M. Causes of death in hospitalized adults with a premortem diagnosis of tuberculosis: an autopsy study. AIDS. 2007 Oct 1;21(15):2043-50.

${ }^{226}$ Cox JA, Lukande RL, Nelson AM, Mayanja-Kizza H, Colebunders R, Van Marck E, Manabe YC. An autopsy study describing causes of death and comparing clino-pathological findings among hospitalized patients in Kampala, Uganda. PLoS One. 2012;7(3):e33685.

${ }^{227}$ Pooran A, Pieterson E, Davids $\mathrm{M}$ et al. What is the cost of diagnosis and management of drug resistant tuberculosis in South Africa? 2013. PLoS One;8(1):e54587.

${ }^{228}$ Walwyn DR. Determining quantitative targets for public funding of tuberculosis research and development. Health Res Policy Syst. 2013 Mar 8;11(1):10.

${ }^{229}$ STOP TB website. New Diagnostics working Group. Available at: www.stoptb.org/wg/new_diagnostics/. Accessed July 22, 2016.

230 UNITAID. Tuberculosis diagnostics technology landscape report. WHO; Geneva. Switzerland: 2012.

${ }^{231}$ Molicotti P, Bua A, and Zanetti S. Cost-effectiveness in the diagnosis of tuberculosis: choices in developing countries. J Infect Dev Ctries. 2014 Jan 15:8(1):24-38.

${ }^{232}$ Chihota VN, Grant AD, Fielding K, Ndibongo B, van Zyl A, Muirhead D. Liquid vs solid culture in a resource-contrained setting. 2010 Aug. Int Tuberc Lun Dis;14:1024-1031.

${ }^{233}$ Molicotti P, Bua A, and Zanetti S. Cost-effectiveness in the diagnosis of tuberculosis: choices in developing countries. J Infect Dev Ctries. 2014 
Jan 15:8(1):24-38.

${ }^{234}$ Pantoja A, Kirk SV Denkinger CM. Costs of Novel Tuberculosis Diagnostics-Will Countries Be Avle to Afford It? J Infect Dis. 2015 Apr 1;211 Suppl 2:S67-77.

${ }^{235}$ Lu C, Liu Q, Sarma A, Fitzpatrick C, Falzon D Mitnick CD. PLOS ONE. Feb 2013;8(2):e56074.

${ }^{236}$ Sohn H, Minion J, Albert H, Dheda K and Pai M. TB diagnostics tests: how do we figure their costs? Expert Rev Anti Infect Ther. 2009 Aug;7(6):723-733.

${ }^{237}$ Areeshi MY, Bisht SC, Mandal RK, Haque S. Prevalence of drug resistance in clinical isolates of tuberculosis from GCC: a literature review from January 2002 to March 2013. J Infect Dev Ctries. 2014 Sep 12;8(9):1137-1147.

${ }^{238}$ Mishra R, Shukla P, Huang W, Hu N. Gene mutations in Mycobacterium tuberculosis: multidrug-resistant TB as an emerging global public health crisis. Tuberculosis (Edinb). 2015 Jan;95(1):1-5.

${ }^{239}$ Mishra R, Shukla P, Huang W, Hu N. Gene mutations in Mycobacterium tuberculosis: multidrug-resistant TB as an emerging global public health crisis. Tuberculosis (Edinb). 2015 Jan;95(1):1-5.

${ }^{240}$ WHO. Multidrug and extensively drug-resistant TB (M/XDR-TB):2010 global report on surveillance and response. Geneva, Switzerland, 2010

${ }^{241}$ WHO. Guidelines for the programmatic management of drug-resistant tuberculosis: emergency update 2008. Geneva, Switzerland, 2008

${ }^{242}$ WHO. Travel, foreign policy, diplomacy and health. Tuberculosis (TB). Geneva, Switzerland.

${ }^{243}$ Marks SM, Flood J, Seaworth B, Hirsch-Moverman Y, Armstrong L, Mase S, Salcedo K, Oh P, Graviss EA, Colson PW, Armitige L, Revuelta M, Sheeran K; TB Epidemiologic Studies Consortium. Treatment practices, outcomes and costs of multi-drug resistant and extensively drug resistant tuberculosis, United States 2005-2007. Emerg Infect Dis. 2014 May;20(5):812-821.

${ }^{244}$ Rouzier VA, Oxlade O, Verduga R, Gresely L, Menzies D. Patient and family costs associated with tuberculosis, including multidrug-resistant tuberculosis, in Ecuador. Int J Tuberc Lung Dis. 2010 Oct;14(10):13161322. 
${ }^{245}$ Pooran A, Pieterson E, Davids $M$ et al. What is the cost of diagnosis and management of drug resistant tuberculosis in South Africa? 2013. PLoS One;8(1):e54587.

${ }^{246}$ WHO. Eliminating the Catastrophic Economic Burden of TB: Universal Health Coverage and Social Protection Opportunities. Meeting Report from a World Health Organization Consultation to Inform the Post-2015 Global TB Strategy Geneva, World Health Organization, 2013.

${ }^{247}$ Fitzpatrick A and Floyd K. A systematic review of the cost and cost effectiveness of treatment for multi-drug-resistant tuberculosis.

Pharmaeconomics. 2012 Jan;30(1):63-80.

${ }^{248}$ Restrepo, BI. Convergence of the tuberculosis and diabetes epidemics: renewal of old acquaintances. Clin Infect Dis. 2007 Aug 15;45(4):436-438.

${ }^{249}$ Golden MP, Vikram HR. Extrapulmonary tuberculosis: an overview. Am Fam Physician. 2005 Nov 1;72(9):1761-1768.

${ }^{250}$ WHO website. Tuberculosis Fact sheet $\mathrm{N}^{\circ} 104$. World Health Organization. October 2015. Available at:

http://www.who.int/mediacentre/factsheets/fs104/en/. Accessed December $1,2015$.

${ }^{251}$ Grant, Jessica. (2011) The Determinants of Tuberculosis Transmission in Indigenous People in Canada and New Zealand (Master's Thesis). Retrieved from ecommons.usask.ca

${ }^{252}$ Hargreaves S, Carballo M, Friedland JS. Screening immigrants for tuberculosis: where's next? Lancet Infect Dis. 2009 Mar;9(3):139-140

${ }^{253}$ Liu Y, Weinberg MS, Ortega LS, Painter JA, Maloney SA. Overseas screening for tuberculosis in U.S.-bound immigrants and refugees. N Engl J Med.2009 June 4;360(23):103-106.

${ }^{254}$ Health Protection Agency. Tuberculosis in the UK: annual report on tuberculosis surveillance and control in the UK 2009. London, Health Protection Agency Centre for Infections, 2009.

${ }^{255}$ European Centre for Disease Prevention and Control/WHO Regional Office for Europe. Tuberculosis surveillance in Europe 2013. Stockholm: European Centre for Disease Prevention and Control; 2015.

${ }^{256}$ European Centre for Disease Prevention and Control/ WHO Regional Office for Europe. Tuberculosis surveillance in Europe 2008. Stockholm, European Centre for Disease Prevention and Control, 2010. 
${ }^{257}$ European Centre for Disease Prevention and Control/WHO Regional Office for Europe. Tuberculosis surveillance in Europe 2013. Stockholm: European Centre for Disease Prevention and Control; 2015.

${ }^{258}$ Alami NN, Yuen CM, Miramontes R, Pratt R, Price SF, Navin TR; Centers for Disease Control and Prevention (CDC). Trends in tuberculosis-United States, 2013. MMWR 2014 Mar 21;63(11):229-233.

${ }^{259}$ Posey DL, Naughton MP, Willacy EA, Russell M, Olson CK, Godwin CM, McSpadden PS, White ZA, Comans TW, Ortega LS, Guterbock M, Weinberg MS, Cetron MS; Centers for Disease Control and Prevention (CDC). Implementation of new TB screening requirements for U.S.-bound immigrants and refugees - 2007-2014. MMWR Morb Mortal Wkly Rep. 2014 Mar 21;63(11):234-236.

${ }^{260}$ Pareek M, Greenaway C, Noori T, Munoz J, Zenner D. The impact of migration on tuberculosis epidemiology and control in high-income countries: a review. BMC Med. 2016 Mar 23;14:48.

${ }^{261}$ Health Protection Agency. Tuberculosis in the UK: annual report on tuberculosis surveillance and control in the UK 2009. London, Health Protection Agency Centre for Infections, 2009.

${ }^{262}$ Pareek M, Greenaway C, Noori T, Munoz J, Zenner D. The impact of migration on tuberculosis epidemiology and control in high-income countries: a review. BMC Med. 2016 Mar 23;14:48

${ }^{263}$ European Centre for Disease Prevention and Control/WHO Regional Office for Europe. Tuberculosis surveillance in Europe 2013. Stockholm: European Centre for Disease Prevention and Control; 2015.

${ }^{264}$ Pareek M, Greenaway C, Noori T, Munoz J, Zenner D. The impact of migration on tuberculosis epidemiology and control in high-income countries: a review. BMC Med. 2016 Mar 23;14:48.

265 United Nations Department of Economic and Social Affairs Population Division. International Migration Report 2013. Geneva: United Nations; 2013

${ }^{266}$ Amnesty International website. Syria's Refugee Crisis in Numbers. Feburary 3, 2016. Accessed June 28, 2016, 2016. Available at: https://www.amnesty.org/en/.../syrias-refugee-crisis-in-numbers/

${ }^{267}$ Eurostat Statistics Explained website. Asylum Statistics. April 2016. Accessed June 28, 2016, 2016. Available at ec.europa.eu

${ }^{268}$ Sengupta S. U.S. Has Taken In Less Than a Fifth of Pledged Syrian 
Refugees. May 10, 2016. Available at www.nytimes.com

${ }^{269} 5$ things to know about Canada's Syrian refugee program: Canada is officially resettling more Syrian refugees than many other countries. February 29, 2016. Available at www.cbc.ca.

${ }^{270}$ Posey DL, Naughton MP, Willacy EA, Russell M, Olson CK, Godwin CM, McSpadden PS, White ZA, Comans TW, Ortega LS, Guterbock M, Weinberg MS, Cetron MS; Centers for Disease Control and Prevention (CDC). Implementation of new TB screening requirements for U.S.-bound immigrants and refugees - 2007-2014. MMWR Morb Mortal Wkly Rep. 2014 Mar 21;63(11):234-6.

${ }^{271}$ Posey DL, Naughton MP, Willacy EA, Russell M, Olson CK, Godwin CM, McSpadden PS, White ZA, Comans TW, Ortega LS, Guterbock M, Weinberg MS, Cetron MS; Centers for Disease Control and Prevention (CDC). Implementation of new TB screening requirements for U.S.-bound immigrants and refugees - 2007-2014. MMWR Morb Mortal Wkly Rep. 2014 Mar 21;63(11):234-236.

272 Davies PD. The role of DOTS in tuberculosis treatment and control. Am J Respir Med. 2003;2(3):203-209.

${ }^{273}$ Pinet G. Good practice in legislation and regulations for TB control: an indicator of political will. Geneva, World Health Organization, 2001

${ }^{274}$ Walley JD, Khan MA, Newell JN, Khan MH. Effectiveness of the direct observation component of DOTS for tuberculosis: a randomised controlled trial in Pakistan. Lancet. 2001 Mar 2;357(9257):664-669.

${ }^{275}$ Parida A, Bairy KL, Chogtu B, Magazine R, Vidyasagar S. Comparison of Directly Observed Treatment Short Course (DOTS) with SelfAdministered Therapy in Pulmonary Tuberculosis in Udupi District of Southern India. J Clin Diagn Res. 2014 Aug;8(8):HC29-31.

${ }^{276}$ Posey DL, Naughton MP, Willacy EA, Russell M, Olson CK, Godwin CM, McSpadden PS, White ZA, Comans TW, Ortega LS, Guterbock M, Weinberg MS, Cetron MS; Centers for Disease Control and Prevention (CDC). Implementation of new TB screening requirements for U.S.-bound immigrants and refugees - 2007-2014. MMWR Morb Mortal Wkly Rep. 2014 Mar 21;63(11):234-236.

${ }^{277}$ Mor Z, Leventhal A, Diacon AH, Finger R, Schoh OD. Tuberculosis screening in immigrants from high-prevalence countries: Interview first or chest radiograph first? A pro/con debate. Respiratory. 2013 Apr;18(3):432438. 
${ }^{278}$ Arshad S, Bavan L, Gajari K et al. Active screening at entry for tuberculosis among new immigrants: a systematic review and metaanalysis. Eur Respir J. 2010 Jun;35(6):1336-1345.

${ }^{279}$ Zellweger JP, Heinzer R, Touray M et al. Intra-observer and overall agreement in the radiological assessment of tuberculosis. Int J Tuberc. Lung Dis. 2006 Oct;10(10):1123-1126

${ }^{280}$ Mathez C, Bangala Y, Bady P, et al. Active screening for pulmonary tuberculosis among immigrants by chest $\mathrm{x}$-ray at the Swiss border. Swiss Med Wkly. 2007 Nov 17;137(45-46):649-654.

${ }^{281}$ European Centre for Disease Prevention and Control/WHO Regional Office for Europe. Tuberculosis surveillance in Europe 2013. Stockholm: European Centre for Disease Prevention and Control; 2015.

${ }^{282}$ Schneeberger Gesiler E, Helbing P, Zellweger JP et al. Screening for tuberculosis in asylum seekers: comparison of chest radiography with an interview-based system. Int J Tuberc Lung Dis. 2010 Nov;14(11):13881384.

${ }^{283}$ World Health Organisation. Guidelines on the management of latent tuberculosis infection. Geneva: World Health Organisation; 2014.

${ }^{284}$ Pareek M, Greenaway C, Noori T, Munoz J, Zenner D. The impact of migration on tuberculosis epidemiology and control in high-income countries: a review. BMC Med. 2016 Mar 23;14:48.

${ }^{286}$ Wingate LT, Coleman MS, de la Motte Hurst C, Semple M, Zhou W, Cetron MS, Painter JA. A cost-benefit analysis of a proposed overseas refugee latent tuberculosis infection screening and treatment program. BMC Public Health. 2015 Dec 1;15:1201.

${ }^{287}$ Kardos M, Kimball AB. Time for a change? Updated guidelines using interferon gamma release assays for detection of latent tuberculosis infection in the office setting. J Am Acad Dermatol. 2012 Jan;66(1):148152.

${ }^{288}$ WHO website. BCG Vaccine: WHO position paper. Weekly epidemiological record 4 (79): 25-40. Jan 23, 2014. Available at who.org. Accessed July 1, 2016.

${ }^{289}$ WHO (2004). WHO Position Paper on BCG Vaccination.Geneva: WHO.

${ }^{290}$ WHO website. BCG Vaccine: WHO position paper. Weekly epidemiological record 4 (79): 25-40. Jan 23, 2014. Available at who.org. 
Accessed July 1, 2016.

${ }^{291}$ Lienhardt C, Fielding K, Hane AA, Niang A, Ndao CT, Karam F, et al. Evaluation of the prognostic value of IFN-y release assay and tuberculin skin test in household contacts of infectious tuberculosis cases in Senegal. PLos Med 2010 May; 5(5):1-10.

${ }^{292}$ Caglayan V, Ak O, Dabak G, Damadoglu E, Ketenci B, Ozdemir M, Ozer S, Saygı A. Comparison of tuberculin skin testing and QuantiFERON-TB gold-In Tube test in health care workers. Tuberk Toraks 2011; 59:43-47.

${ }^{293}$ Ferreira TF, Matsuoka Pda F, Santos AM, Caldas Ade J. Diagnosis of latent Mycobacterium tuberculosis infection: tuberculin test versus interferon-gamma release. Rev Soc Bras Med Trop. 2015 NovDec;48(6):724-730.

${ }^{294}$ Mathad JS, Bhosale R, Sangar V, Mave V, Gupte N, Kanade S, Nangude A, Chopade K, Suryavanshi N, Deshpande P, Kulkarni V, Glesby MJ, Fitzgerald D, Bharadwaj R, Sambarey P, Gupta A. Pregnancy differentially impacts performance of latent tuberculosis diagnostics in a high-burden setting. PLoS One. 2014 Mar 21;9(3):e92308.

295 Kardos M, Kimball AB. Time for a change? Updated guidelines using interferon gamma release assays for detection of latent tuberculosis infection in the office setting. J Am Acad Dermatol. 2012 Jan;66(1):148152.

${ }^{296}$ Pareek M, Bond M, Shorey J, Seneviratne S, Guy M, et al. (2012) Community- based evaluation of immigrant tuberculosis screening using interferon gamma release assays and tuberculin skin testing: observational study and economic analysis. Thorax. 2013 Mar;68(3):230239.

297 O'Shea MK, Fletcher TE, Beeching NJ, Dedicoat M, Spence D, McShane $\mathrm{H}$. Tuberculin skin testing and treatment modulates interferongamma release assay results for latent tuberculosis in migrants. PLoS One. 2014 May 9;9(5):e97366.

${ }^{298}$ Mussert, Linda. (2008). Cost effectiveness of different scenarios for diagnosing and treatment of LTBI of immigrant close contacts in the Netherlands. (Master's Thesis)

299 Wyss LL, Alderman MK. Using theory to interpret beliefs in migrants diagnosed with latent TB. Online J Issues Nurs. 2006 Nov 16;12(1):7.

${ }^{300}$ Pareek M, Greenaway C, Noori T, Munoz J, Zenner D. The impact of 
migration on tuberculosis epidemiology and control in high-income countries: a review. BMC Med. 2016 Mar 23;14:48.

301 Department of Homeland Security. Estimates of the Unauthorized Immigrant Population Residing in the United States: January 2012 dhs.gov

${ }^{302}$ Buijtels, P. (2007) Clinical relevance of non-tuberculosis mycobacteria in Zambia (Doctoral dissertation). Retrieved from: repub.eur.nl. ISBN 97890-8559-154-2

303 López-Varela E, García-Basteiro AL, Santiago B, Wagner D, van Ingen J, Kampmann B. Non-tuberculous mycobacteria in children: muddying the waters of tuberculosis diagnosis. Lancet Respir Med. 2015 Mar;3(3):244-56.

304 Buijtels, Patricia. (2007) Clinical relevance of non-tuberculosis mycobacteria in Zambia (Doctoral dissertation). Retrieved from: repub.eur.nl. ISBN 978-90-8559-154-2

${ }^{305}$ Maiga M, Siddiqui S, Diallo S, et al: Failure to recognize nontuberculous mycobacteria leads to misdiagnosis of chronic pulmonary tuberculosis. PLoS One 2012;7(5):e36902

${ }^{306}$ Tabarsi P, Baghaei P, Farnia P, Mansouri N, Chitsaz E, Sheikholeslam F, Marjani M, Rouhani N, Mirsaeidi M, Alipanah N, Amiri M, Masjedi MR, Mansouri D. Nontuberculous mycobacteria among patients who are suspected for multidrug-resistant tuberculosis-need for earlier identification of nontuberculous mycobacteria. Am J Med Sci. 2009 Mar;337(3):182-184.

${ }^{307}$ Pang Y, Zhou Y, Wang S, Tan Y, Yue J, Zhao B, Wang L, Zhao Y, Kam $\mathrm{KM}$. Rapid molecular identification of mycobacterial species in positive culture isolates using the biochip test. Int J Tuberc Lung Dis. 2011 Dec;15(12):1680-1685.

${ }^{308}$ Tabarsi P, Baghaei P, Farnia P, Mansouri N, Chitsaz E, Sheikholeslam F, Marjani M, Rouhani N, Mirsaeidi M, Alipanah N, Amiri M, Masjedi MR, Mansouri D. Nontuberculous mycobacteria among patients who are suspected for multidrug-resistant tuberculosis-need for earlier identification of nontuberculous mycobacteria. Am J Med Sci. 2009 Mar;337(3):182-184.

${ }^{309}$ Shahraki AH, Heidarieh P, Bostanabad SZ, Khosravi AD, Hashemzadeh M, Khandan S, Biranvand M, Schraufnagel DE, Mirsaeidi M. Eur J Intern Med. "Multidrug-resistant tuberculosis" may be nontuberculous mycobacteria. Eur J Intern Med. 2015 May;26(4):279-284. 
${ }^{310}$ Aliyu G, El-Kamary SS, Abimiku A, Brown C, Tracy K, Hungerford L, Blattner W. et al. Prevalence of non-tuberculous mycobacterial infections among tuberculosis suspects in Nigeria. PloS one. 2013 May 9;8(5):e63170.

${ }^{311}$ Marras TK. Chedore P, Ying AM, Jamieson F. Isolation prevalence of pulmonary non-tubercolosis mycobacteria in Ontario, 1997-2003. Thorax. 2007 Aug.62(8);661-666.

${ }^{312}$ Brode SK, Daley CL, Marras TK. The epidemiologic relationship between tuberculosis and non-tuberculous mycobacterial disease: A systematic review. Int J Tuberc Lung Dis. 2014 Nov;18(11):1370-1377.

${ }^{313}$ Arastéh KN, Cordes C, Ewers M, Simon V, Dietz E, Futh UM, Brockmeyer NH, L'age MP. HIV-related nontuberculous mycobacterial infection: incidence, survival analysis and associated risk factors. Eur J Med Res. 2000 Oct 30;5(10):424-430.

${ }^{314}$ Ballarino GJ, Olivier KN, Claypool RJ, Holland SM, Prevots DR. Pulmonary nontuberculous mycobacterial infections: antibiotic treatment and associated costs. Respir Med. 2009 Oct;103(10):1448-1455.

315 Leber A and Marras TK. The cost of medical management of pulmonary nontuberculous mycobacterial disease in Ontario, Canada. Eur Respir J. 2011 May;37(5):1158-65.

${ }^{316}$ Leber A and Marras TK. The cost of medical management of pulmonary nontuberculous mycobacterial disease in Ontario, Canada. Eur Respir J. 2011 May;37(5):1158-1165.

317 Strollo SE, Adjemian J, Adjemian MK and Prevots DR. The burden of pulmonary Nontuberculous mycobacterial disease in the United States. Ann Am Thorac Soc. 2015 Oct;12(10):1458-1464.

${ }^{318}$ Collier SA, Stockman LJ, Hicks LA, Garrison LE, Zhou FJ, Beach MJ. Direct healthcare costs of selected diseases primarily or partially transmitted by water. Epidemiol Infect 2012 Nov;140(11):2003-2013.

${ }^{319}$ Google Currency Converter. Available at:

https://www.google.com/search?client=safari\&rls=en\&q=currency+convert er+google\&ie=UTF-8\&oe=UTF-8 Accessed: November 17, 2016.

${ }^{320}$ US Bureau of Labor and Statistics website. CPI Inflation Calculator. Available at: http://data.bls.gov/cgi-bin/cpicalc.pl. Accessed November 17, 2016. 
${ }^{321}$ Healthcare Cost and Utilization Project website. Overview of National Inpatient Sample. Available at: http://www.hcup-

us.ahrq.gov/nisoverview.jsp. Accessed on Jan 2, 2015.

${ }^{322}$ Healthcare Cost and Utilization Project website. Available at:

http://hcupnet.ahrq.gov/HCUPnet.jsp? Id=C73A51CC022179F8\&Form=Sel PAT\&GoTo=ZIPinc\&JS=Y\&Defld= Accessed on Jan 2, 2015.

${ }^{323}$ Mirsaeidi M, Farshidpour M, Ebrahimi G, Aliberti S, Falkinham JO, 3rd. Management of nontuberculous mycobacterial infection in the elderly. Eur J Intern Med. 2014 Apr;25(4):356-363.

${ }^{324}$ Mirsaeidi M, Allen MB, Ebrahimi G, and Falkinham JO. Highlight on Advances in Nontuberculous Mycobacterial Disease in North America. Biomed Res Int. 2014;2014:919474.

${ }^{325}$ Thomson RM; NTM working group at Queensland TB Control Centre and Queensland Mycobacterial Reference Laboratory. Changing epidemiology of pulmonary nontuberculous mycobacteria infections. Emerg Infect Dis. 2010 Oct;16(10):1576-1583.

${ }^{326}$ Mirsaeidi M, Machado RF, Garcia JG, Schraufnagel DE. Nontuberculous mycobacterial disease mortality in the United States, 1999-2010: a population-based comparative study. PloS one. 2014;9(3):e91879.

${ }^{327}$ Mezochow A, Hamilton K, Longworth S, Kreiswirth BN, and Vinnard C. Deaths Related to Nontuberculous Mycobacterial Infections in the United States, 1999-2014. Poster Presentation, IDSA Meeting. October 2016.

${ }^{328}$ Adjemian J, Olivier KN, Seitz AE, Falkinham JO, III, Holland SM, Prevots DR. Spatial clusters of nontuberculous mycobacterial lung disease in the United States. Am J Respir Crit Care Med. 2012 Sep 15;186(6):553-558.

${ }^{329}$ Hoefsloot W, van Ingen J, Andrejak C, et al. The geographic diversity of nontuberculous mycobacteria isolated from pulmonary samples: an NTM-NET collaborative study. European Respiratory Journal. 2013 Dec. 42(6);1604-1613.

${ }^{330}$ Trochim WM, Cabrera DA, Milstein B, Gallagher RS, and Leischow SJ. Practical challenges of systems thinking and modeling in public health. Am J Public Health. 2006 Mar;96(3):538-546.

${ }^{331}$ Milstein B, Homer J, and Hirsch G. Analyzing national health reform strategies with a dynamic simulation model. Am J Public Health. 2010 
May;100(5):811-819.

${ }^{332}$ Trochim WM, Cabrera DA, Milstein B, Gallagher RS, and Leischow SJ. Practical challenges of systems thinking and modeling in public health. Am J Public Health. 2006 Mar;96(3):538-546.

${ }^{333}$ Milstein B. 2008. Hygeia's Constellation: Navigating Health Futures in a Dynamic and Democratic World. Atlanta: Centers for Disease Control and Prevention, Syndemics Prevention Network.

${ }^{334}$ Trochim WM, Cabrera DA, Milstein B, Gallagher RS, and Leischow SJ. Practical challenges of systems thinking and modeling in public health. Am J Public Health. 2006 Mar;96(3):538-546.

335 Potash PJ and Heinbokel JF, Unleashing the Revolutionary Implications of a System Dynamics Education Using a Ladder of Engagement. Proceedings of the 23rd International Conference of the System Dynamics Society, Boston, July, 2005.

${ }^{336}$ Homer JB and Hirsch GB. System dynamics modeling for public health: background and opportunities. Am J Public Health. 2006 Mar;96(3):452458.

${ }^{337}$ Trochim WM, Cabrera DA, Milstein B, Gallagher RS, and Leischow SJ. Practical challenges of systems thinking and modeling in public health. Am J Public Health. 2006 Mar;96(3):538-546.

${ }^{338}$ Leischow SJ, Best A, Trochim WM, Clark PI, Gallagher S. Marcus SE, Matthews E. Systems thinking to improve the public's health. Am J Prev Med. 2008 Aug; 35(2 Suppl):S196-S203.

${ }^{339}$ Homer JB and Hirsch GB. System dynamics modeling for public health: background and opportunities. Am J Public Health. 2006 Mar;96(3):452458.

${ }^{340}$ Tian Y, Alawami F, Al-Azem A, Osgood N, Hoeppner V, Dutchyn C. A System Dynamics Model of Tuberculosis Diffusion with Respect to Contact Tracing Investigation. 2011 Winter Simulation Conference

${ }^{341}$ WHO. World Health Report 2010. Health Systems Financing-the Path to Universal Coverage. Geneva, World Health Organization, 2010.

${ }^{342}$ WHO. Eliminating the Catastrophic Economic Burden of TB: Universal Health Coverage and Social Protection Opportunities. Meeting Report from a World Health Organization Consultation to Inform the Post-2015 Global TB Strategy Geneva, World Health Organization, 2013. 
${ }^{343}$ Long Q, Smith H, Zhang T, Tang S and Garner P. Patient medical costs for tuberculosis treatment and impact on adherence in China: a systematic review. BMC Public Health. 2011 May 26;11:393.

${ }^{344}$ Mishra R, Shukla P, Huang W, Hu N. Gene mutations in Mycobacterium tuberculosis: multidrug-resistant TB as an emerging global public health crisis. Tuberculosis (Edinb). 2015 Jan;95(1):1-5.

${ }^{345}$ Mauch V, Bonsu F, Gyapong M, Awini E, Suarez P, Marcelino B, Melgen RE, Lönnroth K, Nhung NV, Hoa NB, Klinkenberg E. Free tuberculosis diagnosis and treatment are not enough: patient cost evidence from three continents. Int J Tuberc Lung Dis. 2013 Mar;17(3):381-387.

${ }^{346}$ Alami NN, Yuen CM, Miramontes R, Pratt R, Price SF, Navin TR; Centers for Disease Control and Prevention (CDC). Trends in tuberculosis—United States, 2013. MMWR 2014;63(11):229-233.

${ }^{347}$ Mor Z, Leventhal A, Diacon AH, Finger R, Schoh OD. Tuberculosis screening in immigrants from high-prevalence countries: Interview first or chest radiograph first? A pro/con debate. Respiratory. 2013 Apr;18(3):432438.

${ }^{348}$ Schneeberger Gesiler E, Helbing P, Zellweger JP et al. Screening for tuberculosis in asylum seekers: comparison of chest radiography with an interview-based system. Int J Tuberc Lung Dis. 2010 Nov;14(11):13881384.

349 Pareek M, Greenaway C, Noori T, Munoz J, Zenner D. The impact of migration on tuberculosis epidemiology and control in high-income countries: a review. BMC Med. 2016 Mar 23;14:48.

${ }^{350}$ Runyon EH. Anonymous mycobacteria in pulmonary disease. The Medical clinics of North America.January 1959;43 (1): 273-290.

${ }^{351}$ Cassidy PM, Hedberg K, Saulson A, McNelly E and Winthrop KL. Nontuberculous prevalence and risk factors: a changing epidemiology. Clin Infect Dis. 2009 Dec 15;49(12).e124-129.

${ }^{352}$ Cassidy PM, Hedberg K, Saulson A, McNelly E and Winthrop KL. Nontuberculous prevalence and risk factors: a changing epidemiology. Clin Infect Dis. 2009 Dec 15;49(12).e124-129.

${ }^{353}$ Kasperbauer $\mathrm{S}$ and Huitt G. Management of extrapulmonary nontuberculous mycobacteria infections. Semin Respir Crit Care Med. 2013 Feb;34(1):143-150. 
${ }^{354}$ Maiga M, Siddiqui S, Diallo S, Diarra B, Traoré B, Shea YR, Zelazny AM, Dembele BP, Goita D, Kassambara H, Hammond AS, Polis MA, Tounkara A. Failure to recognize nontuberculous mycobacteria leads to misdiagnosis of chronic pulmonary tuberculosis. PLoS One 2012;7(5): e36902

${ }^{355}$ Tabarsi P, Baghaei P, Farnia P, Mansouri N, Chitsaz E, Sheikholeslam F, Marjani M, Rouhani N, Mirsaeidi M, Alipanah N, Amiri M, Masjedi MR, Mansouri D. Nontuberculous mycobacteria among patients who are suspected for multidrug-resistant tuberculosis-need for earlier identification of nontuberculous mycobacteria. Am J Med Sci. 2009 Mar;337(3):182-184.

${ }^{356}$ Pang Y, Zhou Y, Wang S, Tan Y, Yue J, Zhao B, et al. Rapid molecular identification of mycobacterial species in positive culture isolates using the biochip test. Int J Tuberc Lung Dis. 2011 Dec;15(12):1680-1685.

${ }^{357}$ Cassidy PM, Hedberg K, Saulson A, McNelly E and Winthrop KL. Nontuberculous prevalence and risk factors: a changing epidemiology. Clin Infect Dis. 2009 Dec 15;49(12):e124-129.

${ }^{358}$ Kartalija M, Ovrutsky AR, Bryan CL, Pott GB, Fantuzzi G, Thomas J, Strand MJ, Bai X, Ramamoorthy P, Rothman MS, Nagabhushanam V, McDermott M, Levin AR, Frazer-Abel A, Giclas PC, Korner J, Iseman MD, Shapiro L, Chan ED. Patients with nontuberculous mycobacterial lung disease exhibit unique body and immune phenotypes. Am J Respir Crit Care Med. 2013 Jan 15;187(2):197-205.

${ }^{359}$ Chan ED, Iseman MD. Slender, older women appear to be more susceptible to nontuberculous mycobacterial lung disease. Gender Medicine. 2010 Feb;7(1):5-18.

${ }^{360}$ Leung JM, Olivier KN. Nontuberculous mycobacteria: The changing epidemiology and treatment challenges in cystic fibrosis. Curr Opin Pulm Med. 2013 Nov;19(6):662-669.

${ }^{361}$ Wiener JM, Tilly J Population ageing in the United States of America: implications for public programmes. Int J Epidemiol. 2002 Aug; 31(4):776781.

${ }^{362}$ Rynning E. The ageing populations of Europe--implications for health systems and patients' rights. Eur J Health Law. 2008 Sep;15(3):297-306.

${ }^{363}$ Andréjak C, Thomsen VØ, Johansen IS, Riis A, Benfield TL, Duhaut P, Sørensen HT, Lescure F-X, Thomsen RW. Nontuberculous pulmonary mycobacteriosis in Denmark: incidence and prognostic factors. Am J 
Respir Crit Care Med. 2010 Mar 1;181(5):514-521.

${ }^{364}$ Piersimoni C. Nontuberculous mycobacteria infection in solid organ transplant recipients. European Journal of Clinical Microbiology and Infectious Diseases. 2012;31(4):397-403.

${ }^{365}$ Knoll BM, Kappagoda S, Gill RR., Goldberg HJ, Boyle K, Baden LR, Fuhlbrigge AL, Marty FM. Non-tuberculous mycobacterial infection among lung transplant recipients: a 15-year cohort study. Transpl Infect Dis. 2012 Oct;14(5):452-460.

${ }^{366}$ Keating MR, Daly JS; AST Infectious Diseases Community of Practice. Nontuberculous mycobacterial infections in solid organ transplantation. Am J Transplant. 2013 Mar; 13 Suppl 4:77-82.

${ }^{367}$ Winthrop KL, Chang E, Yamashita S, lademarco MF, LoBue PA. Nontuberculous mycobacteria infections and anti-tumor necrosis factor- $\alpha$ therapy. Emerg Infect Dis. 2009 Oct;15(10):1556-61.

${ }^{368}$ Chan ED, Iseman MD. Underlying host risk factors for nontuberculous mycobacterial lung disease. Semin Respir Crit Care Med. 2013 Feb;34(1):110-123.

${ }^{369}$ Velayati AA, Farnia P, Mozafari M, MAlekshahlan D, Farahbod AM, Seif S, Rahideh A, Mirsaeidi M. Identification and Genotyping of Mycobacterium tuberculosis isolated from Water and Soil Samples of a Metropolitan City. Chest. 2015 Apr;147(4):1094-102.

370 Thomson R, Tolson C, Carter R, Coulter C, Huygens F, Hargreaves M. Isolation of nontuberculous mycobacteria (NTM) from household water and shower aerosols in patients with pulmonary disease caused by NTM. Microbiol. 2013 Sep;51(9):3006-3011.

${ }^{371}$ Glazer CS, Martyny JW, Lee B, Sanchez TL, Sells TM, Newman LS, Murphy J, Heifets L, Rose CS. Nontuberculous mycobacteria in aerosol droplets and bulk water samples from therapy pools and hot tubs. J Occup Environ Hyg. 2007 Nov; 4(11):831-840.

372 Ashbolt NJ. Environmental (Saprozoic) Pathogens of Engineered Water Systems: Understanding Their Ecology for Risk Assessment and Management. Pathogens. 2015 Jun 19;4(2):390-405.

${ }^{373}$ Lorencova A, Klanicova B, Makovcova J, Slana I, Vojkovska H, Babak V, Pavlik I, Slany M. Nontuberculous mycobacteria in freshwater fish and fish products intended for human consumption. Foodborne Pathog Dis. 2013 Jun;10(6):573-576. 
${ }^{374}$ U.S. Library of Medicine website. Reportable Diseases. Reportable diseases. Jatin M. Vyas, MD, PhD, Assistant Professor in Medicine, Harvard Medical School; Assistant in Medicine, Division of Infectious Disease, Department of Medicine, Massachusetts General Hospital, Boston, MA. Also reviewed by David Zieve, MD, MHA, Isla Ogilvie, PhD, and the A.D.A.M. Editorial team. Updated 5/1/2015. Accessed September 12, 2016.

${ }^{375}$ Centers for Disease Control and Prevention. National Notifiable Diseases Surveillance System (NNDSS). December 17, 2015. Available at: wwwn.cdc.gov/nndss. Accessed September 12, 2016.

${ }^{376}$ U.S. Library of Medicine website. Reportable Diseases. Reportable diseases. Jatin M. Vyas, MD, PhD, Assistant Professor in Medicine, Harvard Medical School; Assistant in Medicine, Division of Infectious Disease, Department of Medicine, Massachusetts General Hospital, Boston, MA. Also reviewed by David Zieve, MD, MHA, Isla Ogilvie, PhD, and the A.D.A.M. Editorial team. Updated 5/1/2015. Accessed September 12, 2016.

377 Sickbert-Bennett EE, Weber DJ, Poole C, MacDonald PDM, and Maillard JM. Completeness of Communicable Disease Reporting, North Carolina, USA, 1995-1997 and 2000-2006. Emerg Infect Dis. 2011 Jan; 17(1):23-29.

378 James L, Roberts R, Jones RC, Watson JT, Hota BN, Kampe LM, Weinstein RA, Gerber SI. Emergency care physicians' knowledge, attitudes, and practices related to surveillance for foodborne disease in the United States. Clin Infect Dis. 2008 Apr 15;46(8):1264-1270.

${ }^{379}$ Blumenthal D. Launching HITECH. N Engl J Med. 2010 Feb 4;362(5):382-385.

${ }^{380}$ Blumenthal D, Tavenner M. The "meaningful use" regulation for electronic health records. N Engl J Med. 2010;363(6):501-504.

381 Overhage JM, Grannis S, McDonald CJ. A comparison of the completeness and timeliness of automated electronic laboratory reporting and spontaneous reporting of notifiable conditions. Am J Public Health. 2008 Feb;98(2):344-350.

${ }^{382}$ World Health Organization website. WHO Report on Global Surveillance of Epidemic-prone Infectious Diseases. Available at who.int. Accessed September 11, 2016.

${ }^{383} 2016$ ICD-10-CM Diagnosis Code A31.9 website. Mycobacterial 
infection, unspecified. Accessed September 15,2016. Available at: http://www.icd10data.com/ICD10CM/Codes/A00-B99/A30-A49/A31-/A31.9

${ }^{384}$ Raju RM, Raju SM, Zhao Y and Rubin EJ. Leveraging Advances in Tuberculosis Diagnosis and Treatment to Address Nontuberculous Mycobacterial Disease. Emerg Infect Dis. 2016 Mar;22(3):365-369 


\title{
APPENDIX
}

\author{
UMIIERSTIY OE \\ LOUISVILLE \\ Subjects Protection Program Office \\ MedCenter One - Suite 200 \\ 501 E. Broadway \\ Louisville, KY 40202-1798 \\ Office: 502.852.5188 Fax: 502.852.2164 \\ DATE: \\ TO: \\ FROM: \\ IRB NUMBER: \\ STUDY TITLE: \\ REFERENCE \# \\ DATE OF REVIEW: \\ IRB STAFF CONTACT: \\ September 02, 2016 \\ Susan O Allen \\ The University of Louisville Institutional Review Board \\ 16.0828 \\ COST OF CARE FOR HOSPITALIZED PATIENTS WITH PULMONARY MYCOBACTERIAL DISEASES \\ IN THE UNITED STATES \\ 617421 \\ 09/01/2016 \\ Barbara Dearinger, BS, CIP \\ (502)852-5987 \\ badear01@louisville.edu \\ The IRB Chair/Vice-Chair has reviewed your submission and the project described does not meet the "Common Rule" \\ definition of human subjects' research. Therefore, this research project does not require IRB review prior to conducting \\ the research. \\ If you have any questions, please contact the IRB analyst listed above or the Human Subjects Protection Program office \\ at hsppofc@louisville.edu. \\ Somcerely, \\ Laura Clark, M.D., Chair \\ Biomedical Institutional Review Board
}




\section{CURRICULUM VITAE}

NAME: $\quad$ Mary Elizabeth Wells Allen

ADDRESS: $\quad 1650$ Sahalee Drive

Lexington, KY 40511

DOB: $\quad$ Corbin, Kentucky - December 22, 1980

\section{EDUCATION}

B.A., Political Science and History

University of Kentucky, Lexington, $\mathrm{KY}$

1999-2002

M.B.A., Entrepreneurship Concentration

University of Louisville, Louisville, KY

2004-2006

AWARDS

Winner Research!Louisville School of Medicine Clinical

Research poster presentation, 2007

First Annual History Department Brent Award, 2002

Summa cum Laude, 2002

University Honors Program, 1999-2002

Arts and Sciences Dean's List, 1999-2002

Merit Scholarship, 2001

Harry S Truman Scholarship Semi-Finalist, 2001

PROFESSIONAL SOCIETIES:

Phi Sigma Pi National Honor Fraternity

Pi Sigma Alpha Political Science Honorary

Phi Alpha Theta History Honorary 
Golden Key International Honour Society

Alpha Lambda Delta Honorary

\section{PUBLICATIONS:}

Mirsaeidi M, Allen MB, Ebrahimi G, Schraufnagel D. Hospital costs in the US for pulmonary mycobacterial diseases. Int J Mycobacteriol. 2015 Sep;4(3):217-221.

Mirsaeidi M, Ebrahimi G, Allen MB, Aliberti S. Pneumococcal vaccine and patients with pulmonary diseases. Am J Med. 2014 Sep;127(9):886.

Mirsaeidi M, Farshidpour M, Allen MB, Ebrahimi G, Falkinham JO. Highlight on advances in nontuberculous mycobacterial disease in North America. Biomed Res Int. 2014;2014:919474.

\section{ABSTRACTS}

Inemesit Umoren, MD, Zehra Palanpurwala, MD, Mary Beth Allen, MBA, Paula Peyrani, MD, Julio Ramirez, MD. Department of Infectious Disease., Narrow Spectrum Antibiotic Therapy is not Associated with Worse Clinical Outcomes in Patients with Hospital-Acquired Pneumonia.

Research!Louisville 2007.

Amir Ali Amjadi, M.D., Ali Asghar Mokras, M.D., Mary Beth Allen, MBA, Paula Peyrani, M.D., Julio Ramirez, M.D. Department of Infectious Disease. Poor Adherence of Physicians with New Guidelines for Short Course Antibiotic Therapy for Hospital-Acquired Pneumonia.

Research!Louisville 2007.

\section{ACKNOLWEDGEMENTS:}

Banoei MM, Mirsaeidi MS, Houshmand M, Payam Tabarsi, Ebrahimi G, Zargari L, Kashani BH, Masjedi MR, Mansouri SD and Ramirez J. Vitamin $D$ receptor homozygote mutant $t t$ and bb are associated with susceptibility to pulmonary tuberculosis in the Iranian population. 2009. Int J Infect Dis.

Mirsaeidi M, Peyrani P, Ramirez J, and the Improving Medicine through Pathway Assessment of Critical Therapy of Hospital-Acquired Pneumonia (IMPACT-HAP) Investigators. Predicting Mortality in Patients with 
Ventilator-Associated Pneumonia: The APACHE II Score versus the New IBMP-10 Score. Clin Infect Dis. 2009 Jul 1;49(1):72-7.

Ramirez J, Aliberti S, Mirsaeidi M, Peyrani P, Filardo G, Amir A, Moffett B, Gordon J, Blasi F, Bordon J. Acute Myocardial Infarction in Hospitalized Patients with Community-Acquired Pneumonia. Clin Infect Dis. 2008 Jul 15;47(2):182-7.

Aliberti S, Myers JA, Peyrani P, Blasi F, Menendez R, Rossi P, Cosentinie $R$, Lopardo G, de Vedia $L$ and Ramirez JA. The role of neutropenia on outcomes of cancer patients with community-acquired pneumonia Eur Respir J 2009; 33: 142-147.

Aliberti S, Amir A, Peyrani P, Mirsaeidi M, Allen M, Moffett BK, Myers J, Shaib F, Cirino M, Bordon J, Blasi F, Ramirez JA. Incidence, etiology, timing, and risk factors for clinical failure in hospitalized patients with community-acquired pneumonia. Chest. 2008 Nov;134(5):955-62. Ramirez J, Aliberti S, Mirsaeidi M, Peyrani P, Filardo G, Amir A, Moffett B, Gordon J, Blasi F, Bordon J. Acute myocardial infarction in hospitalized patients with community-acquired pneumonia. Clin Infect Dis. $2008 \mathrm{Jul}$ $15 ; 47(2): 182-7$.

Mehdi Mirsaeidi, MD, MPH, Fatima Syed, MD, and Elaine S. Jaffe, MDP. Antineutrophil Cytoplasmic AutoantibodyYAssociated Systemic Vasculitis is Associated With Epstein-Barr Virus in the Setting of HIV Infection. Infectious Diseases in Clinical Practice. January 2013;21(1):50-53.

\section{MEETING PRESENTATIONS:}

Hospital costs in the US for pulmonary mycobacterial diseases. The Asian-African Congress of Mycobacteriology, Isfahan, Iran. Poster Presentation, March 2015.

Inemesit Umoren, MD, Zehra Palanpurwala, MD, Mary Beth Allen, MBA, Paula Peyrani, MD, Julio Ramirez, MD. Department of Infectious Disease., Narrow Spectrum Antibiotic Therapy is not Associated with Worse Clinical Outcomes in Patients with Hospital-Acquired Pneumonia. Poster Presentation, Research!Louisville 2007.

Amir Ali Amjadi, MD, Ali Asghar Mokras, MD, Mary Beth Allen, MBA, Paula Peyrani, MD, Julio Ramirez, MD. Department of Infectious Disease. Poor Adherence of Physicians with New Guidelines for Short Course Antibiotic Therapy for Hospital-Acquired Pneumonia. Poster Presentation, Research!Louisville 2007. 


\section{GRANTS}

Sponsor: Federal Government

Ryan White Part C: Early Intervention Services (HRSA; HIVIAIDS Bureau)

Ryan White Part D: Services for Women, Infants, Youth, Children, and their Families (HRSA; HIVIAIDS Bureau)

Sponsor: Industry

CEFTOPED1001: Open-Label, Pharmacokinetic Study of the Penetration of Ceftobiprole into Bone (Ortho-McNeil)

A5951001: Linezolid In The Treatment Of Subjects With Nosocomial Pneumonia Proven To Be Due To Methicillin-Resistant Staphylococcus Aureus (Pfizer)

A Phase 2, Multicenter, Randomized, Double-blind, Comparative Study of the Safety and Efficacy of 2 Doses of Tigecycline Versus Imipenem/Cilastatin for the Treatment of Subjects With HospitalAcquired Pneumonia. (Wyeth)

The SWIFT Study: A Prospective, Randomized, Open-Label Phase IV Study to Evaluate the Rationale of Switching from Fixed-Dose Abacavir (ABC)/Lamivudine (3TC) to Fixed-Dose Tenofovir DF (TDF)/Emtricitabine (FTC) in Virologically-Suppressed, HIV-1 Infected Patients Maintained on a Ritonavir-Boosted Protease InhibitorContaining Antiretroviral Regimen (Gilead)

\section{RESEARCH}

Observational Research

CAPO 202: Prospective, International, Randomized Clinical Trial to Define What Empiric Antibiotic regimen (Moxifloxacin versus Ceftriaxone plus Azithromycin or Clarithromycin) is Associated with Better Outcomes in Hospitalized Patients with Community-Acquired Pneumonia

HAPO 100: Multicenter, Retrospective, Observational Study to Evaluate 
Current Management of Patients with Hospital-Acquired Pneumonia

Sponsored Research

CEFTOPED1001: Open-Label, Pharmacokinetic Study of the Penetration of Ceftobiprole into Bone (Ortho-McNeil)

Persistent Chlamydia Pneumoniae in Human Coronary Arothema (American Heart Association)

A5951001: Linezolid In The Treatment Of Subjects With Nosocomial Pneumonia Proven To Be Due To Methicillin-Resistant Staphylococcus Aureus (Pfizer)

A Phase 2, Multicenter, Randomized, Double-blind, Comparative Study of the Safety and Efficacy of 2 Doses of Tigecycline Versus Imipenem/Cilastatin for the Treatment of Subjects With HospitalAcquired Pneumonia. (Wyeth) 